\title{
Demonstration of a new gamma spectrometer designed for radiometric surveying with an unmanned aircraft system
}

\author{
by \\ Carolyn Chen, B.Sc.E.

\begin{abstract}
A thesis submitted to the
Faculty of Graduate and Postdoctoral Affairs

in partial fulfillment of the requirements for the degree of
\end{abstract}

Master of Science in Earth Sciences

Department of Earth Sciences

Carleton University

Ottawa, Ontario

November, 2018

(C) 2018

Carolyn Chen 


\section{Abstract}

The performance of a radiometric unmanned aircraft system (UAS) composed of a directional gamma spectrometer, the Advanced Radiation Detector for Unmanned Aircraft System Operations (ARDUO), and a medium-lift helicopter airframe was demonstrated. The ARDUO's properties of the full-width half maximum energy resolution, linearity, gain stability, intrinsic photopeak efficiency, sheet source sensitivity and directional response were characterized in laboratory experiments. Smallscale trials were conducted over a single point source and two point sources of ${ }^{137} \mathrm{Cs}$ at a $27 \mathrm{~m}$ separation. The single point source strength was measured at $175 \mathrm{MBq} \pm 39 \mathrm{MBq}$, which is within error of the true source strength of $161 \mathrm{MBq}$. The directional information was shown to improve source locating by differentiating the two point sources. A large-scale 'L'-shaped distributed source trial using ${ }^{140}$ La was completed. The total activity distributed in the area was estimated at $12 \mathrm{GBq} \pm 1 \mathrm{GBq}$. The directional information was shown to improve constraint of the 'L'-shaped distribution area. 


\section{Acknowledgments}

I would first like to thank my supervisors Dr. Laurel Sinclair and Dr. Claire Samson for their support and guidance throughout this project. Dr. Sinclair was a great help in completing the trials with the UAS, taking time out of her busy schedule to help operate the UAS. Dr. Samson ensured that the project was kept on track, and was always patient in her guidance and providing feedback on my work.

I would to offer a sincere thank you those I have worked with at Natural Resources Canada. Richard Fortin has been a great help as a part of the operating crew of the UAS, and for letting me participate in his field trials surveying the Allan Lake carbonatite in Algonquin Park. Dr. Andrew McCann was always there for support an guidance on the data processing methods. I would like to also thank Maurice Coyle for helping operate the UAS, and Jordan Harvey for his help with the laboratory measurements.

I would also like to extend a warm thank you to the team at Defence Research and Development Canada Suffield for their collaboration on the distributed source trial. They were able to create a unique situation where we could survey over a known distributed source of radiation, which has provided some invaluable results for this research project.

Radiation Solutions Inc. has provided excellent support with the ARDUO and their RadAssist software. Thank you for your tireless work to implement changes to the software and to the ARDUO when issues arose. 
Those working at ING Robotic Aviation Inc. have been a great help in troubleshooting difficulties with the Responder UAS. I would like to especially thank Michael Nunan for his incredible support and and his eagerness to help.

Finally, I would like to thank my family and friends. Without their support I would have never made it this far.

This work was supported by Canada's Centre for Security Science as a part of CSSP-2016-TI-2290. 


\section{Statement of Contribution}

I certify that the content of this thesis is my own work.

I presented the results from the direction reconstruction experiments, the two point sources trial and the distributed source trial at the 17th Symposium on Radiation Measurements and Applications [1]. I wrote the paper presenting the same results that has been published in the journal Nuclear Instruments and Methods in Physics Research Section A [2].

Chapter 2 - Instrumentation. The Responder UAS was acquired by NRCan from ING Robotic Aviation Inc. [3]. The ARDUO was designed by Dr. Laurel Sinclair using $\mathrm{CsI}(\mathrm{Tl})$ crystals and silicon photomultipliers from [4]. Radiation Solutions Inc. [5] designed and built the electronics and external housing of the ARDUO, as well as the RadAssist software. I performed initial measurements for each experiment (linearity, energy resolution, gain stability, intrinsic photopeak efficiency and direction reconstruction) to determine the ideal experimental setup and geometry. I collected and analyzed the data for each of the laboratory experiments. Jordan Harvey (Student, NRCan) assisted with part of the direction reconstruction measurements. Dr. Andrew McCann (NRCan) assisted with the intrinsic photopeak efficiency measurements. The sheet source sensitivity simulations were conducted by Dr. Andrew McCann. I wrote $\mathrm{C}++$ codes using the CERN ROOT $\mathrm{C}++$ libraries for analyzing the data from each experiment and for plotting the results. The direction vector reconstruction algorithm was created by Dr. Laurel Sinclair. I implemented the algorithm in my data analysis $\mathrm{C}++$ code. 
Chapter 3 - Methods. The flight planning methods are based on typical aerial surveying methods $[6,7]$. The pre-flight operations regarding the Responder UAS are summarized from the Responder manual provided by ING Aviation Robotics Inc. [8]. I determined the data collection procedures for the ARDUO. The data processing methods are partially based on traditional data processing methods presented in literature $[6,7]$. I developed the methods for: the integration of the autopilot data with the radiation data, the heading correction, the direction vector map overlay display, and the direction vector projection and point density process. I wrote $\mathrm{C}++$ codes using the CERN ROOT $\mathrm{C}++$ libraries for the data analysis and for producing histograms of the results. I used ArcGIS to produce the interpolated maps of the data, as well as to perform the point density analysis using ArcGIS's Kernel Density function [9].

Chapter 4 - Point Source Trials. I planned the flights for the point source trials. Dr. Laurel Sinclair determined the source locations. Dr. Laurel Sinclair (Safety Pilot) and I (GCS Operator) flew the NRCan radiometric UAS. I analyzed and plotted the data using my $\mathrm{C}++$ codes and ArcGIS, following my procedures described in Chapter 3.

Chapter 5 - Distributed Source Trial. Dr. Laurel Sinclair planned the ${ }^{140}$ La source distribution pattern and planned the flights for the distributed source trial. The team at DRDC Suffield (Blake Beckman, Anna Rae Green, Blaine Fairbrother, Dan White) determined the experimental procedures and the procedures for distributing the source [10]. The NRCan radiometric UAS was flown by Richard Fortin (NRCan, Safety Pilot), Maurice Coyle (NRCan, GCS Operator), and I (ARDUO Data Quality Monitor). I analyzed and plotted the data using my $\mathrm{C}++$ codes and ArcGIS, following my procedures described in Chapter 3. 


\section{Table of Contents}

Abstract $\quad$ ii

Acknowledgments $\quad$ iii

Statement of Contribution $\quad$ V

Table of Contents vii

List of Tables $\quad$ x

List of Figures $\quad$ xi

List of Acronyms xiv

List of Symbols $\quad$ xvii

1 Introduction 1

1.1 Applications . . . . . . . . . . . . . . . . . . 1

1.2 Research Objectives. . . . . . . . . . . . . . . 4

1.3 Background .......................... 5

1.3.1 Radiation Theory . . . . . . . . . . . . . . 5

1.3.2 Gamma Spectrometers . . . . . . . . . . . . . 7

1.3.3 Natural Radioactivity . . . . . . . . . . . . . . . . 14

1.3.4 Manned Aerial Radiometric Surveying . . . . . . . . . . . . . 16 
1.3.5 UAS Radiometric Surveying . . . . . . . . . . . . . 18

2 Instrumentation $\quad 20$

2.1 Responder UAS . . . . . . . . . . . . . . . . . . 20

2.1 .1 Airframe . . . . . . . . . . . . . . . . . . . . . . 21

2.1 .2 Ground Control Station . . . . . . . . . . . . . . . . 22

2.1 .3 Telemetry Systems . . . . . . . . . . . . . . . . 25

2.1 .4 Autopilot . . . . . . . . . . . . . . . . . . 28

2.2 Advanced Radiation Detector For Unmanned Aircraft System Operations 30

2.2.1 Self-Shielding Crystal Configuration . . . . . . . . . . . . 32

$2.2 .2 \quad$ Linearity . . . . . . . . . . . . . . . . . . . . . . . 34

2.2.3 Full Width Half Maximum Energy Resolution . . . . . . . . 36

2.2 .4 Gain . . . . . . . . . . . . . . . . . . . . 39

2.2.5 Point Source Intrinsic Photopeak Efficiency _ . . . . . . . 41

2.2.6 Sheet Source Sensitivity _. . . . . . . . . . . . . 46

2.2.7 Direction Reconstruction . . . . . . . . . . . . . . . . 48

3 Methods $\quad 56$

3.1 Data Acquisition Methods . . . . . . . . . . . . . 56

3.1 .1 Flight Planning . . . . . . . . . . . . . . . . 57

3.1 .2 Pre-flight Operations . . . . . . . . . . . . . . . . 62

3.1 .3 In-flight Monitoring . . . . . . . . . . . . . . . . . 64

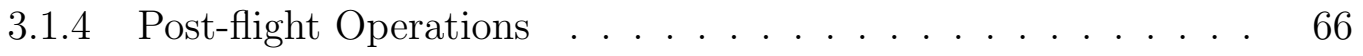

3.2 Data Processing Methods . . . . . . . . . . . . . . 67

3.2 .1 Count Rate Determination . . . . . . . . . . . . . . 67

$3.2 .2 \quad$ Background Subtraction $\ldots \ldots \ldots$. . . . . . . . 68

3.2 .3 Lag Correction . . . . . . . . . . . . . . . . . 69

3.2.4 Back-decay Correction . . . . . . . . . . . . . . . . . 69 
3.2.5 Radiation and Autopilot Data Integration . . . . . . . . . . 71

3.2.6 Altitude Leveling . . . . . . . . . . . . . . . . . 71

3.2.7 Photopeak Count Rate Mapping . . . . . . . . . . . . . . . 74

3.2.8 Direction Reconstruction . . . . . . . . . . . . . . . . 74

3.2.9 Direction Vector Projection _. . . . . . . . . . . . . 76

3.2.10 Source Strength Estimation _. . . . . . . . . . . . . 77

4 Point Source Trials $\quad 81$

4.1 Experimental Setup . . . . . . . . . . . . . . . . 81

4.2 Results.......................... 83

4.2 .1 One ${ }^{137}$ Cs Source Survey . . . . . . . . . . . . . . . . . . . 87

4.2.2 Two ${ }^{137}$ Cs Sources Survey . . . . . . . . . . . . . . . . . . . 94

4.3 Discussion . . . . . . . . . . . . . . . . . . . . . . . . . . . . . . 98

5 Distributed Source Trial 102

5.1 Experimental Setup . . . . . . . . . . . . . . . . 102

5.2 Results . . . . . . . . . . . . . . . . . 107

5.3 Discussion . . . . . . . . . . . . . . . . . . . . . . 118

$6 \quad$ Summary and Conclusions $\quad 123$

$\begin{array}{ll}\text { References } & 128\end{array}$ 


\section{List of Tables}

2.1 List of isotopes and their associated photopeak energies that were used for the linearity and energy resolution tests. . . . . . . . . . . . . 35

2.2 Proportionality constants for each crystal of the ARDUO calculated from a fit of the FWHM energy resolution versus true energy plot. . . 38

2.3 Intrinsic photopeak efficiency measurements. . . . . . . . . . . . 45

2.4 Sensitivity function constants. . . . . . . . . . . . . . . 47

4.1 Point source locations in UTM Zone 18. . . . . . . . . . . . . . 82

4.2 Summary of the flights conducted for the point source trials. . . . . . 83

4.3 Source strength estimation values. . . . . . . . . . . . . . . 94 


\section{List of Figures}

1.1 The linear attenuation coefficient for $\mathrm{NaI}$ as a function of photon energy. 9

1.2 Example spectrum from a ${ }^{137} \mathrm{Cs}$ source. . . . . . . . . . . . . . 10

1.3 Typical spectrum from a manned aerial radiometric survey. . . . . . . 15

1.4 Radiometric map of the Allan Lake carbonatite glacial train anomaly in Ontario. . . . . . . . . . . . . . . . . . . 17

2.1 The Responder airframe with the ARDUO mounted beneath the main rotor. . . . . . . . . . . . . . . . . . . 23

2.2 The Ground Control Station. . . . . . . . . . . . . . . . 26

2.3 The radio control transmitter. . . . . . . . . . . . . . . 27

2.4 The Pixhawk 1 autopilot. . . . . . . . . . . . . . . . . . . 29

2.5 The ARDUO. . . . . . . . . . . . . . . . . . . 33

2.6 Real-time data view during an aerial survey. . . . . . . . . . . . . . 34

2.7 Spectrum collected from crystal 1 of the ARDUO using a ${ }^{152}$ Eu check source. . . . . . . . . . . . . . . . 36

2.8 Linearity of the ARDUO. . . . . . . . . . . . . . . 37

2.9 The measured FWHM energy resolution for each crystal of the ARDUO when calibrated at $0.662 \mathrm{MeV}$. . . . . . . . . . . . . . . 39

2.10 The mean photopeak energy of each crystal of the ARDUO and the internal temperature of the ARDUO over time. . . . . . . . . . . . . 42

2.11 Experimental setup for the intrinsic photopeak efficiency measurements. 43 
2.12 Plot of the measured photopeak count rates and intrinsic photopeak efficiencies as a function of distance from the center of the ARDUO. . 46

2.13 Simulated sensitivity to a sheet source as a function of the altitude of the ARDUO for a $1.596 \mathrm{MeV}$ gamma ray source. . . . . . . . . . . . 48

2.14 Laboratory experiment setup with the ARDUO, Responder and wooden frame. . . . . . . . . . . . . . . . . . . 50

2.15 The experimental setup for the UAS for direction reconstruction measurements at larger distances. . . . . . . . . . . . . . . . 51

2.16 Results from the laboratory direction reconstruction tests. . . . . . . 55

3.1 Mission plan created using the Mission Planner software [11] for the distributed source trial. . . . . . . . . . . . . . . . . 58

3.2 The Mission Planner software real-time display during the flight over the distributed source. . . . . . . . . . . . . . . . 65

3.3 Data processing flowchart. . . . . . . . . . . . . 68

3.4 Lag correction. . . . . . . . . . . . . . . . . 70

3.5 Visualization of the process of projecting the direction vectors collected during flight down to a ground surface. . . . . . . . . . . . . . . . . 77

4.1 Survey area in the compound of the NRCan Geomagnetic Laboratory. 84

4.2 Background survey for the point source trials. . . . . . . . . . . . 86

4.3 Representative $1 \mathrm{~s}$ energy spectra for a data point located over the ${ }^{137} \mathrm{Cs}$ source for the survey over one point source of ${ }^{137} \mathrm{Cs} . . . . . . \quad 88$

4.4 Representative average survey energy spectra from crystal 1 of the ARDUO for the single ${ }^{137} \mathrm{Cs}$ point source survey. . . . . . . . . . . . . 89

4.5 Photopeak count rate map from a survey over a single point source of ${ }^{137}$ Cs. . . . . . . . . . . . . . . . . . . . . 91

4.6 Projection map of the survey over a single point source. . . . . . . . . 92 
4.7 Histogram of the altitude measurements for the single ${ }^{137} \mathrm{Cs}$ point source survey. . . . . . . . . . . . . . . . . . . . . . . 94

4.8 Photopeak count rate map from a survey over two point sources of ${ }^{137}$ Cs. 96

4.9 Projection map of the survey over two point sources. . . . . . . . . 97

5.1 The UGVs used in the Suffield distributed source trial. . . . . . . . . 103

5.2 Map of the distributed source trial area. . . . . . . . . . . . . 106

5.3 Background ${ }^{140} \mathrm{La} 1.596 \mathrm{MeV}$ photopeak map of the Suffield distributed source survey area. . . . . . . . . . . . . . . . . . . . 108

5.4 Representative $1 \mathrm{~s}$ energy spectra for a data point located over the ${ }^{140} \mathrm{La}$ source for the distributed source survey. . . . . . . . . . . . . . 110

5.5 Energy spectra from crystal 1 of the ARDUO while flying over the

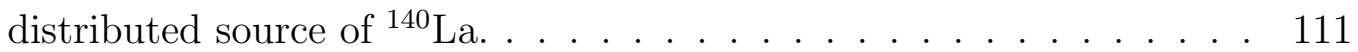

5.6 Back-decayed photopeak count rate map of the ' $\mathrm{L}$ '-shaped distribution of ${ }^{140}$ La. . . . . . . . . . . . . . . . . . . . . . . . . 113

5.7 Histogram of the altitude measurements for the distributed source trial. 114

5.8 Surface activity map of the 'L'-shaped distribution of ${ }^{140} \mathrm{La} . \quad$. . . . . 117

5.9 Projection map of the 'L'-shaped distribution of ${ }^{140} \mathrm{La} . \quad$. . . . . . . . 119 


\section{List of Acronyms}

\begin{tabular}{ll}
\multicolumn{1}{c}{ Acronym } & Meaning \\
\hline${ }^{40} \mathrm{Ar}$ & Argon-40 \\
ARDUO & Advanced Radiation Detector for \\
& Unmanned Aircraft System Operations \\
${ }^{133} \mathrm{Ba}$ & Barium-133 \\
${ }^{214} \mathrm{Bi}$ & Bismuth-214 \\
$\mathrm{Bq}$, & Becquerel, disintegrations per second, \\
$\mathrm{kBq}, \mathrm{MBq}, \mathrm{GBq}$ & kilo-becquerel, mega-becquerel, giga-becquerel \\
$\mathrm{Ci}$ & Curie \\
${ }^{60} \mathrm{Co}$ & Co-60 \\
$\mathrm{cps}$ & Counts per second \\
${ }^{137} \mathrm{Cs}$ & Cesium-137 \\
$\mathrm{CsI}, \mathrm{CsI}(\mathrm{Tl})$ & Cesium iodide, thallium-doped cesium iodide \\
$\mathrm{CZT}$ & Cadmium zinc telluride \\
$\mathrm{EDT}$ & Eastern day time \\
$\mathrm{EKF}$ & Extended Kalman Filter
\end{tabular}




\begin{tabular}{|c|c|}
\hline Acronym & Meaning \\
\hline${ }^{152} \mathrm{Eu}$ & Europium-152 \\
\hline $\mathrm{eV}, \mathrm{keV}, \mathrm{MeV}$ & Electron volt, kilo-electron volt, mega-electron volt \\
\hline FWHM & Full-width half maximum \\
\hline $\mathrm{g}, \mathrm{kg}$ & Gram, kilogram \\
\hline GCS & Ground Control Station \\
\hline $\mathrm{GHz}$ & Gigahertz \\
\hline GNSS & Global Navigation Satellite System \\
\hline GPS & Global Positioning System \\
\hline h & Hour \\
\hline hdop & Horizontal dilution of precision \\
\hline IDW & Inverse distance weighted \\
\hline${ }^{40} \mathrm{~K}$ & Potassium-40 \\
\hline $\mathrm{kPa}$ & Kilo-Pascal \\
\hline $\mathrm{kt}$ & Knot \\
\hline $\mathrm{L}$ & Litre \\
\hline${ }^{140} \mathrm{La}$ & Lanthanum-140 \\
\hline $\mathrm{m}, \mathrm{km}, \mathrm{cm}$ & Metre, kilometre, centimetre \\
\hline MDT & Mountain day time \\
\hline
\end{tabular}




\begin{tabular}{|c|c|}
\hline Acronym & Meaning \\
\hline $\min$ & Minute \\
\hline${ }^{54} \mathrm{Mn}$ & Magnesium-54 \\
\hline $\mathrm{NaI}, \mathrm{NaI}(\mathrm{Tl})$ & Sodium iodide, thallium-doped sodium iodide \\
\hline NRCan & Natural Resources Canada \\
\hline RSI & Radiation Solutions Inc. [5] \\
\hline RTL & Return to Launch \\
\hline $\mathrm{s}, \mu \mathrm{s}$ & Second, microsecond \\
\hline $\mathrm{Sv}$ & Sievert \\
\hline${ }^{232} \mathrm{Th}$ & Thorium-235 \\
\hline${ }^{208} \mathrm{Tl}$ & Thallium-208 \\
\hline${ }^{238} \mathrm{U}$ & Uranium-238 \\
\hline UAS & Unmanned aircraft system \\
\hline $\mathrm{UGV}$ & Unmanned ground vehicle \\
\hline UTC & Coordinated universal time \\
\hline
\end{tabular}




\section{List of Symbols}

\begin{tabular}{ll} 
Symbol & Meaning \\
\hline$a$ & Fit constant \\
$b$ & Fit constant \\
$c$ & Fit constant \\
$A$ & Source strength \\
$A_{\mathrm{S}, i}$ & Surface activity concentration for data point $i$ \\
$\frac{\mathrm{d} N}{\mathrm{~d} t}$ & Activity, or decay rate \\
$A_{\mathrm{ARDUO}}$ & Cross-sectional area of the ARDUO \\
$\epsilon_{\mathrm{int}}(E)$ & Intrinsic photopeak efficiency at energy $E$ \\
$E_{\mathrm{True}}$ & Branching ratio \\
$E_{\mathrm{h}}$ & Count rate altitude correction factor \\
& Energy photopeak energy
\end{tabular}




\begin{tabular}{|c|c|}
\hline Symbol & Meaning \\
\hline$h$ & Altitude above ground level \\
\hline$h_{0}$ & Nominal survey altitude above ground level \\
\hline$h_{i}$ & Altitude above ground level for data point $i$ \\
\hline$i$ & Data point, dataset, ARDUO crystal number \\
\hline$K$ & Proportionality constant \\
\hline$\lambda$ & Decay constant \\
\hline$\mu$ & Linear attenuation coefficient \\
\hline$\mu_{\text {air }}(E)$ & Linear attenuation coefficient for air at energy $E$ \\
\hline$\mu_{\mathrm{h}}$ & Energy window height attenuation coefficient \\
\hline$N$ & Number of nuclei \\
\hline$N_{0}$ & $\begin{array}{l}\text { Number of gamma rays incident on a material, or } \\
\text { spectrometer }\end{array}$ \\
\hline$N_{\mathrm{A}}$ & $\begin{array}{l}\text { Number of gamma rays attenuated by a material, or } \\
\text { spectrometer }\end{array}$ \\
\hline$N_{\mathrm{x}}$ & Number of gamma rays transmitted through a material \\
\hline$N_{\text {dataset }}$ & Number of datasets \\
\hline$n$ & Measured count rate \\
\hline$n_{i}$ & Measured count rate for data point $i$ \\
\hline$n_{\mathrm{c}, i}$ & Back-decayed count rate for data point $i$ \\
\hline$n_{\mathrm{p}}$ & Measured photopeak count rate \\
\hline
\end{tabular}




\begin{tabular}{|c|c|}
\hline Symbol & Meaning \\
\hline$n_{\mathrm{p}, i}$ & Photopeak count rate for data point $i$ \\
\hline$n_{\mathrm{h}}$ & Altitude corrected count rate \\
\hline$n_{\mathrm{crys}, i}$ & $\begin{array}{l}\text { Count rate in the ARDUO crystal number } i \text {, where } i \text { is } \\
\text { from } 1 \text { to } 8\end{array}$ \\
\hline$\Phi$ & Theoretical photopeak count rate \\
\hline$R$ & $\begin{array}{l}\text { Distance from the radioactive source to the center of } \\
\text { a spectrometer }\end{array}$ \\
\hline$R_{\mathrm{FWHM}}(E)$ & Full-width half maximum energy resolution for energy $E$ \\
\hline RMSD & Root mean squared deviation \\
\hline$S$ & Sheet source sensitivity \\
\hline$S_{i}$ & Sheet source sensitivity at data $i$ \\
\hline$\sigma$ & Standard deviation \\
\hline$t_{1 / 2}$ & Half-life \\
\hline$t_{0}$ & Reference time of 8:00 MDT on March 20th, 2018 \\
\hline$t_{i}$ & Time for data point $i$ \\
\hline$x$ & Thickness \\
\hline $\bar{x}$ & Mean azimuth (elevation) angle for all datasets \\
\hline$x_{i}$ & Azimuth (elevation) angle for dataset $i$ \\
\hline $\mathbf{v}$ & Direction vector \\
\hline$u$ & Horizontal component of the direction vector \\
\hline
\end{tabular}




\section{Symbol Meaning}

$v$

$w$
Horizontal component of the direction vector perpendicular to $u$

Vertical component of the direction vector 


\section{Chapter 1}

\section{Introduction}

This chapter presents the applications for this research thesis, the research objectives, and background information. The background information in Section 1.3 covers radiation theory, gamma ray detection and a review of radiometric surveying methods with reference to ongoing work in the literature.

\subsection{Applications}

Radiometric surveying is a geophysical method that uses spectrometers that measure the gamma radiation produced by natural and anthropogenic radioactive sources. Surveys can be conducted with ground-based spectrometers, ranging in size from the smaller hand-held spectrometers, to larger car- or truck-borne systems. Surveys can also be conducted with aerial gamma spectrometer systems that are mounted on manned fixed-wing or helicopter aircraft, and more recently are integrated with unmanned aircraft systems (UASs) [12-21]. The data collected are used to produce radiation maps that show the spatial distribution of the radiation present within the survey area. Radiometric surveying is used in a variety of applications that are discussed in the following paragraphs.

One of the main applications for radiometric surveys is for mineral exploration. Radiometric surveying is conducted to estimate the near-surface concentrations of 
the naturally-occurring radioactive elements uranium-238 $\left({ }^{238} \mathrm{U}\right)$, thorium-235 $\left({ }^{232} \mathrm{Th}\right)$ and potassium-40 $\left({ }^{40} \mathrm{~K}\right)$. Gamma radiation has a high attenuation rate in rocks and soil, therefore only radiation being produced within the top $50 \mathrm{~cm}$ of the Earth's surface is being measured [22]. The concentration estimates for each of the three isotopes are used to produce maps to interpret the lithologies or soil types in the area. The interpretations can lead to mineral deposit discoveries, as anomalously high concentrations of ${ }^{238} \mathrm{U}$ and ${ }^{232} \mathrm{Th}$ can directly indicate the presence of uranium or thorium deposits. High concentrations of ${ }^{40} \mathrm{~K}$ and low $\mathrm{Th} / \mathrm{K}$ ratios can indicate the presence of potassic alteration which is associated with hydrothermal deposits, volcanic massive sulphide deposits, and epithermal deposits [7, 22]. Low concentrations of ${ }^{40} \mathrm{~K}$ and ${ }^{238} \mathrm{U}$ with high concentration haloes have also been indicative of surficial microseepage or electrochemical cells, which are often present above petroleum deposits [7].

Radiometric surveys are also used in environmental monitoring around functioning and decommissioned nuclear power plants, nuclear waste facilities, and uranium mining facilities [21]. These surveys monitor the concentrations of man-made radioactive material such as cesium-137 $\left({ }^{137} \mathrm{Cs}\right)$ and cobalt-60 $\left({ }^{60} \mathrm{Co}\right)$. The survey maps produced can be used to locate hazardous zones of contamination, and guide remediation efforts. Environmental radiometric surveys are often conducted to mitigate the risk to nuclear facility workers and the public. Surveys are often conducted periodically to locate areas of new contamination, monitor the progress of remediation, and to track the movement of contamination.

Furthermore, radiometric surveys are a part of the response efforts to radiological and nuclear events. Two major events have occurred in recent history, the Chernobyl Nuclear Reactor accident in 1986, and the Fukushima Dai-ichi Power Plant accident in 2011. Radiometric surveying is an important aspect of the initial response efforts, as it produces maps of the distribution and quantities of the radioactive fallout $[6,23]$. This information is used to plan evacuations, and guide incident management strategies to 
prevent further harm to the public and nuclear facility workers. Follow-up surveys are conducted in the time after the initial stages of an incident to monitor remediation and migration of the fallout.

Natural Resources Canada (NRCan) is committed to support increased mineral exploration efforts to inform responsible resource development. This includes the collection of new field data, as well as applying new airborne geophysical techniques. NRCan is also responsible for conducting radiometric surveys in response to radiological and nuclear incidents. To support these two mandates, NRCan requires high sensitivity systems that are easily deployable in order to detect, locate and identify radioactive sources. For these reasons, NRCan is investigating the use of UASs for radiometric surveying.

The use of a UAS can provide several benefits to radiometric surveying. They are capable of producing higher spatial resolution radiometric maps when compared to the maps produced using a manned aircraft. The spatial resolution of a map is limited by the survey platform's speed and altitude. A UAS is capable of flying lower and slower than a manned aircraft which can improve the spatial resolution of the radiometric map. Ground-based surveys can also produce high resolution maps, but they can be arduous as the operators may need to carry the equipment over large distances, and they can be hindered by difficult terrain. Another benefit is the quick deployment, and operational flexibility of a UAS. For manned aircraft, an airport is required for takeoff and landing, which can be at a large distance from the survey location. They must also adhere to a strict set of regulations that can increase the time taken prior to deployment. A UAS does not require an airport for operations, and can therefore be deployed in remote areas closer to survey areas and avoid conflicts with the increased amounts of air traffic around airports. In nuclear response situations, a UAS is also able to provide an additional level of safety for operators as a UAS can be operated at a safe distance from hazardous areas. 
NRCan has developed a novel gamma spectrometer called the Advanced Radiation Detector for UAS Operations (ARDUO) that has been integrated with the Responder main- and tail-rotor medium-lift UAS built by ING Robotic Aviation Inc. [3]. The remainder of this chapter includes the research objectives, background information on radiation theory and gamma spectrometry, and a review of current manned and unmanned radiometric systems. Chapter 2 contains a detailed description of the ARDUO and Responder systems. Chapter 3 describes the UAS data acquisition and data processing methods. Chapter 4 discusses the point source trials that were conducted to examine the UAS's small-scale surveying capabilities. Chapter 5 discusses the distributed source trial that was conducted to determine the UAS's large-scale surveying capabilities. The thesis is concluded in Chapter 6 with a summary and recommendations for future work.

The results from the direction reconstruction experiments, the two point sources trial and the distributed source trial have been presented at the 17th Symposium on Radiation Measurements and Applications [1], and have been published in the peerreviewed journal Nuclear Instruments and Methods in Physics Research Section A [2].

\subsection{Research Objectives}

This thesis focuses on the performance of the ARDUO and Responder UAS for radiometric surveying. Prior to being able to use the system for geophysical exploration or nuclear emergency response, the ARDUO requires characterization under laboratory

conditions. Tests surveys are also required to determine the system's response to known sources. These tests will provide the necessary system response characteristics for mapping unknown geological features or material of security concern.

The main objectives of the research are listed below: 
1. Characterize the ARDUO's linearity, energy resolution, gain stability, and direction reconstruction capability under controlled laboratory conditions.

2. Demonstrate the survey capabilities of the UAS in mapping point sources and distributed sources.

3. Demonstrate the use of the direction information from the ARDUO to improve surveying results for point sources and distributed sources.

\subsection{Background}

This section outlines the principles of radiation theory and gamma spectrometry, as well as how they are used in aerial radiometric surveying. A brief literature review of current UAS radiometric systems and their capabilities is included.

\subsubsection{Radiation Theory}

Chemical elements are defined by the number of protons that are present within the nucleus of the atom. Isotopes of an element have a different mass due to a difference in the number of neutrons within the nucleus. An isotope can be unstable, where the nucleus has a surplus of energy. To reduce the surplus energy and become more stable, the isotope releases radiation in the form of particles or energy. The rate at which the isotope's nuclei decay is defined by the activity,

$$
\frac{\mathrm{d} N}{\mathrm{~d} t}=-\lambda N,
$$

where $N$ is the number of nuclei and $\lambda$ is the decay constant $\left(\mathrm{s}^{-1}\right)$. The standard unit for activity is the Becquerel (Bq), the number of disintegrations per second. Another commonly used unit is the Curie $(\mathrm{Ci})$, where $1 \mathrm{Ci}$ is equal to $3.7 \times 10^{10} \mathrm{~Bq}$ [24]. The $\mathrm{Ci}$ is still used as it corresponds approximately to the decay rate of $1 \mathrm{~g}$ of radium and 
is therefore a more intuitive quantity than the Bq. The decay constant, $\lambda\left(\mathrm{s}^{-1}\right)$, is defined by the half-life, $t_{1 / 2}(\mathrm{~s})$, of the isotope by,

$$
\lambda=\frac{\ln (2)}{t_{1 / 2}} .
$$

The half-life of the isotope, $t_{1 / 2}$, is the experimentally determined time required for the isotope to decay to half its initial amount.

Radioactive isotopes can decay in a series of steps prior to reaching a stable form. The sequence is called a decay chain, where each step produces some amount of radiation and one or more isotopes called daughter products. The radiation produced at each decay step takes the form of an alpha particle, beta particle or gamma ray, and therefore defines the decay step as an alpha, beta, or gamma decay [24].

Alpha radiation is a particle composed of two protons and two neutrons. Beta radiation is a particle identical to an electron or a positron. These two types of radiation attenuate over a short distance, therefore they cannot be used for aerial radiometric surveying. Gamma radiation is produced when a daughter product of an alpha or beta decay is left in an excited state. The daughter product relaxes, and releases the extra energy in the form of a gamma ray. Gamma radiation is used for aerial radiometric surveying as it only consists of electromagnetic energy, which has a significantly lower attenuation rate than alpha and beta radiation.

A decay step can also produce more than one daughter product, where the probability of producing each daughter product is defined by the branching ratio, BR. Each decay step also has an associated half-life, where some decay steps may last seconds and others thousands of years [22].

Each radioactive isotope produces a characteristic set of gamma rays at known energies as it decays. The energy of each gamma ray is measured in electron volts $(\mathrm{eV})$, defined as the kinetic energy gained by an electron from its acceleration through 
a potential difference of $1 \mathrm{~V}$ [24]. Gamma ray energies are commonly measured in kilo electron volts $(\mathrm{keV})$ and mega electron volts $(\mathrm{MeV})$.

\subsubsection{Gamma Spectrometers}

\section{Theory}

Gamma spectrometers are instruments that are made of a material that will interact with an incident gamma ray and produce a measurable signal. The amount of gamma rays that interact with the material within a spectrometer is dependent on the linear attenuation coefficient of the material, $\mu\left(\mathrm{m}^{-1}\right)$. Using the linear attenuation coefficient for a given material of thickness $x(\mathrm{~m})$, the number of gamma rays that are transmitted through the material, $N_{\mathrm{x}}$, is defined by,

$$
N_{\mathrm{x}}=N_{0} e^{-\mu x},
$$

where $N_{0}$ is the incident number of gamma rays. Materials with higher atomic numbers and higher densities will have higher $\mu$ values and are therefore more attenuating. The $\mu$ value for each material also varies depending on the gamma ray energy.

Figure 1.1 shows the linear attenuation coefficient as a function of photon, or gamma ray, energy for sodium iodide (NaI) [25], a typical scintillation material. The response would be similar for other scintillating materials. When a gamma ray interacts with matter, there are three mechanisms that can occur. These are the photoelectric effect, Compton scattering and pair-production. The contribution of each interaction mechanism to the absorbed signal is shown using the individually labeled lines. The total attenuation coefficient line represents the sum of all the interaction mechanism lines. This total coefficient line shows that lower gamma ray energies are more readily absorbed by the material than higher energy gamma rays. 
Photoelectric absorption occurs when the gamma ray is completely absorbed by the interaction with the atom. The atom then releases a photoelectron of a characteristic energy that is dependent on the absorbed gamma ray's energy. This effect is the primary interaction mechanism for low energy gamma rays, less than approximately $0.2 \mathrm{MeV}$, as shown in Figure 1.1. Compton scattering is the dominant mechanism for medium energy gamma rays (approximately 0.2 to $7 \mathrm{MeV}$ ). Compton scattering occurs when the incoming gamma ray is deflected by an electron. The gamma ray transfers part of its energy to an electron in the outer shell of the atom. The gamma ray continues to move through the material at a lower energy. The electron is ejected from the from the outer shell. For incoming gamma rays with energies exceeding 1.02 $\mathrm{MeV}$, pair production becomes possible, and is the dominant mechanism for high energy gamma rays (greater than approximately $7 \mathrm{MeV}$ ) [24]. This mechanism occurs when the incoming gamma ray disappears, and produces an electron-positron pair. The positron will quickly be annihilated by any available electron, producing two annihilation photons with energies of $0.511 \mathrm{MeV}$. Fast electrons and positrons also produce bremsstrahlung radiation. When a fast electron moves through matter, a portion of its energy is converted into electromagnetic energy called bremsstrahlung radiation. This radiation can be measured with a gamma spectrometer, and can contribute to the background signal. Gamma rays can also pass through the spectrometer without any interaction, and therefore not be detected at all.

The measured interactions within the spectrometer are summed in a histogram over a period of time to produce an energy spectrum. Often the energy spectrum is displayed as a count rate versus gamma ray energy. The count rate units are typically measured in counts per second (cps). While the count rate unit is similar to the unit for activity, $\mathrm{Bq}$, a $\mathrm{Bq}$ is specific to a disintegration per second. The activity can therefore differ from the measured count rate due to the branching ratio of the isotope, where only a percentage of the disintegrations result in a measurable gamma 


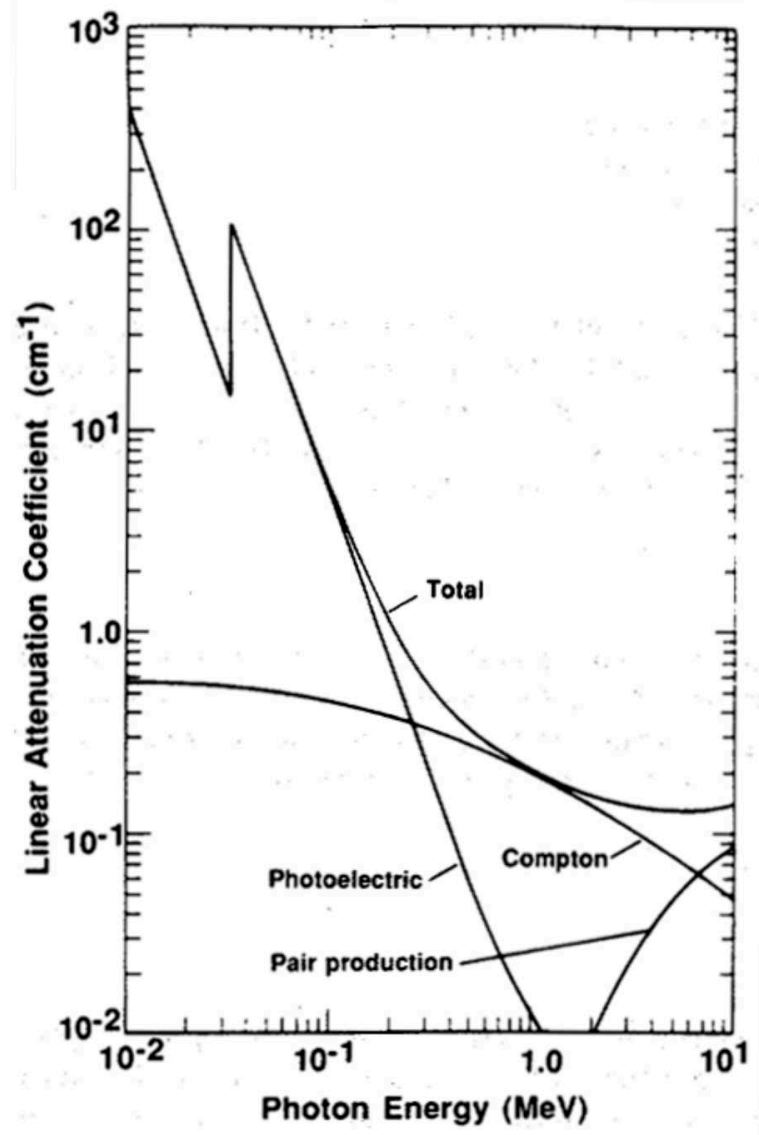

Figure 1.1: The linear attenuation coefficient for $\mathrm{NaI}$ as a function of photon energy. The contributions to the attenuation coefficient from each interaction method, photoelectric absorption, Compton scattering, pair production are shown. The total attenuation coefficient line is the sum of each of the interactions. Figure from Nelson and Reilly [25].

ray emission. To convert the measured count rate into a source activity, assuming the spectrometer measures all emitted gamma rays, the count rate would be divided by the branching ratio.

An energy spectrum contains distinctive features from the series of interactions within the spectrometer. An example spectrum from a ${ }^{137} \mathrm{Cs}$ source is shown in Figure 1.2 to illustrate some of these common features. The decay of ${ }^{137} \mathrm{Cs}$ produces a gamma ray with an energy of $0.662 \mathrm{MeV}$. The resulting spectral feature from this emitted gamma ray energy is a high count rate peak, called a photopeak. The photopeak from the $0.662 \mathrm{MeV}$ gamma emission from ${ }^{137} \mathrm{Cs}$ is shown in the example 
spectrum. The width of the photopeak is a result of fluctuations in the light output from the scintillator and fluctuations in the response of the photomultiplier. This width is defined as the energy resolution and is discussed further in Section 1.3.2. The effects from Compton scattering are shown by the continuous count rate distribution over low energies. The Compton edge is shown, indicating the highest energy that can be deposited in a Compton scatter. A backscatter peak is also shown. This is the result of Compton scattering in the material around and within the spectrometer. Finally, an x-ray is shown. While a gamma spectrometer can measure x-rays, $\mathrm{x}$-rays are not typically used for aerial radiometric surveying. The x-rays are often below the low-energy threshold that is set for the spectrometer to remove the effects from possible low energy noise caused by the spectrometer's electronics.

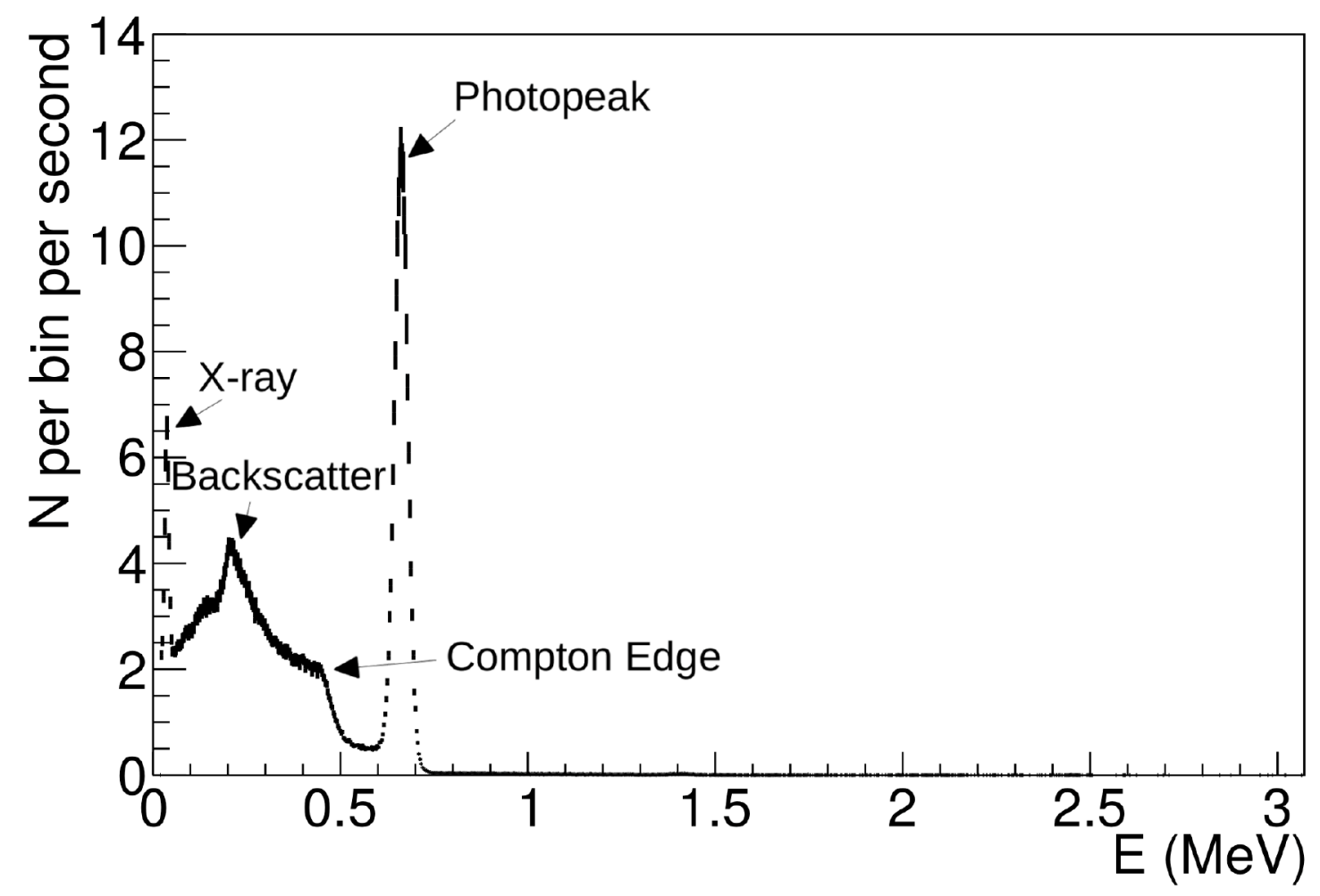

Figure 1.2: Example spectrum from a ${ }^{137} \mathrm{Cs}$ source. The number of counts, N, per histogram bin per second is shown on the y-axis. The energy, $\mathrm{E}$, is shown on the $\mathrm{x}$-axis. Characteristic features features include the $0.032 \mathrm{MeV}$ x-ray, a backscatter peak, the Compton edge, and the $0.662 \mathrm{MeV}$ photopeak. 


\section{Types of Spectrometers}

Spectrometers vary in their composition and methods of gamma ray detection. Often high density materials are used as they have an higher attenuation of gamma rays, and therefore have a higher probability of measuring an incident gamma ray. Using the linear attenuation coefficient for the spectrometer material, $\mu$, the number of gamma rays attenuated by the spectrometer, $N_{\mathrm{A}}$, relative to the number of gamma rays incident on the spectrometer, $N_{0}$, can be defined by,

$$
N_{\mathrm{A}}=N_{0}\left(1-e^{-\mu x}\right) .
$$

A higher linear attenuation coefficient allows the material to absorb more gamma rays, and is therefore able to detect more gamma rays. The number of attenuated gamma rays is also dependent on the thickness of the detection material. As gamma rays can be detected in three-dimensions, the volume of the detection material in the spectrometer therefore affects the spectrometer's detection ability. A larger spectrometer is more effective in detecting and locating radiation, but the larger spectrometer would be heavier, and less maneuverable in an operational context. The detection ability of a spectrometer can be defined by the intrinsic photopeak efficiency, or by the sensitivity. These properties determine how long it takes to collect a statistically significant energy spectrum, as well as the distance to which it is possible to detect a source of a given activity. They also allow for calculations of an unknown source strength. Both of these properties are discussed further in Section 1.3.2.

Semiconductor spectrometers use a solid state semi-conductive material, such as high purity germanium. When a gamma ray interacts with the material, a charge is produced and measured. The measured current is proportional to the incident gamma ray energy [24]. These spectrometers have the best energy resolution, but have traditionally been limited to laboratory use due to the need to cool the high 
purity germanium. A newer material, cadmium zinc telluride (CZT), is now available for field operations. The use of a CZT spectrometer is limited as the material is expensive to produce. CZT spectrometers therefore have small volumes and are less sensitive to gamma radiation.

Scintillator spectrometers use crystals that produce optical photons of characteristic energies when hit with an incident gamma ray. Commonly used scintillators are thallium-doped sodium iodide $(\mathrm{NaI}(\mathrm{Tl}))$ and thallium-doped cesium iodide $(\mathrm{CsI}(\mathrm{Tl}))$. The amount of optical photons produced when the scintillating material is hit with a gamma ray is proportional to the deposited energy of the gamma ray. The optical photons are collected and measured. The signal is then multiplied in a photomultiplier. The signal pulses are sorted into bins according to their energy by an analog to digital converter. The bins are then converted to gamma-ray energies using a calibration parameter. While scintillator spectrometers have a lower energy resolution when compared with semiconductor spectrometers, they are cheaper to produce. Large volume scintillator spectrometers can therefore be produced with high sensitivities. These spectrometers have been widely used for aerial radiometric surveying for their high sensitivities as this allows for the detection of low activity radiation sources at typical survey altitudes of approximately $120 \mathrm{~m}[6]$.

\section{Gamma Spectrometer Properties}

While there are different types of gamma spectrometers, there are general properties that apply to all spectrometer types. These include the energy resolution, efficiency, linearity, gain calibration and dead time [24].

It is possible to calibrate the energy distribution of the energy spectrum by examining how the spectrometer responds to a monoenergetic source. An example spectrum from a monoenergetic source was shown in Figure 1.2. A perfect spectrometer would produce a spectrum with a single line at the source energy of $0.662 \mathrm{MeV}$, 
with a Compton scattering continuum at low energies. As there are a series of fluctuations within the spectrometer for the various steps in the measurement process, the detected energy from the source is smeared across an energy range centered at the source energy. This is defined as the photopeak in the energy spectrum, and it follows a Gaussian distribution. The width of the photopeak is a property that is primarily dependent on the material used in the spectrometer. This width is defined as the energy resolution. The energy resolution is calculated using the full width of the photopeak at half of the maximum energy of the photopeak. The full-width half maximum (FWHM) is then divided by the mean energy of the photopeak, and stated as a percentage. FWHM energy resolutions for scintillator spectrometers used in aerial surveying are typically $8 \%$ at an energy of $0.662 \mathrm{MeV}$.

The intrinsic peak efficiency of a spectrometer is defined as the ratio between the measured number of energy deposits within a photopeak to the number of photopeak gamma rays incident on the spectrometer. The intrinsic peak efficiency determines the amount of radiation that passes through the spectrometer and avoids detection, or undergoes Compton scattering and is therefore not included in the photopeak. Spectrometers are often more efficient at lower incident gamma ray energies as they absorb a larger portion of the incident gamma rays through photoelectric absorption. At higher energies, the gamma rays are more likely to pass through the spectrometer or undergo Compton scattering, therefore the intrinsic peak efficiency decreases. When the intrinsic peak efficiency is known, the strength of an unknown source can be estimated.

The analog to digital converter of the spectrometer converts the signal pulse from the photomultiplier into an energy value output. Ideally, the conversion from the signal pulse to the energy value would be linear across all energies. The linearity of 
the spectrometer is used to define this conversion. A linear energy response is advantageous as it allows for a calibration of the output energies using a single parameter called the gain.

The gain parameter affects the spectrometer's output energy value for each energy deposition in the spectrometer. The gain is used to calibrate the spectrometer such that the output energy value matches the deposited energy. The gain can be set using a source of known energy. The mean energy of the measured photopeak is examined to determine if it matches the input gamma ray energy from the source. If the output mean energy does not match the input energy, the gain parameter is changed until the output mean photopeak energy matches the input gamma ray energy. The photopeak from naturally-occurring ${ }^{40} \mathrm{~K}$ or the photopeak from a check source of ${ }^{137} \mathrm{Cs}$ are often used for calibrating the gain. Temperature changes to the electronics within the spectrometer can affect the output energy value of the spectrometer, therefore gain calibrations are periodically required.

As a spectrometer records an event, it is temporarily occupied and unable to record any additional events. The time during which the spectrometer is unable to record events is called the dead time. The dead time is very small for low count rates, therefore it is often negligible for data processing. A dead time correction can be applied for high count rate scenarios.

\subsubsection{Natural Radioactivity}

When conducting a geophysical radiometric survey, a distinctive gamma ray energy from a step in the decay chain of the naturally-occurring isotopes of ${ }^{238} \mathrm{U},{ }^{232} \mathrm{Th}$, and ${ }^{40} \mathrm{~K}$ is measured. The signal from ${ }^{40} \mathrm{~K}$ is directly attributed to its decay into argon-40 $\left({ }^{40} \mathrm{Ar}\right)[7]$. For ${ }^{238} \mathrm{U}$ and ${ }^{232} \mathrm{Th}$, the initial decay step does not result in the emission of a gamma ray. They require the decay of one of their daughter products to produce the distinctive gamma-ray energy. The daughter decay step that is used in geophysical 
surveying has a high gamma branching ratio, therefore producing a larger amount of gamma rays, as well as has a high energy. The measured ${ }^{238} \mathrm{U}$ gamma signal is produced from the decay of its daughter product bismuth-214 $\left({ }^{214} \mathrm{Bi}\right)$. The ${ }^{232} \mathrm{Th}$ signal is produced from the decay of its daughter product thallium-208 $\left({ }^{208} \mathrm{Tl}\right)$.



Figure 1.3: Typical spectrum from a manned aerial radiometric survey. The number of counts, N, per histogram bin per second is shown on the y-axis. The energy, $\mathrm{E}$, is shown on the $\mathrm{x}$-axis. The energy windows typically used in examining the three natural isotopes of ${ }^{238} \mathrm{U},{ }^{232} \mathrm{Th}$, and ${ }^{40} \mathrm{~K}$ are shown. The figure is modified from International Atomic Energy Agency [7].

Figure 1.3 shows a typical spectrum from an manned aerial radiometric survey. Several photopeaks are present within the spectrum and are attributed to the various decay steps of the naturally-occurring radioactive isotopes. The energy window for the photopeak of interest for each of the isotopes is shown. At lower energies, there is a significant background count rate present under the photopeaks. The background is largely caused by scattering of the source gamma rays in the surrounding soil, rock, 
and air. This causes the source gamma rays to loose energy, with the resulting low energy scatter being measured by the spectrometer. At higher energies, the effects of the scattering are significantly reduced, therefore source gamma emissions are more easily distinguished.

In addition to the three previously mentioned naturally-occurring isotopes that are present in the subsurface, cosmic radiation and radioactive atmospheric radon produce a signal in radiometric surveys. The cosmic and radon signals compose part of the background signal of a geophysical exploration survey and methods to remove their effect are explained in Section 3.2.

\subsubsection{Manned Aerial Radiometric Surveying}

Aerial radiometric surveys are traditionally conducted by manned fixed-wing or helicopter aircraft. They are typically flown in a grid pattern over an area of radiological interest.

The survey parameters include the altitude above ground level, the line spacing, and the flight speed. Modifying these parameters affects the spatial resolution of the survey. Flight lines are usually perpendicular to the strike of the main geological feature at altitudes ranging from 40 to $120 \mathrm{~m}$ [7], depending on changes in topography, the height of the trees and the skill of the pilot. Line spacing varies depending on the target and size of the survey area. Reconnaissance survey lines are spaced from 2 to $5 \mathrm{~km}$, regional surveys lines are spaced from $500 \mathrm{~m}$ to $2 \mathrm{~km}$, and detailed survey lines are spaced from 100 to $200 \mathrm{~m}$ [22]. For manned aircraft, flights are typically flown at a speed of $190 \mathrm{~km} / \mathrm{h}[22]$.

Manned aircraft gamma-ray spectrometer systems typically consist of two 16.4 L scintillator spectrometer packages [7]. Each package contains four $10.2 \mathrm{~cm} \times 10.2 \mathrm{~cm} \times 40.6 \mathrm{~cm}$ thallium-doped sodium iodide $(\mathrm{NaI}(\mathrm{Tl}))$ crystals. Often an additional $\mathrm{NaI}(\mathrm{Tl})$ crystal is added on top of the four main crystals to function as 
an upward-looking spectrometer. This crystal is shielded by the four crystals below it, therefore the majority of the counts in this crystal are from atmospheric radon. The data from the upward-looking crystal can then be used to correct data from the four other crystals for background radiation. For quality assurance, the dead time of the spectrometer is required to not exceed $8 \mu$ s per gamma-ray energy deposit, and the FWHM energy resolution at $662 \mathrm{keV}$ should be below $12 \%$. The energy spectrum is recorded every second using 1024 channels from 0 to $3.072 \mathrm{MeV}$ [22].

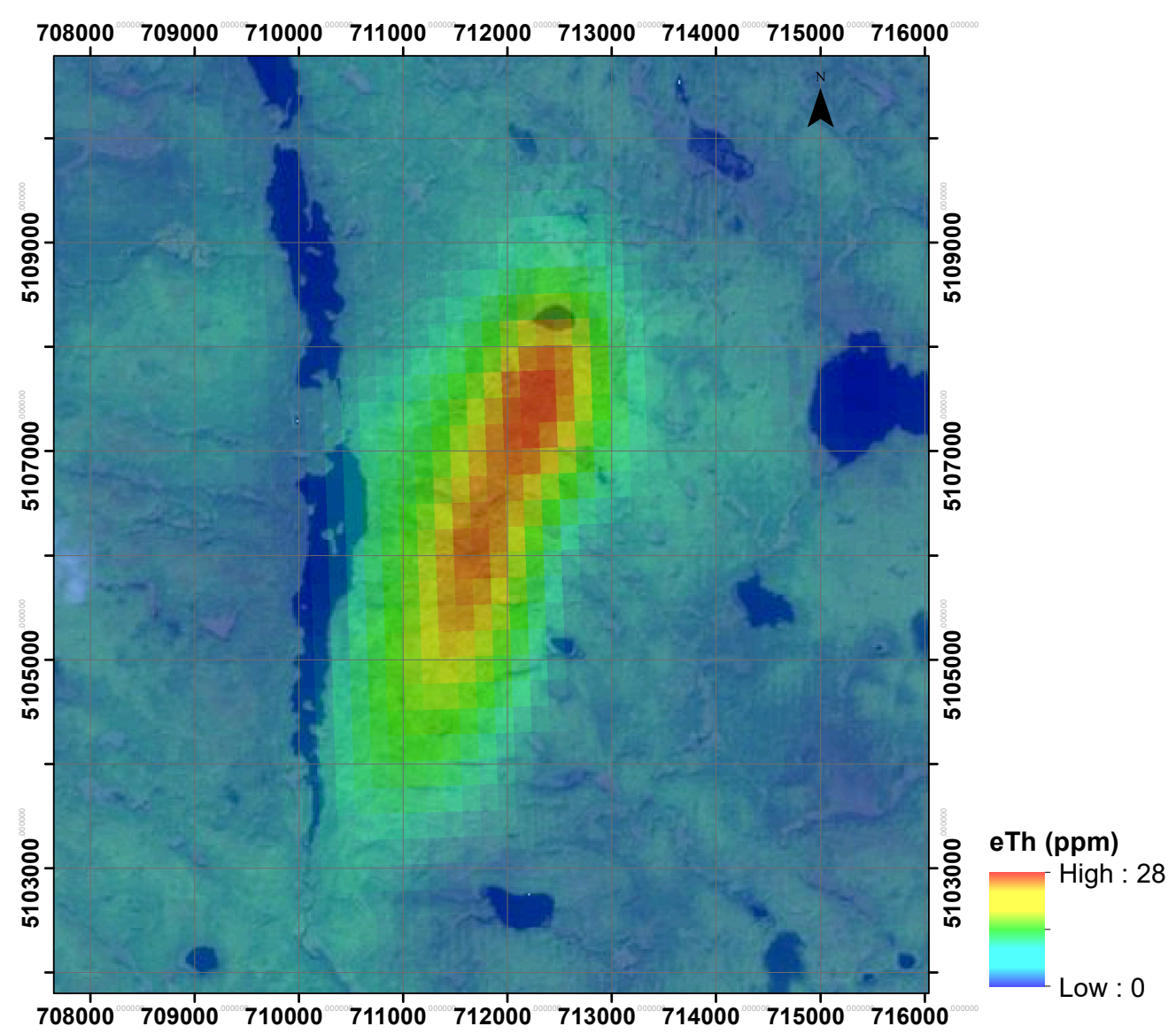

Figure 1.4: Radiometric map of the Allan Lake carbonatite glacial train anomaly in Ontario. The radiometric map is overlaid on a satellite image. The colour scale represent the concentration of equivalent ${ }^{232} \mathrm{Th}$, with red indicating a high anomaly. The grid spacing of the map is $1 \mathrm{~km}$. The data was mapped using open source data available from [26] and [27]. 
Figure 1.4 shows a radiometric map produced from the open source data available from Natural Resources Canada [26] of a survey over the Allan Lake carbonatite glacial train in Ontario. The survey data was collected at an altitude of $120 \mathrm{~m}$ and at a line spacing of $1 \mathrm{~km}[26]$. The map shows a distinct high equivalent thorium anomaly following the glacial train from the Allan Lake carbonatite deposit located on the north end of the anomaly. This data illustrates how radiometric surveying can be used to examine naturally radioactive features that could be used for geophysical exploration purposes.

The flight parameters of this survey are typical for radiometric surveys conducted with a manned aircraft. This results in a map with a resolution of a few hundred meters. While providing large-scale information about the anomaly and its orientation, a more detailed survey has the potential to reduce the area of interest for exploration from kilometres to metres.

\subsubsection{UAS Radiometric Surveying}

Commercial UASs are now being considered for geophysical applications. They are smaller than manned systems, with the advantage of being able to fly lower and slower. This can result in higher resolution maps, improving geological interpretations and providing more detailed information for environmental and security applications. A UAS system can also be easier to deploy than fixed-wing manned aircraft, as they may not need an airport for takeoff, and they can be operated by a person with only a few days of training. A preliminary examination of the contribution of UASs to aerial radiometric surveying of the Allan Lake carbonatite has been performed [28].

UASs are often classified by their takeoff weight. Canadian regulations divide UASs into three categories based on their takeoff weights: $1 \mathrm{~kg}$ or less, above $1 \mathrm{~kg}$ up to $25 \mathrm{~kg}$, and above $25 \mathrm{~kg}$ [29]. Radiation-detecting UASs have been built and tested for each of these takeoff weight classes [14-16, 18-21]. 
The $<1 \mathrm{~kg}$ UASs are limited in their payload capacity. This limits the spectrometer size, and therefore its sensitivity. To effectively determine the location of an anomaly, small spectrometers need to be located closer to the target, and spend more time on each measurement. This can limit the benefits of using a UAS for radiometric surveying.

The $>25 \mathrm{~kg}$ UASs have larger payload capacities. However, they are more complicated to operate and a more complex process is required to obtain regulatory flight permissions.

The 1 to $25 \mathrm{~kg}$ class of UAS provides a balance between the ease-of-use of the smaller UASs, while large enough to carry a spectrometer with an acceptable sensitivity for a reasonable flight time. Traditional aerial gamma spectrometer packages for manned aircraft can weigh $114 \mathrm{~kg}$ [30]. Therefore a smaller gamma spectrometer called the Advanced Radiation Detector for UAS Operations (ARDUO) was designed to make optimal use of the payload capacity for the 1 to $25 \mathrm{~kg}$ class of UAS. 


\section{Chapter 2}

\section{Instrumentation}

This chapter covers the equipment used in this research project. This includes a description of the Responder UAS's flight instruments and capabilities, as well as a description of the ARDUO spectrometer and its laboratory characterization.

\section{$2.1 \quad$ Responder UAS}

The following sections outline the Responder UAS. The Responder UAS is built and commercialized by ING Aviation Robotics Inc. and is composed of a helicopter airframe, a Ground Control Station (GCS), and a radio remote control transmitter [3]. The helicopter airframe is flown using a Pixhawk 1 autopilot. The autopilot is an electronics board containing the software used to fly the UAS. The autopilot is integrated with the flight systems of the UAS, such as the motor and rotor control mechanisms, Global Navigation Satellite System (GNSS) receiver and other sensors. The components of the UAS are described in further detail in their respective subsections.

The UAS is typically flown in a near-autonomous flight mode, where the UAS is capable of completing an defined flight plan from takeoff to landing, following a series of waypoints. The UAS can be flown with a minimum of two operators, and is typically flown with three operators. One operator is responsible for the GCS 
and ARDUO data stream. The GCS operator is also responsible for creating and uploading the flight plan to the UAS autopilot. The second operator is called the Safety Pilot, and is responsible for situations which may require the UAS to be manually flown, aborting the near-autonomous flight plan, using the radio remote control transmitter. The optional third operator is the visual observer. His/Her role is to watching for potential hazards the UAS may encounter and to keep the operations area free from distractions such as public observers. The third operator can also aid the GCS operator with monitoring the ARDUO data stream.

\subsubsection{Airframe}

The Responder airframe is a medium-lift, main- and tail-rotor, battery-powered aircraft. It has a payload capacity of up to $6 \mathrm{~kg}$ and an endurance of up to 40 minutes. This type of airframe was chosen over a fixed-wing airframe as it is capable of vertical takeoffs and landings, and has the ability to hover. In addition, main- and tail-rotor airframes have larger lift capacities and better stability in windy conditions when compared to similar-sized multi-rotor airframes.

The Responder airframe with the ARDUO mounted underneath is shown in Figure 2.1. The electronic components of the Responder are located underneath the back section of the white and blue composite canopy for some protection from the weather. The motor and electronic speed controller are also underneath the canopy, located in the nose of the Responder. The main rotor blades are folded back for transportation.

A Global Navigation Satellite System (GNSS) receiver and compass are mounted near the tail rotor. The two systems are used by the autopilot for positioning and orientation of the UAS. A second GNSS receiver, independent of the UAS autopilot navigation system, is used by the ARDUO and is mounted half-way down the tail boom. 
A sonar altimeter is located halfway down the tail-boom, pointing downwards. The sonar altimeter is used to provide more accurate altitude measurements to the autopilot during takeoff and landing. The sonar altimeter has a limited range of a few meters, and is therefore only used in determining the altitude when near the ground's surface.

A GoPro camera is mounted on the front leg to capture real-time video during flight. The video data stream is sent to the GCS in real time using the telemetry systems (discussed in Section 2.1.3. The video can then be viewed on a screen at the GCS while the UAS is in flight.

A white and blue composite canopy covers the motor, autopilot and electronics boards. This provides some protection from minor precipitation and insects.

A white router box is located underneath the back of the canopy. This is used for the telemetry of the ARDUO data and video link in real time to the GCS.

The batteries, not pictured, would be mounted in the nose of the Responder. The batteries power all the motors and electronics on the Responder airframe. They are also used to power the ARDUO. The Responder uses two 6 cell lithium polymer batteries, with the possibility of flying with four batteries for extended flight times. Each battery weighs $1.9 \mathrm{~kg}$ and has a capacity of $16000 \mathrm{mAh}$ [8]. With two batteries, flight times are approximately $20 \mathrm{~min}$. The batteries are typically changed after each flight.

\subsubsection{Ground Control Station}

The UAS is flown from a ground control station (GCS). The GCS contains the mission planning laptop, a real-time video display, a battery charger and a laptop for control of the ARDUO. 


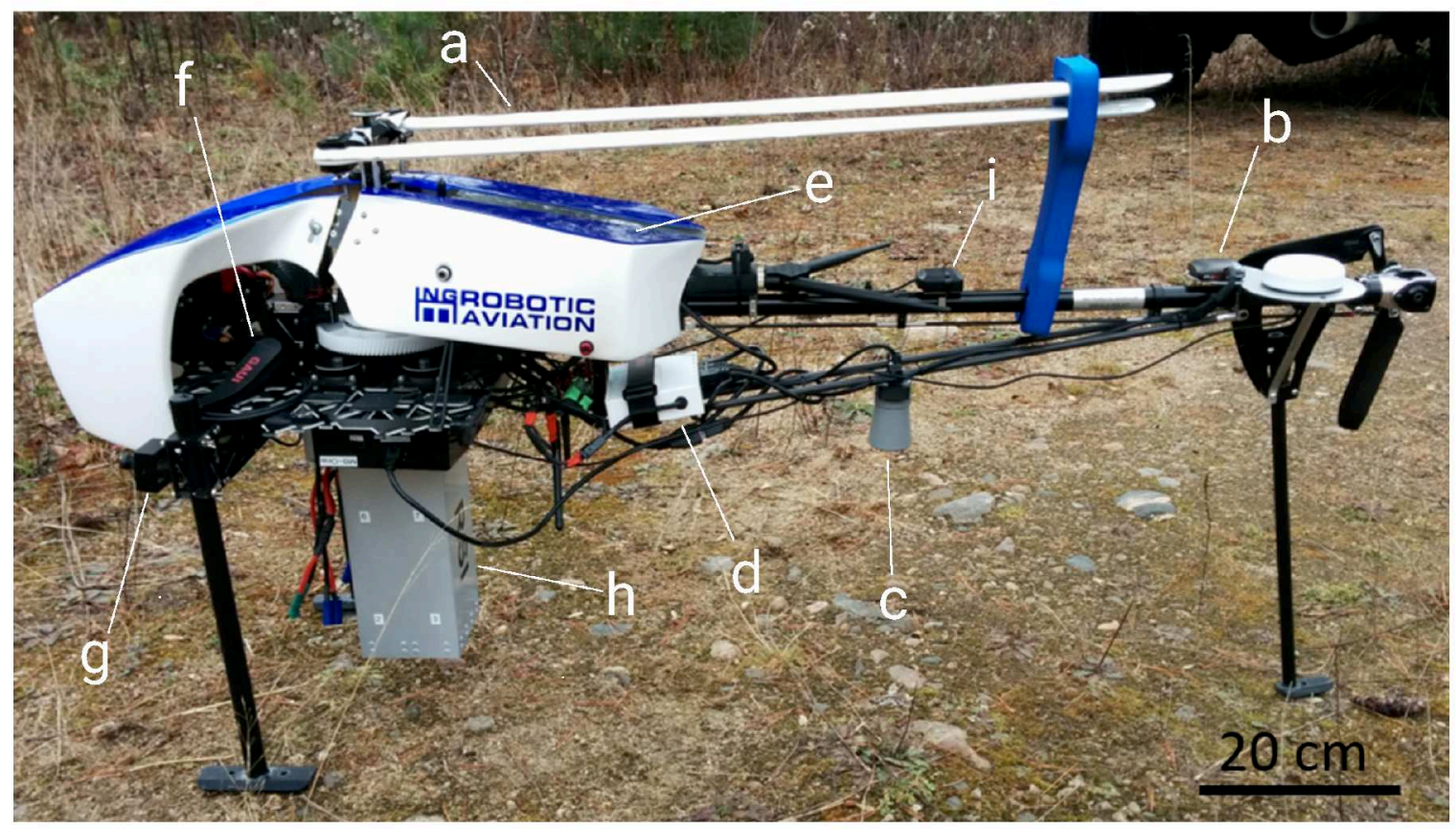

Figure 2.1: The Responder airframe with the ARDUO mounted beneath the main rotor. a) The main rotor blades. They are folded back for transportation. b) The GNSS receiver and compass. c) The sonar altimeter. d) The router for the telemetry of the ARDUO data and video link. e) The autopilot and electronics boards located underneath the white and blue canopy. f) The location where the batteries are attached. The batteries are not pictured. g) The GoPro camera. h) The ARDUO. i) The ARDUO's GNSS receiver that function independently from the Responder's GNSS receiver.

The mission planning laptop uses the ArduPilot Mission Planner software [11]. This software is used to program the flight plans by setting a series of GNSS waypoints and commands that the autopilot follows. Additional commands, such as 'Return to Land', 'Land', 'Altitude Hold', 'Change Speed', are possible from the software. The software is also used to control the autopilot flight parameters (preset by ING Robotic Aviation Inc. [3]), compass and accelerometer calibration settings, and fail safe settings. The fail safes determine the UAS's action if a telemetry connection is lost, the battery charge becomes too low, and if the UAS reaches the virtual geographic GNSS boundary set in the flight plan, also known as a geofence. The laptop is connected through the $0.9 \mathrm{GHz}$ telemetry to the autopilot. Flight plans are uploaded to the autopilot prior to takeoff. During flight, the UAS flight parameters are displayed in 
real time. This includes the GNSS position, orientation in three-dimensions, altitude, battery current and voltage, as well as telemetry signal connectivity.

A screen is set up to display the real time video stream from the GoPro camera mounted on the Responder airframe's front leg. The video uses the $5.8 \mathrm{GHz}$ telemetry frequency. The video can be recorded in real time and replayed after the flight is completed.

A battery charger is included in the GCS to allow for simultaneous flying and battery charging. Charging a set of two batteries takes approximately $20 \mathrm{~min}$. A second set of batteries can therefore be charged while a flight with the UAS is ongoing. Upon completion of the flight, the charged batteries can be swapped with the used batteries on the Responder airframe. The battery changing process takes approximately $10 \mathrm{~min}$. An additional flight can then be conducted with minimal time after the previous flight.

The ARDUO laptop contains the RadAssist software from Radiation Solutions Inc. [5]. The laptop is connected over telemetry to the ARDUO mounted on the Responder airframe. The laptop displays and records the radiation data in real time using the $5.8 \mathrm{GHz}$ frequency. A detailed description of the radiation data real time display can be found in Section 2.2.1. The laptop is also used to conduct the gain calibration of the ARDUO.

The UAS can also be flown by the Safety Pilot in a semi-manual mode using the radio remote control transmitter. This flight mode is typically only required for emergency situations or when the initial flight plan needs to be aborted. The GCS operator is responsible for monitoring the flight and radiation data streams. He/She is also responsible for creating and loading the flight plans. The GCS setup is shown in Figure 2.2. The laptop shows the mission information, and has the $0.9 \mathrm{GHz}$ antenna mounted on the back of the laptop screen. The real-time video screen is mounted on the 'Y'-shaped metal stand. The data stream telemetry router, responsible for 
receiving the real-time video and radiation data, is mounted on a tall branch. The increased height of the router improves the signal transmission from the airframe to the GCS. A generator is used to power the GCS in remote locations. Three operators are present for the pre-flight preparations.

\section{Radio Remote Control Transmitter}

The remote control transmitter is the Futaba T8J Radio Control System, shown in Figure 2.3. The transmitter is used by the Safety Pilot to manually control the UAS in emergency situations. The controller has a switch that sends a signal to power on and shut off the motor. Another switch is used to change the flight mode from auto (semi-autonomous flight following the mission plan), loiter (semi-manual flight using the remote control transmitter), or stabilized (fully manual flight using the remote control transmitter). These flight modes can also be changed using the GCS laptop. The two joysticks are used for controlling the movement of the UAS in loiter or stabilized flight modes.

The Safety Pilot is in control of the remote control transmitter. The safety pilot is responsible for the takeoff and landing procedures, in addition to emergency flight procedures. There is constant communication between the safety pilot and GCS operator to relay flight information about the UAS. Additional operators can be used as visual observers, with the role of visually assessing the survey area for hazards before and during the flight.

\subsubsection{Telemetry Systems}

The three telemetry systems of the Responder each use a different frequency for communications between the airframe systems and the ground systems. 


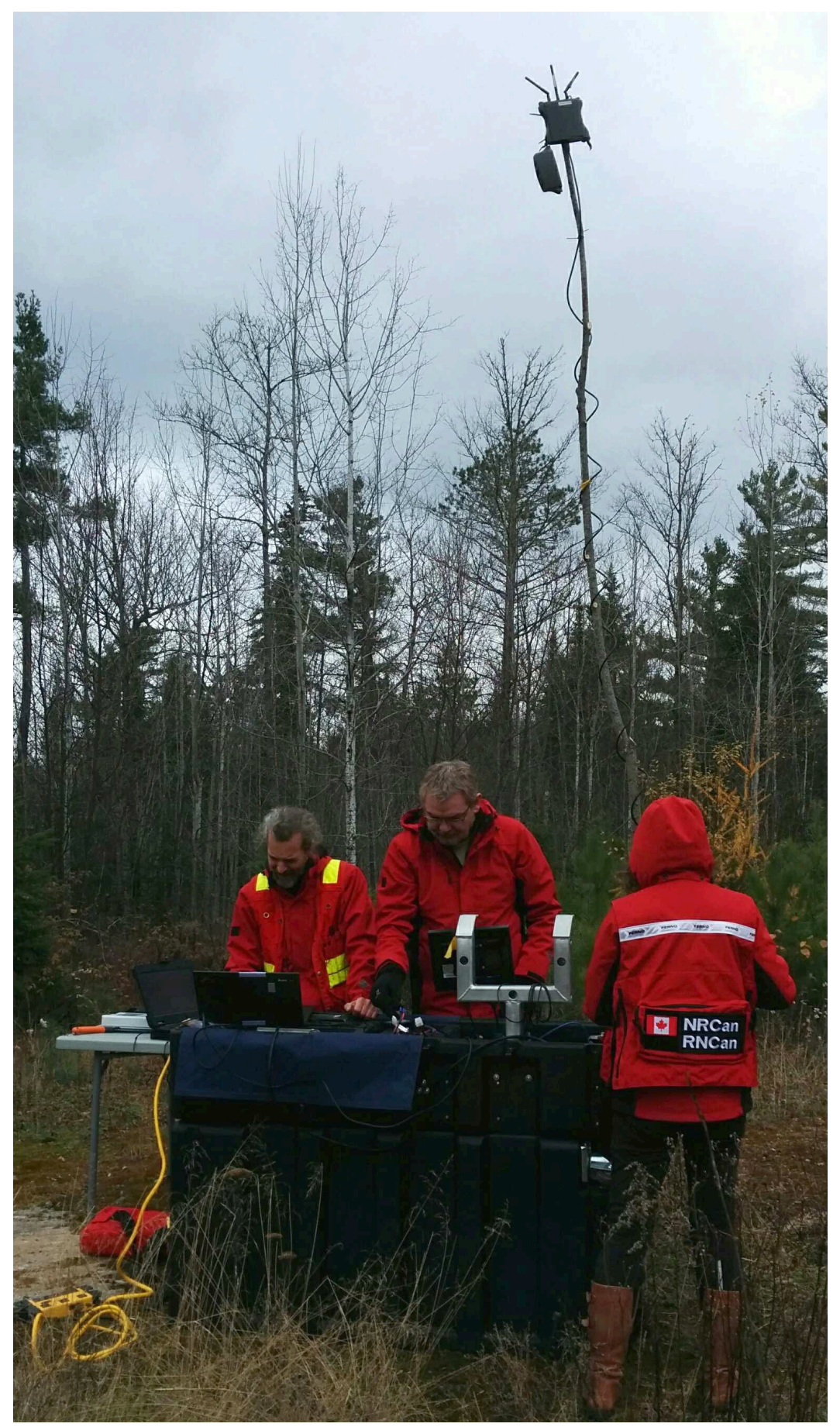

Figure 2.2: The GCS. The mission information is displayed on the GCS laptop. A video screen mounted on the 'Y'-shaped metal stand shows the real-time video from the GoPro camera mounted on the Responder's front leg. A branch was used to elevate the data stream transmission receiver.

The GCS mission planning laptop information is transferred at a frequency of $0.9 \mathrm{GHz}$. This is the lowest frequency used and can therefore transmit information 


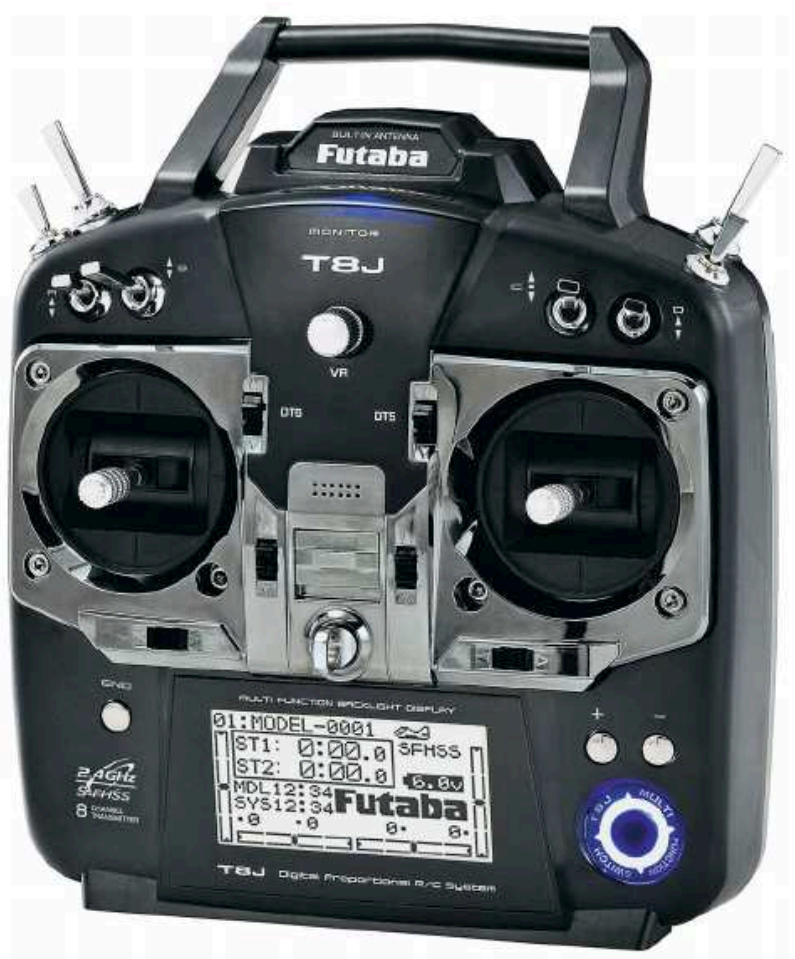

Figure 2.3: The Futaba T8J radio control transmitter [8]. The switches are programmed to change the flight modes, as well as turn the motor on and off. The sticks control the movement of the Responder when set to the semi-manual flight mode. Dimensions of the controller are $43 \mathrm{~cm} \times 25 \mathrm{~cm} \times 13 \mathrm{~cm}$.

over the largest distance. Low frequencies are also more limited in the amount of information they can transfer. The data from the mission planning laptop is relatively small, therefore there are no issues with the quantity of data being transferred.

The data stream, including the video link and radiation data, is transferred at a frequency of $5.8 \mathrm{GHz}$. This is the highest frequency used. This frequency was used to be able to transmit the larger amount of data from the video stream and radiation data. It has the lowest range out of the frequencies used.

The remote control transmitter uses a frequency of $2.4 \mathrm{GHz}$. This frequency is capable of transferring the commands from the remote control transmitter to the UAS. The range is less than the GCS $0.9 \mathrm{GHz}$ range, but greater than the data stream $5.8 \mathrm{GHz}$ range. 
The use of different frequencies for each component, the GCS, data stream, and remote control transmitter, allows for the potential loss of one of the signals while still maintaining control over the UAS.

\subsubsection{Autopilot}

The UAS's PixHawk 1 flight controller, also known as the autopilot, controls the flight of the UAS. The autopilot uses a custom version of the ArduCopter software by ING Robotic Aviation Inc. [3]. The autopilot is shown in Figure 2.4. The autopilot contains a gyroscope, an accelerometer/magnetometer, a 3-axis accelerometer/gyroscope, and a barometer within its housing; and is connected to the Responder airframe's compass, sonar altimeter, GNSS receiver and the motors controlling the rotor blades. The sensors are used to determine the position, velocity and attitude of the UAS. The information is then used to control the movement of the UAS to follow the waypoints of the flight plan.

The autopilot also logs the position and orientation of the system during flight [31]. The UAS's position and orientation can be defined by the UAS's state parameters. The parameters often include the geographic position in latitude and longitude, the position relative to fixed location on the Earth's surface, the three-dimensional airspeed, the aerodynamics angles, the attitude angles (heading, pitch, roll), the threedimensional acceleration, the three-dimensional angular rotation rates.

\section{Extended Kalman Filter}

The autopilot uses each of its sensors to determine the UAS's state. Each sensor can effect one or several of the UAS state parameters. As well, different sensors can

provide information on the same UAS state. For example, the accelerometer reading provides information about changes in the velocity of the UAS, and therefore can 




(a)

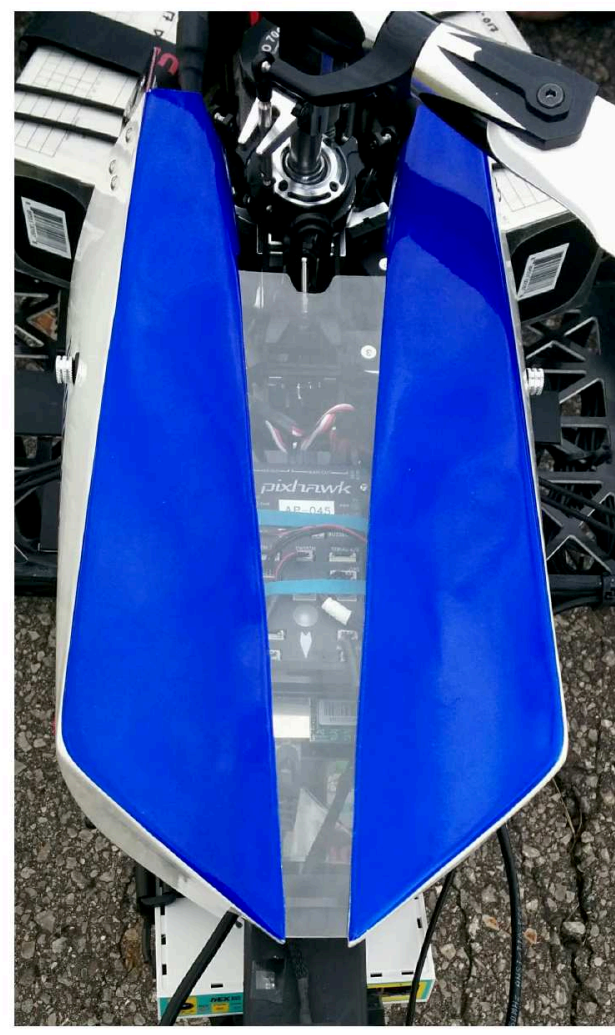

(b)

Figure 2.4: a) The Pixhawk 1 autopilot [8]. Dimensions of the Pixhawk 1 are $5 \mathrm{~cm} \times 8.15 \mathrm{~cm} \times 1.55 \mathrm{~cm}$. b) Top-down view of the Responder. The main rotor is located at the top of the picture. The Pixhawk autopilot is mounted beneath the canopy and can be seen through the clear plastic cover.

also provide information about the position of the UAS. The GNSS receiver also provides information on the position of the UAS. To integrate each sensor's information, with their various measurement rates, drifts, resolutions and accuracies, an extended Kalman filter (EKF) is used [32]. This allows for a more accurate determination of the state of the UAS, as the data from all the sensors are used as an input. The ArduCopter EKF uses a 22 state system, with additional state parameters for the gyroscope biases, accelerometer bias, compass biases and for the three components of the Earth's magnetic field vector [33].

The EKF is uses a recursive non-linear method to predict the state of a system. It involves two major steps; the first step to predict the future state of the system, 
and the second step to update the predicted state using sensor measurements. The results from the update step are then fed back into the prediction algorithm and the process is reiterated. The EKF uses both the sensor measurements as well as errors, calculating an error covariance matrix for each step of the process. The EKF assumes a Gaussian distribution for the sensor measurements and errors. The EKF results for the positioning and orientation of the UAS are logged every $0.02 \mathrm{~s}$.

\subsection{Advanced Radiation Detector For Unmanned Aircraft System Operations}

Using advanced electron and gamma radiation transport simulation software [34], NRCan designed the Advanced Radiation Detector for Unmanned Aircraft System Operations (ARDUO). NRCan's design included determining the scintillating crystal material, light collection technology and the spectrometer's size. NRCan's design also included optimization of the self-shielding arrangement of the crystals which creates directionality in the spectrometer [35]. The individual $\mathrm{CsI}(\mathrm{Tl})$ crystals coupled with silicon photomultipliers were developed and built by Scionix [4]. Radiation Solutions Inc.(RSI) [5] designed the miniaturized pulse-shaping and digitization electronics for the ARDUO. In addition, they designed the external housing that contains the crystals, electronics and associated components. RSI has provided their RadAssist software to view the ARDUO's data in real time. As well, RSI has integrated NRCan's real-time direction reconstruction algorithms into their display.

The ARDUO consists of eight $2.8 \mathrm{~cm} \times 2.8 \mathrm{~cm} \times 5.6 \mathrm{~cm} \mathrm{CsI}(\mathrm{Tl})$ crystals in a selfshielding arrangement, resulting in a sensitive volume of $0.35 \mathrm{~L}$. The ARDUO features GNSS receiver and antenna that are independent from those of the Responder. The GNSS system is used to collect independent positioning information, as well as to trigger the radiation data accumulation process using the GNSS time. The position 
and radiation data is collected every second. The radiation data consists of eight 1024channel energy spectra (one spectrum from each crystal), and each data point is saved and tagged with a position and time-stamp from the GNSS receiver at a sampling rate of once per second. The 1024 channels cover an energy range of 0 to $3.072 \mathrm{MeV}$, with the last channel dedicated to counting energies above $3.072 \mathrm{MeV}$ that are due to cosmic radiation. Each channel, with the exception of the cosmic channel, is $3 \mathrm{keV}$ in width.

The ARDUO is integrated into the Responder power and telemetry systems. The ARDUO is connected and powered using the batteries from the Responder. The radiation data stream is sent to a laptop connected to the GCS using the $5.8 \mathrm{GHz}$ link for real-time viewing. The ARDUO is not currently connected to the Responder's positioning system for real-time integration. In post-acquisition processing, the ARDUO radiation data stream and the Responder positioning data stream can be integrated. This process is further explained in Section 3.2.

Future work could be conducted to integrate the positioning and orientation data from the UAS autopilot with the radiation data from the ARDUO in real time. This would decrease data post-acquisition processing time as the data stream integration step would already be completed. Additionally, real-time integration would allow for the use of the director vectors calculated from the ARDUO to potentially be used to guide the UAS during flight.

The Responder airframe with the ARDUO is shown in Figure 2.1. The ARDUO is mounted underneath the main rotor of the Responder where the center of gravity is located. The ARDUO is connected to the batteries as well as to the white router for real-time telemetry. The ARDUO's independent GNSS receiver is located halfway down the tail-boom. 


\subsubsection{Self-Shielding Crystal Configuration}

Figure 2.5a shows the ARDUO in a laboratory setting. The ARDUO has two sections within its housing. The top section contains the electronics for pulse-shaping and digitization of the signal from the eight crystals. The bottom section contains the scintillation crystals.

Figure 2.5b shows the crystal layout. The crystals are arranged in a rectangular array with two layers of four crystals. This arrangement provides three dimensional self-shielding, where two crystal layers are present at any angle in space. The first layer of crystals will absorb and detect a large amount of the incident gamma radiation producing a relatively high count rate, while shielding the second layer of crystals which will have a relatively lower count rate. The algorithms used to reconstruct the directions from the count rate ratios are explained in Section 3.2. A representative direction vector is also shown in Figure 2.5b. The direction vector is defined by an azimuth angle from the $0^{\circ}$ axis, and elevation angle from the horizon. The ARDUO is oriented such that the $0^{\circ}$ azimuth axis is aligned with the nose of the Responder airframe.

\section{Real Time Radiation Data Display}

Incorporating the telemetry of the Responder, the ARDUO control software displays the self-shielding direction vector to the operator in real time. A screenshot of the real-time display is shown in Figure 2.6. The display is updated every second when a new radiation data point is collected. The top left display shows the number of counts per second in the energy spectrum versus time. Directly below this display is a one-second energy spectrum. The bottom display shows a map of each data point location coloured according to the total count rate. The top right display shows the direction information, with the white ellipse representing the calculated direction 


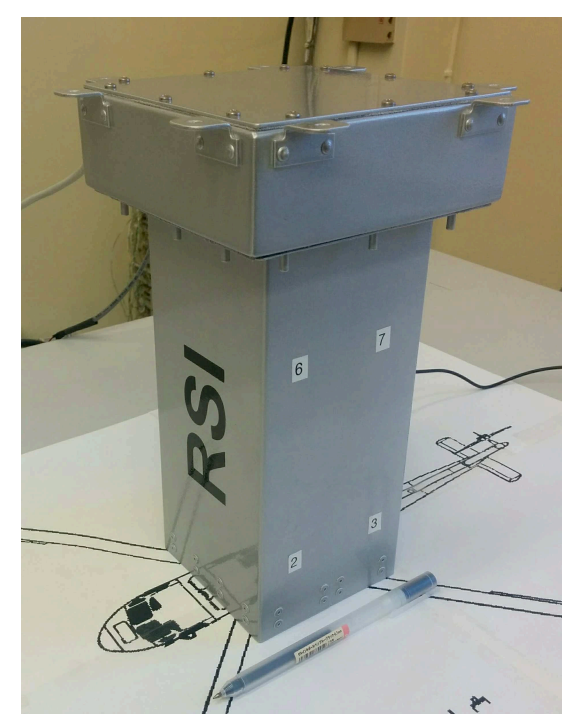

(a)

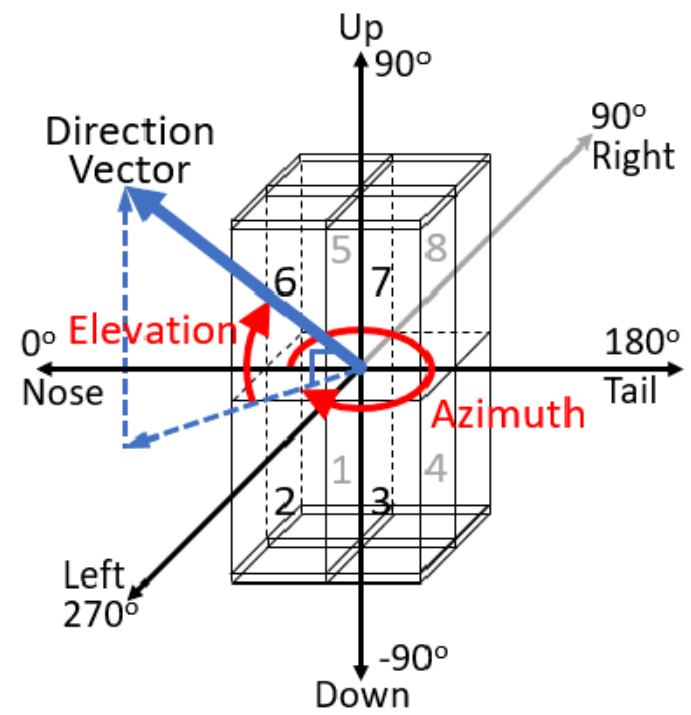

(b)

Figure 2.5: a) The ARDUO in a laboratory setting. The bottom section of the ARDUO housing has dimensions of $10 \mathrm{~cm} \times 10 \mathrm{~cm} \times 20 \mathrm{~cm}$. b) The ARDUO crystal arrangement and direction vector orientation. The individual crystals are numbered from 1 to 8 . The nose and tail directions are indicated. The elevation and azimuth angles for a representative deconstructed direction vector are shown.

vector relative to the UAS. In the screenshot provided, it can be seen in the direction display that the source is located towards the right with respect to the UAS heading, at an approximate $-45^{\circ}$ angle downwards. The UAS flight direction is indicated by the overlaid black arrow in the map display. This arrow is not displayed in real time as the UAS heading can be seen as it is flying. The reconstructed direction shown in the upper right display corresponds well to the true source location indicated by the black star in the map display that is located to the right of the UAS heading arrow. The source location is not typically shown in the map display as it is not usually known. The operator can also use the energy spectrum information in real time for isotope identification. 

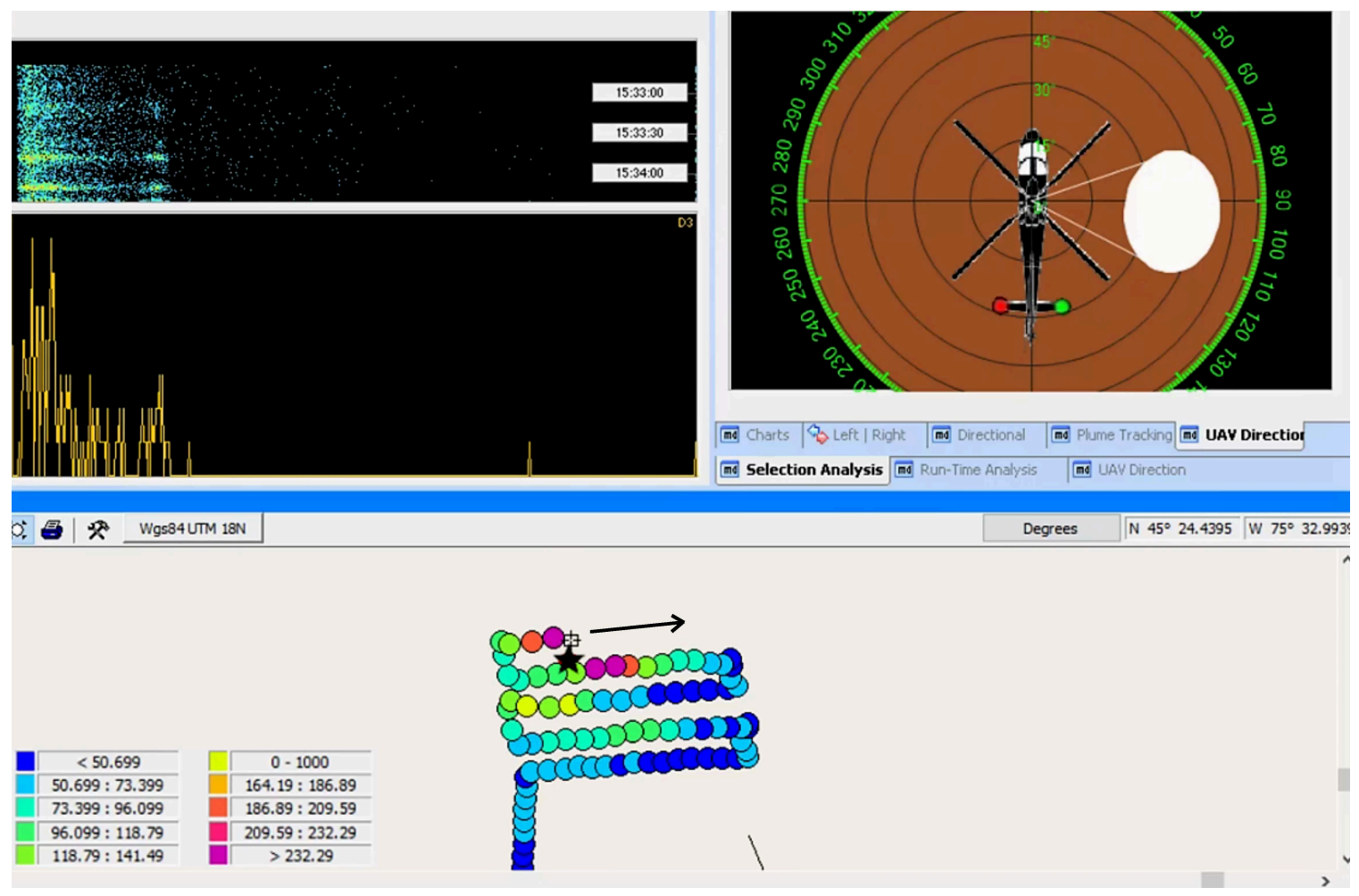

Figure 2.6: Real-time data view during an aerial survey. The top left screen shows time on the vertical axis, energy on the horizontal axis, and counts using the colour scale. The horizontal axis energy range is from 0 to $3.072 \mathrm{MeV}$. The middle left screen shows an energy spectrum, and the top right screen shows the direction reconstruction; both displays are refreshed every second. The bottom screen shows the GNSS location of each data point on a colour scale from blue (low count rate) to purple (high count rate). Here a black star has been overlaid to show the true source location. Unless the source location is known, it is not usually available in real time.

\subsubsection{Linearity}

Linearity is the property of a spectrometer to give an output energy value which is linear in response to the input quantity. A linear energy response is desirable because it allows for calibration of the spectrometer's energy scale using a single slope parameter called the gain. The linearity of the ARDUO response was examined using a variety of low-activity check sources of different isotopes to cover a range of photopeak energies. The list of isotopes and the photopeak energies used for the analysis can be found in Table 2.1. Before each measurement, the ARDUO gain was 
calibrated at an energy of $0.662 \mathrm{MeV}$. The gain calibration parameter is explained in Section 2.2.4. Each check source was then placed against each crystal of the ARDUO for a period of 5 min to accumulate a statistically significant amount of counts within the photopeak of interest. The eight crystal spectra were plotted for each check source.

Figure 2.7 shows an example spectrum from crystal 1 of the ARDUO from the ${ }^{152} \mathrm{Eu}$ check source. The photopeaks in the spectra were fitted using a Gaussian distribution plus a linear function. The location, width, and magnitude of the Gaussian peak, as well as the slope and intercept of the linear function were allowed to vary. The Gaussian distribution defines the photopeak, while the linear function defines the background scattering from natural sources and Compton scattering from higher energy sources. The total fit function is overlaid on the filled green spectrum of the signal from the check source. The fit function has also been broken down into its separate components and both the Gaussian distribution component and the linear component are overlaid on the signal spectrum.

Table 2.1: List of isotopes and their associated photopeak energies that were used for the linearity and energy resolution tests.

\begin{tabular}{cc}
\hline Isotope & Photopeak Energy $(\mathrm{MeV})$ \\
\hline${ }^{133} \mathrm{Ba}$ & 0.081 \\
${ }^{152} \mathrm{Eu}$ & $0.121,0.244,0.344$ \\
${ }^{137} \mathrm{Cs}$ & 0.662 \\
${ }^{54} \mathrm{Mn}$ & 0.835 \\
${ }^{60} \mathrm{Co}$ & $1.173,1.332$ \\
\hline
\end{tabular}

The mean of the Gaussian fit, with the linear background removed, was then determined and used for the linearity analysis. A plot of the mean photopeak energies for each crystal versus the true photopeak energy is shown in Figure 2.8. The blue line is a 1:1 line. The mean energies should plot along this line to indicate that the energy 


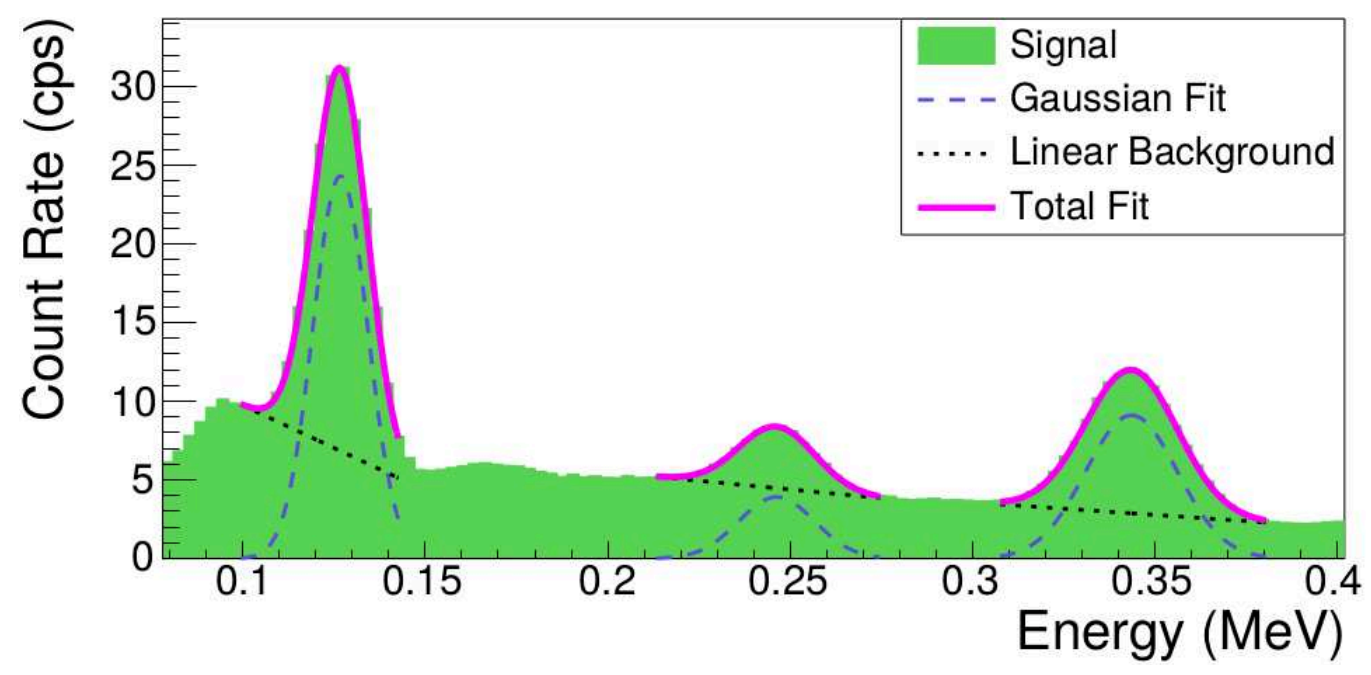

Figure 2.7: Spectrum collected from crystal 1 of the ARDUO using a ${ }^{152} \mathrm{Eu}$ check source. The number of counts, $\mathrm{N}$, per second is plotted on the y-axis. The energy, $\mathrm{E}$, is plotted on the $\mathrm{x}$-axis. The signal from the ${ }^{152} \mathrm{Eu}$ check source is the filled area. The linear background, Gaussian and total fits are shown by the dotted, dashed and solid lines. The fits are overlaid on the photopeaks that were used for the linearity analysis.

measured by the ARDUO corresponds to the true energy. There is generally a good correspondence between the mean energies and the 1:1 line, with small deviations at higher energy values.

\subsubsection{Full Width Half Maximum Energy Resolution}

The FWHM energy resolution determines the quality of the instrument's performance. This resolution is dependent on the energy deposited in the spectrometer and should follow a characteristic curve that defines the statistical broadening of the photopeaks.

The ARDUO's FWHM energy resolution was examined over a range of energies using the check sources from the linearity tests. The list of isotopes and photopeak energies can be found in Table 2.1. Using the spectra fit results from the linearity tests, the FWHM energy resolution for a given energy, $R_{\mathrm{FWHM}}(E)(\%)$, is calculated 


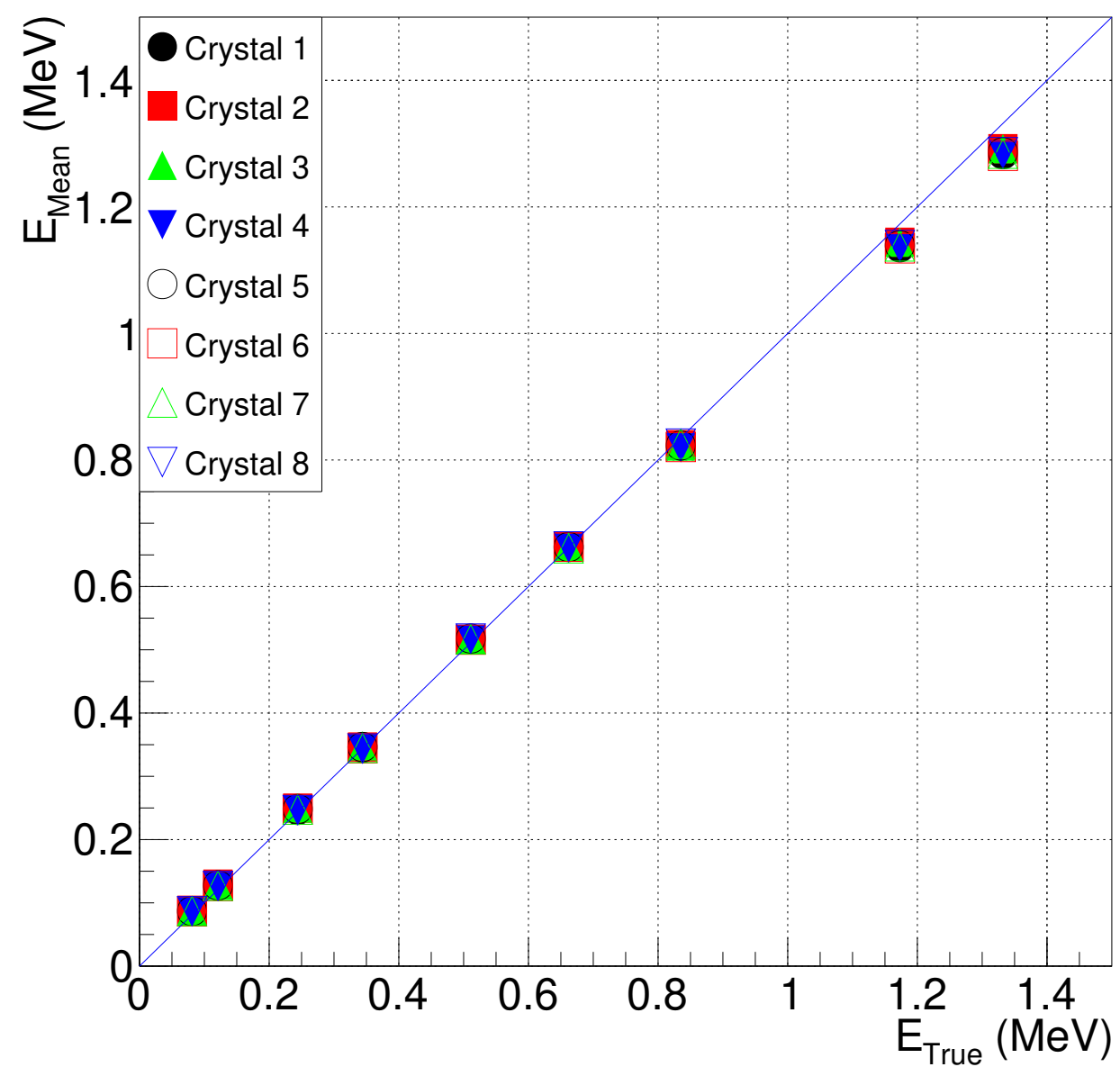

Figure 2.8: The linearity of the ARDUO when calibrated at $0.662 \mathrm{MeV}$. The measured energy, $\mathrm{E}_{\mathrm{Mean}}$, is the mean energy from the photopeak fit using a Gaussian function and subtracting a linear background. $\mathrm{E}_{\text {True }}$ is the true energy of the photopeak. The measured energy for each crystal of the ARDUO is plotted following the symbols in the legend. The blue line is a 1:1 line where the true energy is equal to the mean energy.

using the mean energy, $E_{\text {Mean }}(\mathrm{MeV})$, and the standard deviation, $\sigma(\mathrm{MeV})$, of the Gaussian fit [24],

$$
R_{\mathrm{FWhM}}(E)=\frac{2.35 \sigma}{E_{\text {Mean }}} .
$$

The results for each crystal from the FWHM energy resolution tests are shown in Figure 2.9. The FWHM energy resolution was plotted versus the true energy for each 
crystal. This curve is characteristic of the statistical broadening of the photopeaks, which is predicted by,

$$
R_{\mathrm{FWhM}}(E)=\frac{K}{\sqrt{E}},
$$

where $R_{\mathrm{FWHM}}(E)$ is the FWHM resolution (\%), $E$ is the photopeak energy $(\mathrm{MeV})$, $K$ is a proportionality constant $\left(\mathrm{MeV}^{1 / 2}\right)$ [24]. At an energy of $0.662 \mathrm{MeV}$, the FWHM energy resolution for the eight ARDUO crystals is approximately 7\%. This is within acceptable operational values as described by the International Atomic Energy Agency [7], and is better than the typical value for a scintillation spectrometer of $8 \%$. The proportionality constant for each crystal is determined from a fit of the FWHM resolution versus true energy plot for each crystal. This constant can then be used to calculate the energy resolution for the ARDUO at any energy. The proportionality constants for the ARDUO are listed in Table 2.2.

Table 2.2: Proportionality constants for each crystal of the ARDUO calculated from a fit of the FWHM energy resolution versus true energy plot.

\begin{tabular}{cc}
\hline Crystal Number & $K\left(\mathrm{MeV}^{1 / 2}\right)$ \\
\hline 1 & 4.944 \\
2 & 4.897 \\
3 & 4.963 \\
4 & 4.947 \\
5 & 4.946 \\
6 & 4.876 \\
7 & 4.820 \\
8 & 4.935 \\
\hline
\end{tabular}




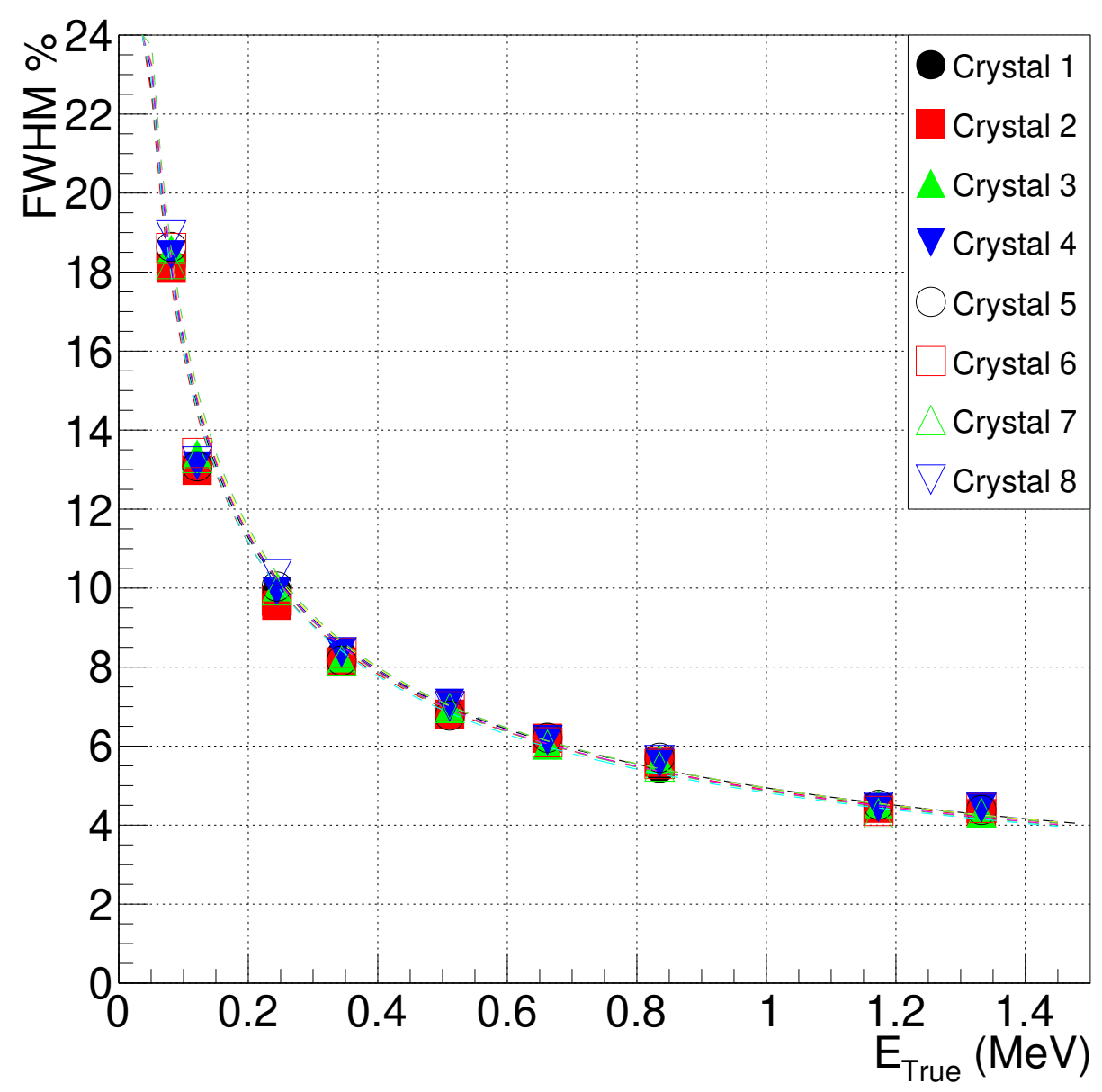

Figure 2.9: The measured FWHM energy resolution for each crystal of the ARDUO when calibrated at $0.662 \mathrm{MeV}$. $\mathrm{E}_{\text {True }}$ is the true energy of the photopeak. The dashed lines are the fitted functions for each crystal using Equation 2.2. Isotopes used in the analysis are listed in Table 2.1.

\subsubsection{Gain}

Over time, the energy response can shift such that the measured energy does not correspond to the true energy. This is primarily due to changes in a spectrometer's internal temperature. The gain parameter is used to calibrate the spectrometer's energy response. This is a parameter that shifts the output energy value to correspond to the true energy deposited in the spectrometer. 
Some gamma spectrometers contain self-stabilizing features that adjust the gain calibration using the photopeaks from natural sources as a reference. This is useful for geophysical surveys where natural sources are dominant. For surveys over higher activity man-made radiation sources, the natural signal from natural sources can be masked, causing the self-stabilizing gain calibration to fail. The self-stabilization feature is not included in the ARDUO, therefore tests were conducted to determine how the gain parameter drifts over time. These tests could then be used to determine operational requirements for calibrating the spectrometer.

To determine the dependence of the gain over time, the ARDUO was left running in the laboratory for a four hour period with two ${ }^{137} \mathrm{Cs}$ check sources placed in the center of the ARDUO on opposite faces. The gain parameter was calibrated at an energy of $0.662 \mathrm{MeV}$ immediately after startup. Measurements were collected following the gain calibration. A thermocouple was placed in the top housing of the spectrometer by the electronics boards to determine the internal temperature. The photopeak mean energy was calculated every 10 min using a 5 min accumulation time. The mean photopeak energy for each crystal and internal temperature within the top housing of the ARDUO, are shown in Figure 2.10 versus time since the ARDUO was powered on. The process of connecting the laptop to the ARDUO and setting the gain calibration took approximately $10 \mathrm{~min}$. A 5 min period was given after the initial gain setting and before the first data point accumulation. Each point is plotted at the end of the 5 min accumulation period. The total data acquisition period was 3 hours.

As shown in Figure 2.10, the photopeak energy decreases for all eight crystals as time progresses, indicating that the gain is drifting over time. This drift varies for each crystal, with the bottom four crystals, number 1 to 4, drifting less than the top crystals, number 5 to 8 . This is expected as the top crystals are located closer to the electronics and are therefore more exposed to temperature variations from the electronic components. 
After the ARDUO is turned on, it takes approximately 100 minutes for the internal temperature of the top of the housing to stabilize. The largest drift rates occur within this 100 minute period, indicating that the change in internal temperature is affecting the gain. After the internal temperature in the top of the housing near the electronics stabilizes, the photopeak continues to drift, but at a slower rate. This is likely due to continued temperature changes in the bottom housing near the crystals. The thermocouple was only located in the top section of the housing, therefore temperature changes in the bottom section of the housing where the crystals are located is not known. The continual drift of the photopeak after 100 min may indicate that the internal temperature in the bottom housing has not reach equilibrium and that the temperature is still continuing to change.

To deal with the gain drift during flight operations, a ${ }^{137} \mathrm{Cs}$ check source is used to set the gain before each flight. The ARDUO is also powered for a period of approximately 40 min before calibrating the gain and takeoff. This allows the ARDUO electronics to warmup, and prevents some of the higher rate drift from affecting the data acquisition. As UAS flight times are approximately $20 \mathrm{~min}$, this results in a drift of approximately $1 \%$ at an energy of $0.662 \mathrm{MeV}$ over the duration of the flight. Radiometric standards state that the ${ }^{40} \mathrm{~K}$ and ${ }^{232} \mathrm{Th}$ peaks should not drift more than 2 channels over the course of a survey [7]. As traditional instruments have channel widths of approximately $17 \mathrm{keV}$, this corresponds to an approximate peak drift of $2 \%$. The ARDUO's drift during flight is within this range. To minimize the effect of the gain drift, the gain is calibrated at the beginning of each flight.

\subsubsection{Point Source Intrinsic Photopeak Efficiency}

The intrinsic photopeak efficiency of the ARDUO was estimated for a point source directly below the ARDUO from a series of laboratory measurements using a calibrated 


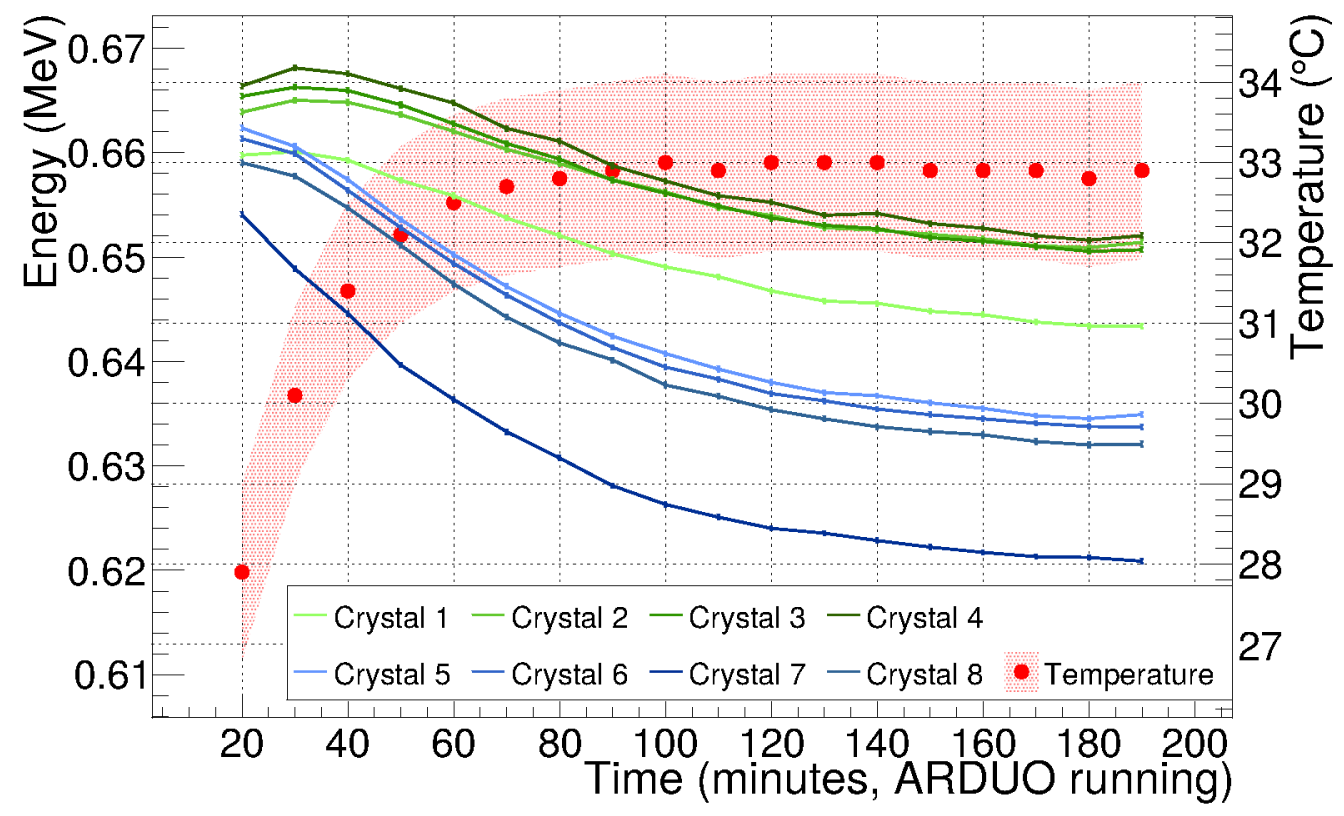

Figure 2.10: The mean photopeak energy of each crystal of the ARDUO from two ${ }^{137} \mathrm{Cs}$ check sources placed in the center of opposing faces of the ARDUO versus time. The internal temperature recorded with a thermocouple which had been placed inside the ARDUO housing near the electronics boards is also plotted versus time. The mean photopeak energy of each crystal calculated from an accumulation of $5 \mathrm{~min}$ of data is shown by the blue and green lines. The red circles and red shaded area show the recorded internal temperature and its error.

${ }^{137} \mathrm{Cs}$ source. This provides a preliminary investigation into the intrinsic photopeak efficiency of the ARDUO. A three dimensional characterization of the intrinsic photopeak efficiency could be completed in future work. This three dimensional characterization would require a series of measurements at various azimuth and elevation angles and an integration of the effective area of the ARDUO for each measurement location. The effects of the UAS airframe would also require characterization. This analysis is outside the scope of this thesis.

The measurements were taken at the NRCan Geomagnetic Laboratory in the east end of Ottawa. The experimental setup is shown in Figure 2.11. The ARDUO was placed on its side with its vertical axis rotated to a horizontal orientation. The UAS airframe was not present. A calibrated ${ }^{137} \mathrm{Cs}$ source (not shown in Figure 2.11) with 
an activity at the time of the experiment of $3.2 \pm 0.1 \mathrm{MBq}$ was placed along the ARDUO's vertical axis at a $-90^{\circ}$ elevation angle. A laser level was used to align the source to the ARDUO's vertical axis, and a laser range-finder was used to determine the distance from the ARDUO's center to the source. The ARDUO was powered through shore power and connected directly via Ethernet cable to the data analysis laptop containing the RadAssist software.

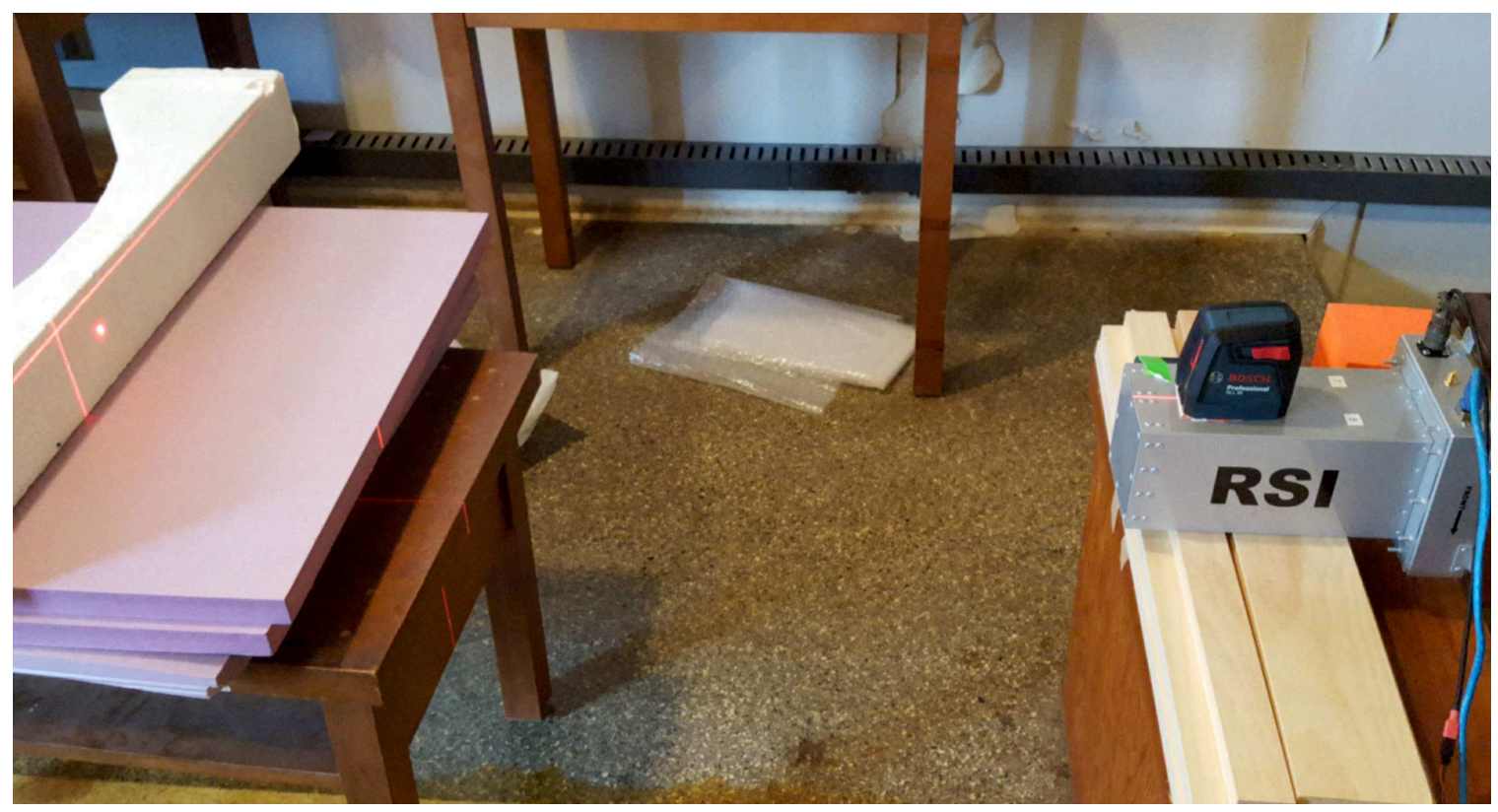

Figure 2.11: Experimental setup for the intrinsic photopeak efficiency measurements. The source (not pictured) would be placed on the foam structure to the left of the figure. A laser range-finder was used to determine the distance from the source to the center of the ARDUO. A laser level was used to align the source to the center axis of the ARDUO.

Measurements were taken at source-ARDUO distances from $1 \mathrm{~m}$ to $4 \mathrm{~m}$, at $1 \mathrm{~m}$ intervals. Two measurements were taken at each location. For the $1 \mathrm{~m}$ and $2 \mathrm{~m}$ locations, 5 min measurements were taken. At the farther distances of $3 \mathrm{~m}$ and $4 \mathrm{~m}$ a longer measurement time of 7 min was used to obtain a sufficient number of counts for good statistics at these farther distances. The measured count rate at each location was normalized to the measurement duration. 
Using this experimental setup, the point source intrinsic photopeak efficiency, $\epsilon_{\text {int }}(E)$, was calculated using

$$
\epsilon_{\text {int }}(E)=\frac{n_{\mathrm{p}}}{\Phi},
$$

where $n_{\mathrm{p}}$ is the measured photopeak count rate with the background count rate subtracted (cps), and $\Phi$ is the calculated theoretical count rate (cps) at the center of the ARDUO. $\Phi$ was estimated using the equation,

$$
\Phi=A \cdot \mathrm{BR} \cdot \frac{A_{\mathrm{ARDUO}}}{4 \pi R^{2}} \cdot e^{-\mu_{\mathrm{air}}(E) R},
$$

where $A$ is the strength of the point source in $\mathrm{Bq}, \mathrm{BR}$ is the branching ratio for the isotope photopeak energy, $A_{\text {ARDUO }}$ is the cross-sectional area of the ARDUO along a plane that is perpendicular to the source location $\left(\mathrm{m}^{2}\right), R$ is the distance from the center of the ARDUO to the point source $(\mathrm{m})$, and $\mu_{\text {air }}(E)$ is the attenuation coefficient of air $\left(\mathrm{m}^{-1}\right)$ at energy $E(\mathrm{MeV})$. The attenuation coefficient of dry air was determined from the National Institute of Standards and Technology data, and a value of $0.00925 \mathrm{~m}^{-1}$ was used for the efficiency calculations [36].

The measured photopeak count rate, $n_{p}$, was determined by summing the count rates within a range of three standard deviations as defined by the FWHM of the mean photopeak energy for each crystal. The background count rate in the photopeak energy window was subtracted from each measured count rate. The results from each measurement are shown in Table 2.3. The average intrinsic photopeak efficiency at an energy of $0.662 \mathrm{MeV}$ is $32.0 \% \pm 0.9 \%$, using the root mean squared deviation as the error. The point source intrinsic photopeak efficiency will be used in the process to be described in Section 3.2.10 for the source strength estimation of a single ${ }^{137} \mathrm{Cs}$ point source (Section 4.2.1). 
Table 2.3: Intrinsic photopeak efficiency measurements.

\begin{tabular}{ccccc}
\hline Run ID & $R(\mathrm{~m})$ & $n(\mathrm{cps})$ & $\Phi(\mathrm{cps})$ & $\epsilon_{\text {int }}$ at $0.662 \mathrm{MeV}$ \\
\hline 1 & 1.004 & 226.155 & 666.472 & 0.339 \\
2 & 2.000 & 53.884 & 166.412 & 0.324 \\
3 & 3.004 & 23.13 & 73.082 & 0.316 \\
4 & 4.001 & 12.472 & 40.819 & 0.306 \\
5 & 4.001 & 12.907 & 40.819 & 0.316 \\
6 & 3.004 & 23.059 & 40.819 & 0.316 \\
7 & 2.003 & 53.132 & 165.909 & 0.32 \\
8 & 1.004 & 217.435 & 666.47 & 0.326 \\
\hline
\end{tabular}

To check that the response of the ARDUO matches expectations, the measured photopeak count rates with the background count rate subtracted was plotted in Figure 2.12 as a function of distance from the center of the ARDUO. Given that air attenuation is negligible over these short distances, the measured photopeak count rate should decrease as a function of distance following the inverse square law. The plotted inverse square law prediction was normalized to the average of the measured photopeak count rates at a distance of $1 \mathrm{~m}$ and was overlaid on the plot for comparison with the measured photopeak count rates. The intrinsic photopeak efficiencies were also plotted with the error bars representing the maximum root mean square deviation of the measurements at the same distances. Figure 2.12 shows that the measured photopeak count rates follow the inverse square law as the distance increases. There is no significant trend in the intrinsic photopeak efficiency data. This further indicates that the change in the count rate as a function of distance is accurately defined by the inverse square law. 




Figure 2.12: Plot of the measured photopeak count rates and intrinsic photopeak efficiencies as a function of distance from the center of the ARDUO. The yellow triangles represent the intrinsic photopeak efficiencies and use the right $\mathrm{y}$-axis values. The blue diamonds are the measured photopeak count rates with the background count rate subtracted and use the left y-axis values. The green line is the inverse square law normalized to the average value of the measured photopeak count rates at $1 \mathrm{~m}$.

\subsubsection{Sheet Source Sensitivity}

The sensitivity of the ARDUO to a sheet source was determined through a series of Monte Carlo simulations run by Dr. Andrew McCann. A simulated ARDUO containing the eight crystals and SiPMs within an aluminum housing was placed at altitudes of $5 \mathrm{~m}, 10 \mathrm{~m}$, and $20 \mathrm{~m}$ centered above a $250 \mathrm{~m}$ radius source (Dr. Andrew McCann, written communication, October 17, 2018). This radius was determined to be acceptable for simulating an infinite source, as the ARDUO has a limited field of view based on its altitude. The simulation generated a number of events at an energy of $1.596 \mathrm{MeV}$ that originated from a random location within the source radius. The simulation counted the number of events that were detected by the ARDUO and divided this value by the total number of events that were generated in the $250 \mathrm{~m}$ 
radius source. This ratio is the sensitivity of the ARDUO and is calculated at each of the three altitudes.

The sheet source sensitivity versus the altitude is plotted in Figure 2.13. The simulated data shows how the sheet source sensitivity decreases as a function of the ARDUO altitude. The simulated data was fit with a parameterized inverse square function, shown by the blue dotted line. The function follows the form of,

$$
S=\frac{a}{h^{2}+b}+c
$$

where $S$ is the sheet source sensitivity $\left(\mathrm{cps} /\left(\mathrm{kBq} \cdot \mathrm{m}^{-2}\right)\right), h$ is the altitude above ground level (m), and $a, b$ and $c$ are the fit constants. The fit constants and their statistical uncertainties are shown in Table 2.4 .

Table 2.4: Sensitivity function constants.

\begin{tabular}{ccc}
\hline constant & value & error \\
\hline$a$ & 111 & 13 \\
$b$ & 97 & 11 \\
$c$ & 0.62 & 0.03 \\
\hline
\end{tabular}

The fitted function can be used to find the sensitivity for a source with an energy of $1.596 \mathrm{MeV}$ for an altitude of the ARDUO between $5 \mathrm{~m}$ and $20 \mathrm{~m}$. The sheet source sensitivity function will be used to calculate the surface activity concentration map using the process to be described in Section 3.2.10 for the distributed source trial (Chapter 5). 


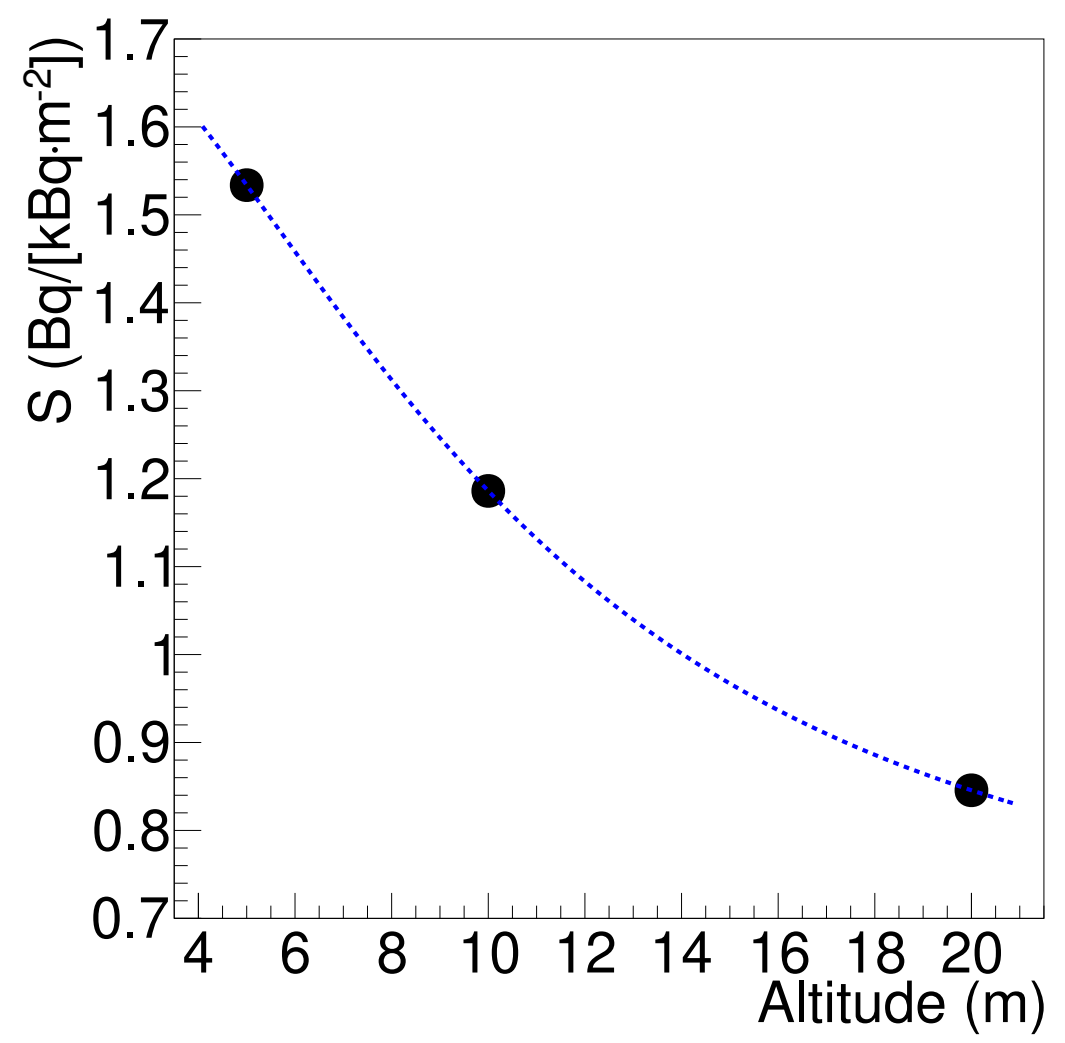

Figure 2.13: Simulated sensitivity to a sheet source as a function of the altitude of the ARDUO for a $1.596 \mathrm{MeV}$ gamma ray source. The error bars on each data point are less than the size of the data point marker. A function was fit to the synthetic data, and is shown by the blue dotted line.

\subsubsection{Direction Reconstruction}

To determine the ARDUO's direction reconstruction capabilities, a series of experiments were conducted in laboratory conditions at the NRCan Geomagnetic Laboratory in the east end of Ottawa. A three-dimensional wooden frame containing three sets of square magnetic field nulling coils was used for source placement [37]. A calibrated point source of ${ }^{137} \mathrm{Cs}$ with an activity at the time of the measurements of $3.2 \pm 0.1 \mathrm{MBq}$ was used.

The configuration of the ARDUO and Responder within the wooden frame is shown in Figure 2.14. Two orientations were used, one where the ARDUO was centered within the whole wooden frame (Figure 2.14a), and one where the ARDUO was centered within one of the square frames (Figure 2.14b). The dimensions of the 
frame had been documented by Robertson [37] and allowed for accurate positioning of the ARDUO and the point sources. No electricity was running through the coils during the experiment. The ARDUO was centered within the wooden frame while mounted on the Responder. A plastic table and plywood board were used to rest the Responder on a set of wooden support beams that passed through the middle of the wooden frame. The point sources were placed at known locations along the inside edge of the wooden frame.

The attenuation of the wooden frame, plastic table and plywood were assumed to have a minor effect on the direction reconstruction. This is because at the chosen source locations, the ARDUO would be near-completely obscured by the structure. This creates an equal reduction in count rate from the attenuation of the impeding structure for all crystals of the ARDUO. As the direction reconstruction algorithm uses the relative count rate in each crystal, a reduction in the total count rate of all the crystals in the ARDUO does not affect its reconstruction capabilities. This setup could not be used for the intrinsic photopeak efficiency calculations as the total count rate measured by the ARDUO is used. The intrinsic photopeak efficiency assumes that the measured count rate is directly resulting from the source, therefore the effect of any material between the source and the ARDUO would result in a false intrinsic photopeak efficiency.

A third set of measurements were taken with the ARDUO centered within the coils without the Responder airframe present (Figure 2.14c). The ARDUO was rested on a foam block to reach the height needed to remain within the center of the square frame.

Additional measurements were taken at distances greater than $1 \mathrm{~m}$ from the ARDUO center. The wooden frame could not be used for these measurements. The second experimental setup shown in Figure 2.15. The ARDUO mounted on the Responder was placed on a plastic table in the middle of the laboratory. The calibrated 


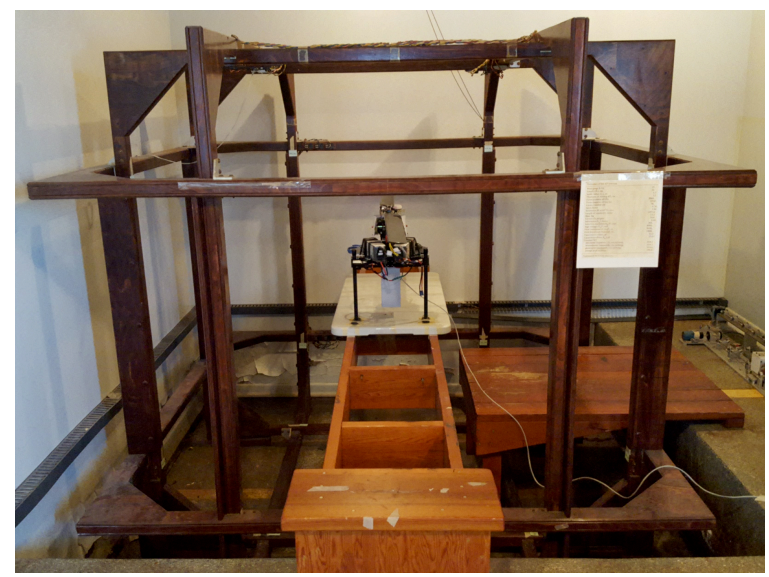

(a) ARDUO mounted on Responder and centered within the whole wooden frame.

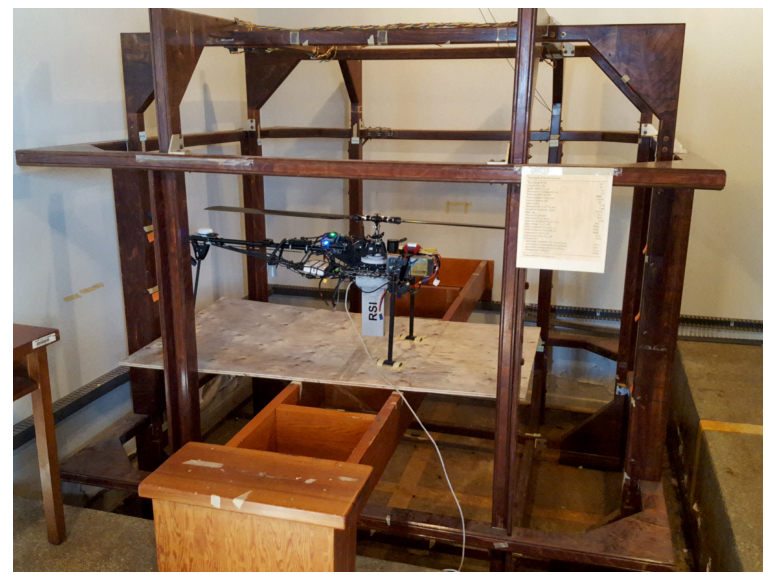

(b) ARDUO mounted on Responder and centered within a single coil.

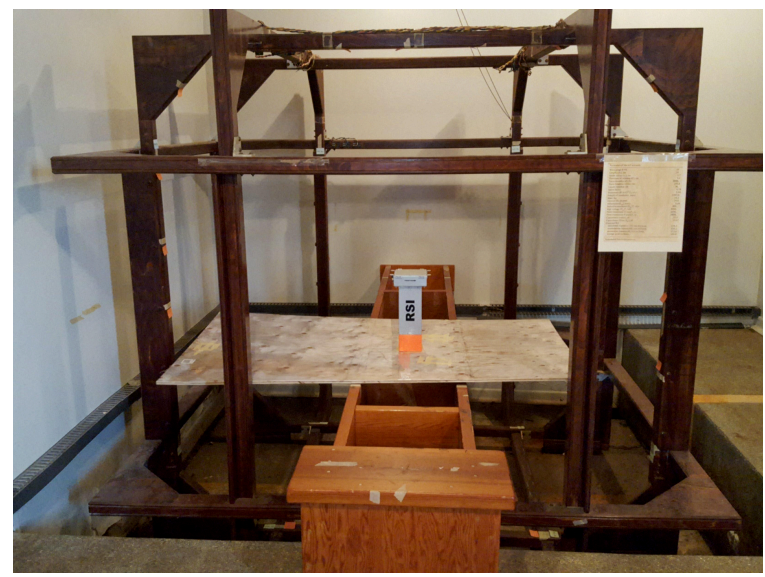

(c) ARDUO centered within a single coil without the Responder airframe present.

Figure 2.14: Laboratory experiment setup with the ARDUO, Responder and wooden frame. The calibrated point source was placed at known locations on the wooden frame. 
source was placed on a wooden table and foam stand at a known distance from the center of the ARDUO. The UAS with the plastic table was rotated around the ARDUO's vertical axis to obtain various azimuth angle measurements. The height of the source was varied to provide changes in the elevation angle.

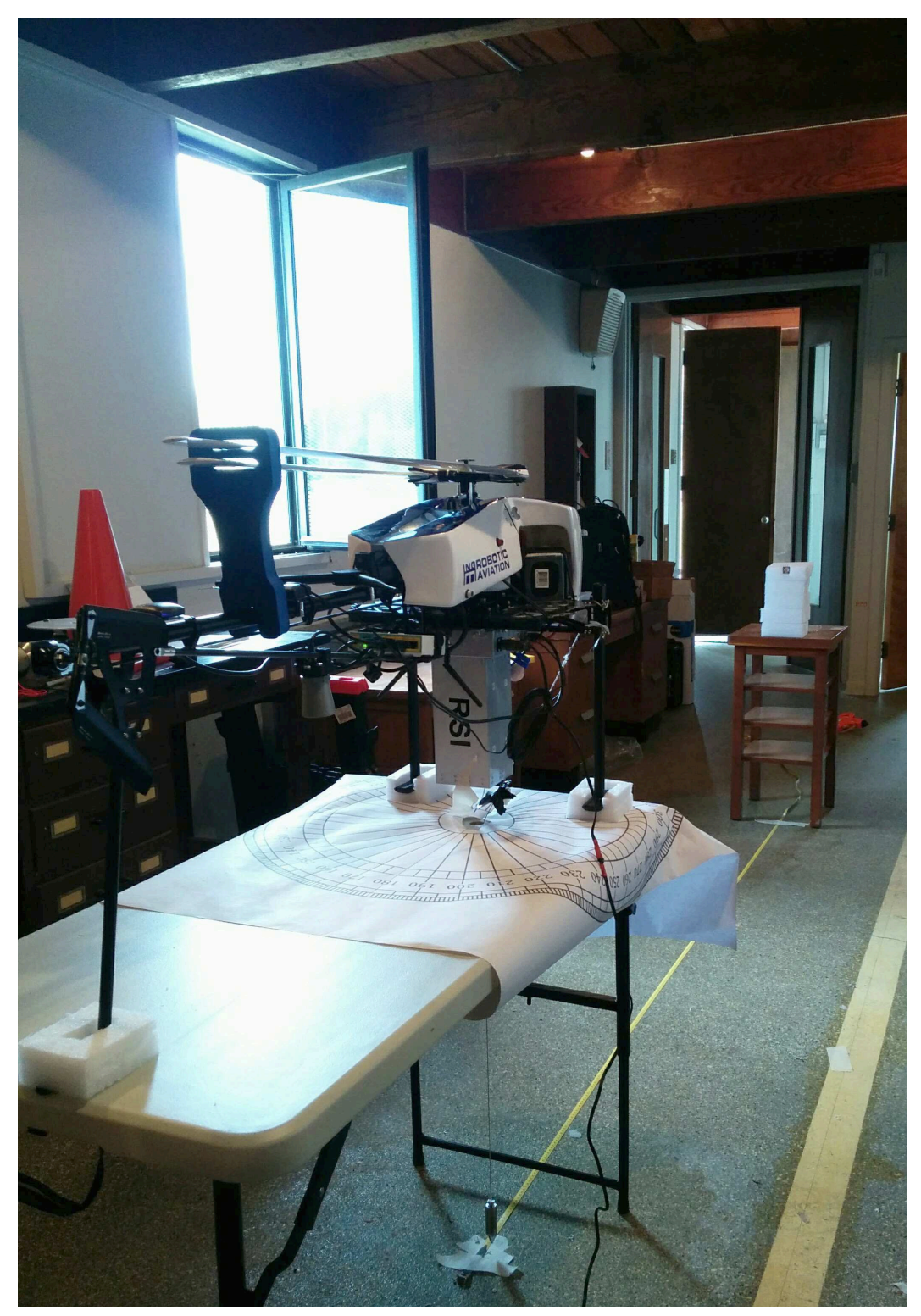

Figure 2.15: The experimental setup for the UAS for direction reconstruction measurements at greater than $1 \mathrm{~m}$ distances. The UAS was placed on a plastic table and the source location was measured from the center of the ARDUO. The source was placed in a foam holder on a wooden table, as seen in the mid-right of the photo. 
The calibrated source locations were chosen at varying azimuth and elevation angles to sample the space around the ARDUO. Distances from the source to the ARDUO ranged from approximately $1 \mathrm{~m}$ to $3 \mathrm{~m}$. A subset of these locations was used for measurements without the Responder airframe present to determine its influence.

To increase the statistical precision of the direction reconstruction, a 5 min measurement was taken at each source location and split into an ensemble of thirty $10 \mathrm{~s}$ datasets. Each $10 \mathrm{~s}$ dataset was used to calculate the count rate in each crystal between an energy window of 0.1 to $3.0 \mathrm{MeV}$. Background measurements lasting $15 \mathrm{~min}$ were collected daily, and the average background count rate in the 0.1 to $3.0 \mathrm{MeV}$ energy window was subtracted from the ten-second dataset count rates in the same energy window. The azimuth and elevation angles were then reconstructed for each dataset in the ensemble.

Following the process laid out in Section 3.2.8 Equation 3.4 to 3.8, the azimuth and elevation angles were reconstructed from each dataset. The mean reconstructed azimuth angle and mean reconstructed elevation angle is calculated from the ensemble of datasets. The root mean square deviation, RMSD, for the ensemble's mean reconstructed azimuth and elevation angles are calculated using

$$
\operatorname{RMSD}=\sqrt{\frac{\sum_{i=1}^{N_{\text {dataset }}}\left(\bar{x}-x_{i}\right)^{2}}{N_{\text {dataset }}}},
$$

where $\bar{x}$ is the mean azimuth (elevation) angle over all datasets, $x_{i}$ is the azimuth (elevation) angle for dataset $i$, and $N_{\text {dataset }}$ is the total number of datasets.

The mean and RMSD of the azimuth angle at each source position is plotted versus the true azimuth angle at elevation angles ranging from $-75^{\circ}$ to $75^{\circ}$ in Figure 2.16a. A one-to-one line is plotted to compare the true and reconstructed azimuth angles. The reconstructed azimuth angles align well with the one-to-one line, indicating that the ARDUO performs well at reconstructing the azimuth angle for a range of elevation angles. Azimuthal reconstructions at elevation angles above $+75^{\circ}$ and below $-75^{\circ}$ were 
not explored as the azimuthal phase space is significantly truncated and vanishes at the poles, where the elevation angle is $\pm 90^{\circ}$.

Figure $2.16 \mathrm{~b}$ shows the mean and RMSD of the elevation angles at each source position compared with the true elevation angles at azimuth angles ranging from $0^{\circ}$ to $330^{\circ}$. The pink diamonds are results without the Responder airframe present. The pink shaded curve represents the simulated ARDUO response as obtained from earlier Monte Carlo design studies [35, 38]. Due to limitations in the geometry of the crystals and their arrangement, little elevation information is available for points at true elevation angles near the horizon. At positive (up) true elevation angles for certain azimuth angles, the airframe appears to have an effect on the elevation reconstruction. The measurement at a true elevation angle of $15^{\circ}$ and true azimuth angle of $180^{\circ}$ appears to be affected by the mass of the tail boom. Likewise, the measurement taken at a true elevation angle of $50^{\circ}$ and a true azimuth angle of $315^{\circ}$ appears to be affected by the mass of the batteries. As the elevation angle approaches vertical, the reconstructed elevation points generally lie near the known system response curve.

For geophysical exploration applications, radiation sources are located on the earth's surface and are therefore located below the ARDUO during flight. As elevation angles are more accurately reconstructed near vertical and the source is assumed for these applications to be in the downwards direction, an elevation cut removing angles above $-40^{\circ}$ will be applied in the direction vector projection analysis described in Section 3.2.9. By removing the poorly reconstructed elevation angles above $-40^{\circ}$, a more accurate analysis of the source location can be conducted.

Nuclear emergency response and environmental applications may have radiation source locations that are not limited to the ground's surface. Sources may be located in buildings or may be airborne in a plume. For these applications, the elevation angle cut would not be used as directional information could be needed to locate 
sources near the horizon or above the ARDUO. As the direction reconstruction is poor for these near-horizon and upwards-pointing elevation angles, future work could be completed to improve the ARDUO direction reconstruction performance for these elevation angles. 


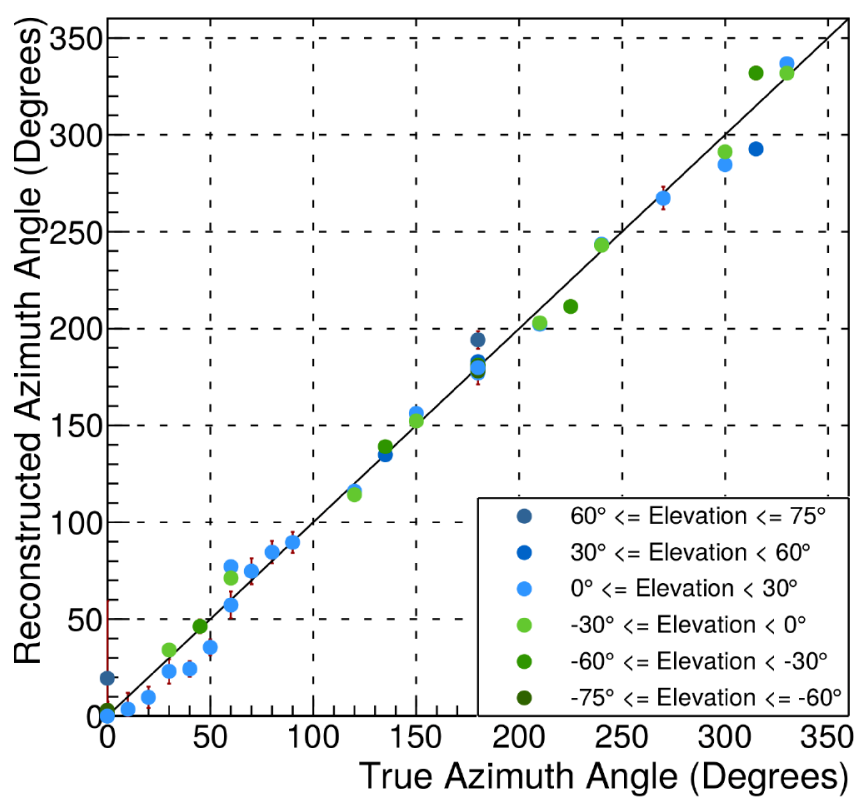

(a) Reconstructed azimuth angles.

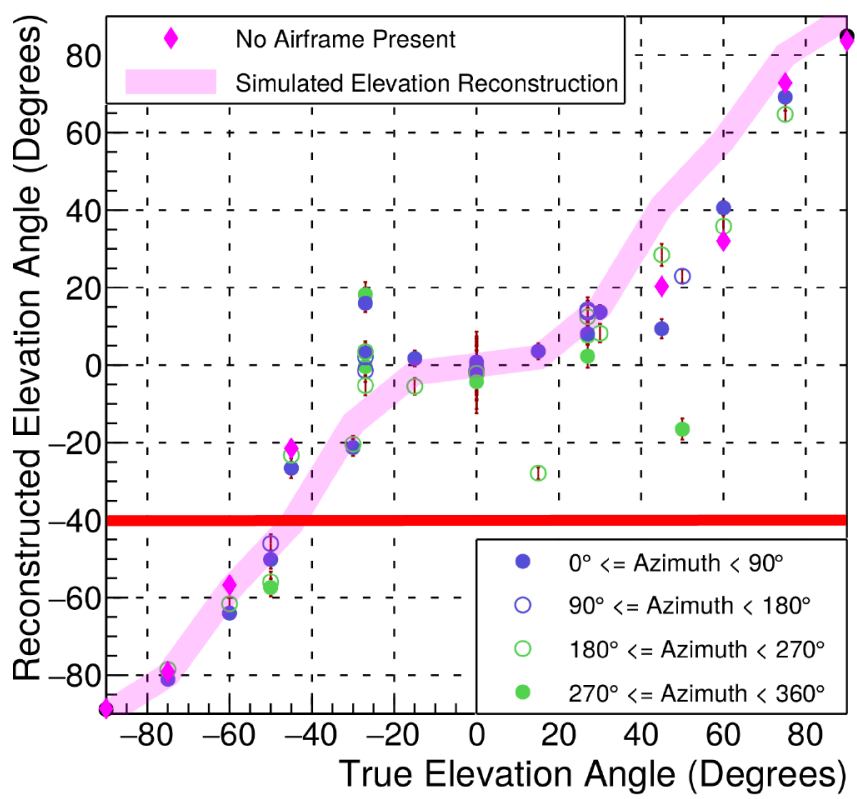

(b) Reconstructed elevation angles.

Figure 2.16: Results from the laboratory direction reconstruction tests. Each data point represents the mean value of the reconstructed direction vector angles from thirty $10 \mathrm{~s}$ datasets. The error bars show the root mean square deviation of the 10 s measurements. a) Reconstructed azimuth angle versus true azimuth angle for a variety of elevation angles. All measurements were taken with the airframe present. The measurements are coloured based on their elevation angle using $30^{\circ}$ increments up to an elevation angle of $\pm 75^{\circ}$. b) Reconstructed elevation angle versus true elevation angle for a variety of azimuth angles. The pink shaded curve is the ARDUO response estimated from Monte Carlo studies. The pink diamonds are measurements taken without the airframe. Each measurement is coloured based on the azimuth quadrant their respective azimuth angle lies within. The solid red line shows the $-40^{\circ}$ elevation angle cutoff used in the direction vector projection analysis. 


\section{Chapter 3}

\section{Methods}

In this chapter, the data acquisition and data processing methods used to conduct and analyze the surveys with NRCan's radiometric UAS are presented. The acquisition methods are similar to those used for manned aerial radiometric surveying, with modifications to account for the UAS's flight capabilities. The processing methods are also based on the methods used to process the data from manned aerial radiometric surveys. There are additional steps in the processing methods to incorporate the UAS autopilot data as well as for processing the direction information from the ARDUO.

\subsection{Data Acquisition Methods}

The data acquisition methods are presented in this section. The process starts with the creation of a flight plan. This can be done in advance of surveying, using the information available about the surveying site and the potential radioactive target. At the surveying site, the UAS goes through a series of pre-flight checks and preparations to determine if it is ready for flight. The sensor systems are monitored during flight

to determine the health of the UAS as data is being collected. After the flight, a series of operations is carried out to collect all the data. 


\subsubsection{Flight Planning}

The flight plan of the UAS is a series of instructions that the UAS follows in a near-autonomous flight mode. The flight plan is created using the Mission Planner software [11] on the GCS laptop. The flight plan is typically composed of a 'Takeoff' command that tells the UAS to lift off the ground to a given altitude, a series of altitude- and position-marked waypoints typically laid out in a grid pattern that the UAS follows, and then a 'Return to Launch' command which tells the UAS to return to the takeoff location and land. Additional commands can be used to change the speed of the UAS during the flight or have the UAS hold its position for a given amount of time.

Figure 3.1 shows the mission plan in the Mission Planner software that was used for the distributed source trials. The center of the screen shows the map with the flight plan overlaid. The waypoints are marked with the green bubbles and are numbered according to their flight order. They are connected with yellow lines that indicate the flight path, and the yellow arrows mark the flight direction. The green ' $\mathrm{H}$ ' bubble indicates the takeoff and landing, or home, location. The UAS would takeoff from the home location, fly to waypoint 2 , follow the flight plan line to waypoint 3 , and would continue to fly along the yellow flight plan line to each waypoint in numbered order. At the end, in this case waypoint 41, the UAS would 'Return to Launch', therefore returning to the home location and landing.

The flight plan is often determined prior to surveying. A desktop study of the area of interest is typically completed to obtain information about the radioactive target, the terrain, the vegetation, potential takeoff and landing locations, UAS flight regulations in the area and the expected weather conditions in the area. This can involve examining aerial images, historical information, previously completed research, and previously conducted survey maps of the area. Site visits can also be conducted 


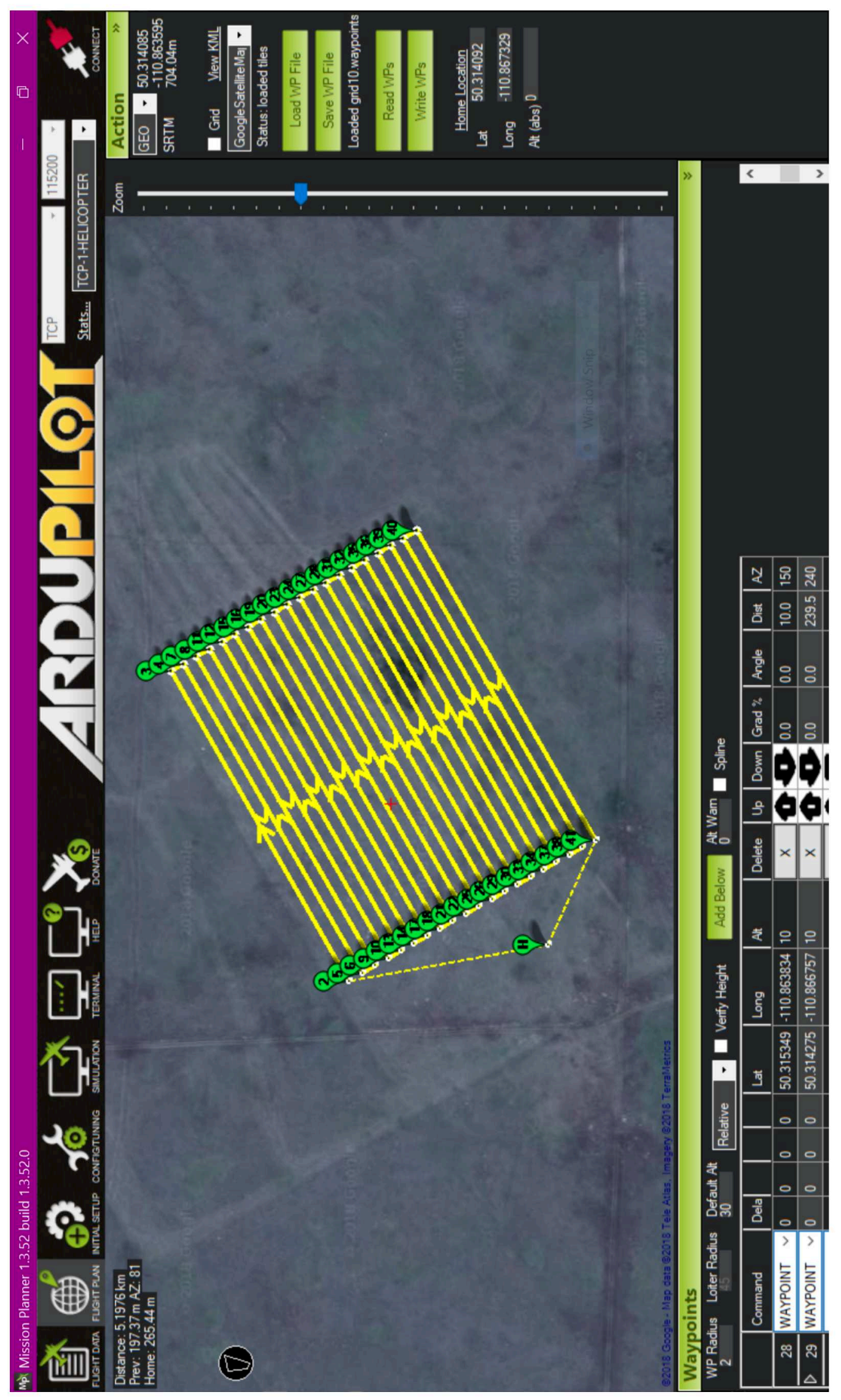



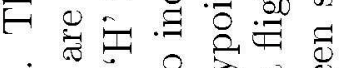

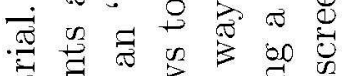

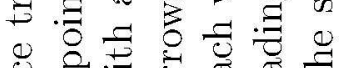

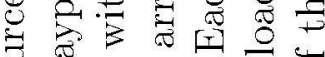



중

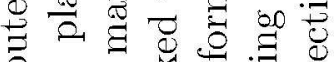

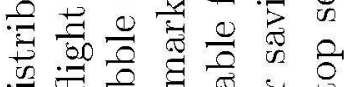

-

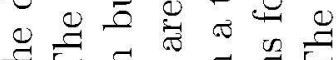

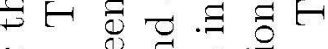

ธ્વ

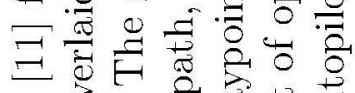

० ठ․

$\Rightarrow+\dot{0}=\pi$

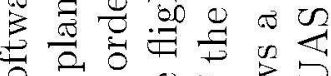

政

क .

当

क

$\exists \rightrightarrows \Xi \omega^{2}$

운 원

苟

ه

मे $8=0$ क

so 0 \%

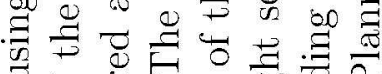

ठ

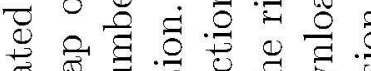

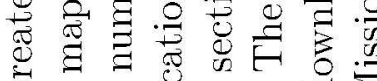

¿

สี

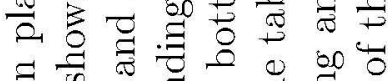

후 का

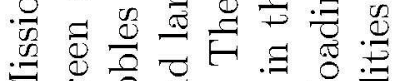

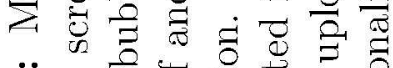

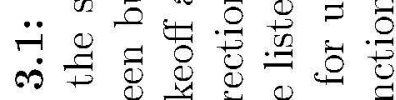

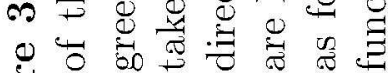

$\exists$

$\stackrel{50}{5}$ 
prior to surveying to obtain current information about the survey area and confirm the information obtained through the desktop study. The information on the area of interest can then be used to guide the flight planning process.

A total survey area as well as desired spatial resolution can be determined from the information about the area of interest and the radioactive target. These aspects will be dependent on the objectives of the survey. For example, a reconnaissance survey will have a lower spatial resolution with a larger total survey area, or a detailed survey to capture the smaller features of the radioactive structure of interest can have a high spatial resolution and cover a smaller area. The cost of the survey can also affect the total survey area and spatial resolution, as higher spatial resolution surveys and larger survey areas will take more time to complete and therefore increase costs.

Once the total survey area is known, the ideal flight plan altitude (m), line spacing $(\mathrm{m})$, and speed $(\mathrm{m} / \mathrm{s})$ can be determined to obtain the desired spatial resolution. The altitude determines the ground sampling area, or field of view, of the ARDUO. A lower altitude will reduce this area, therefore increasing the spatial resolution. Lower altitudes also reduce the coverage of the survey area, therefore tighter line spacing and slower speeds are needed to achieve the same ground coverage as higher altitude surveys. The flight speed determines the spatial resolution and ground coverage in the flight direction.

The flight speed is determined using the data sampling rate. For the ARDUO data is collected once per second. The speed therefore determines the distance between each data point, as well as the ground area sampled for each data point accumulation period.

The line spacing affects the spatial resolution and ground coverage in the direction perpendicular to the flight direction. Typically, line spacings are larger than the 
distance traveled during each data point accumulation. This creates a larger spatial resolution in the direction perpendicular to the flight line, and a smaller spatial resolution along the flight line.

For geophysical exploration, flight lines are typically oriented perpendicular to the strike of the structure of interest. This results in a higher spatial resolution across the strike of the structure, where there tend to be larger variations in the structure of interest over shorter distances.

After determining the ideal flight parameters, changes to the flight plan may be necessary to account for variations in terrain, the presence of trees, the presence of tall man-made structures, the takeoff and landing locations in the area, the number of flights required to cover the total survey area, and the UAS flight regulations in the area.

The variations in terrain, and the presence of trees and tall man-made structures can affect the minimum altitude at which the UAS can safely fly. This then affects the minimum spatial resolution that can be achieved by the UAS. Additionally, a higher altitude results in a lower sensitivity of the ARDUO to the radiation on the ground. This may result in the need for longer accumulation times in one position to obtain a more statistically significant sample.

The takeoff and landing locations can affect the flight plan. A level area clear of tall vegetation is typically required for UAS operations, with the size of the area dependent on the UAS being used. The NRCan UAS requires a clear, level area with a $4 \mathrm{~m}$ radius for takeoff and landing [8]. Additional space is also needed for the GCS. A survey area may be limited in takeoff and landing locations, therefore limiting the distance the survey area can be from the takeoff and landing location. As a survey becomes further from the takeoff and landing location, additional time is needed for the UAS to travel to the survey area and back. The NRCan UAS flight time is approximately $20 \mathrm{~min}$, therefore the time taken to travel to the survey area 
should be minimized to allow the UAS to spend more time completing the survey grid.

Additionally, the limited flight time of the UAS may require a number of flights, and therefore flight plans, to cover the total survey area. This will depend on the flight speed and line spacing, as slower flights with tighter line spacings will cover less area and take more time to complete. If the total survey area is too large to complete in one survey with the necessary flight parameters to achieve the spatial resolution needed, multiple flights can be conducted to cover the total survey area. The total survey area would be broken into a number of sections, each corresponding to an individual survey flight. The data from each survey flight can then be amalgamated in post-acquisition process to produce a map for the total survey area. The addition of multiple flights will significantly increase the total time taken to survey the area, as pre-flight and post-flight operations will add additional time between each flight. For NRCan's radiometric UAS, six 20 min flights are typically achieved in one day. Multiple flights can also add complexity to the data processing as there can be larger variations in the sensor calibrations, drifts and errors between each flight than within a single flight. Additional steps, such as tie lines, may be required for data quality assurance, therefore also increasing the total surveying time and costs.

UAS regulations can also affect the flight plan. Currently in Canada, the use of a UAS for non-recreational purposes requires the operator to obtain a Special Flight Operations Certificate, or operate under a set of exemption rules set by Transport Canada [39]. Additionally, a UAS can only be flown if it is within visual line of sight of the operator. This can affect operations in areas with tall trees that may obscure the UAS as it flies further from the takeoff and landing site. To increase the operator's visual line of sight in these situations, scaffolding or an elevated platform can be used. The operator would then be elevated to see over the tree canopy. This 
may not be possible in area where there is limited vehicle access to the site, or in remote areas where elevated platforms may not be available.

\subsubsection{Pre-flight Operations}

The GCS equipment is set up at the launch site with the mission planning laptop, radiation data laptop and all telemetry systems turned on. The batteries that are used to power the Responder airframe can be charged in advance of surveying, or could be set up for charging upon arriving at the launch site.

The Responder airframe with the ARDUO mounted underneath is placed at its takeoff location. A charged pair of batteries is attached to the airframe using several elastic bands. The batteries are connected to the electrical systems, powering the Responder airframe's motors, telemetry systems, sensor systems, autopilot, and the ARDUO. The airframe is inspected for mechanical and electrical damage to determine if the system is airworthy. The GCS mission planning laptop running the Mission Planner software [11] is connected to the Responder's autopilot through the telemetry systems.

Visual checks of the flight data in the Mission Planner software are completed to determine if the sensors and autopilot systems are producing sensible data that can be used for flight operations. The Mission Planner flight data real-time display is shown in Figure 3.2. While the display is shown during the flight over the distributed source, the same display and data are checked for sensibility prior to flight. The flight data display is further explained in Section 3.1.3. The UAS data that is checked prior to flight includes the orientation, the battery voltage and current, the telemetry connection health, the GNSS position and quality, and the altitude of the airframe. For the orientation checks, the airframe is partially rotated around its three axes and the response in the real-time display is visually checked to determine if the displayed response corresponds well to the rotation. The Mission Planner software also provide 
alerts if the compass, Global Positioning System (GPS) or EKF are not functioning correctly. If there is an alert, the Mission Planner software also prevents the UAS from taking off. When the issue is resolved, the alert is stopped.

The autopilot parameters and fail-safes are checked and changed if needed. This includes the actions the autopilot will take if the battery voltage becomes too low, if the UAS reaches the survey zone limit defined by a geofence, the flight speeds, and actions to take if any of the three telemetry signals loses connection. The flight plan is also uploaded to the autopilot and checked for any errors.

The Safety Pilot checks the radio control transmitter for mechanical damage. $\mathrm{He} / \mathrm{She}$ checks that the control switches result in the correct response in the Responder airframe's motor systems controlling the rotor blades. All switches are set to off prior to flight.

The ARDUO is powered through the Responder airframe's batteries. These are connected at the beginning of the equipment setup and checks, so that the internal temperature can stabilize, and therefore reduce the amount of gain drift in the ARDUO. The ARDUO is connected to the radiation laptop through the telemetry systems. The RadAssist software from Radiation Solutions Inc. [5] is used. The real time data stream of the ARDUO is viewed in real time, and checked to determine the telemetry connection is working. After all the safety checks are complete the gain is calibrated using two check sources of ${ }^{137} \mathrm{Cs}$. The check sources are removed prior to takeoff. The RadAssist software is set to record the ARDUO data prior to takeoff.

The autopilot automatically logs all its positioning, orientation, and input sensor data on-board on a microSD card.

The GCS operator and Safety Pilot review the flight plan and emergency response procedures, and then follow the takeoff procedures. As a safety precaution the UAS requires the system to be pre-armed to engage the rotor control motors, and armed to allow for the main motor that controls the rotor blade rotation to function. The 
Safety Pilot is responsible for arming the system, and is responsible for turning on the main motor using a throttle switch. Once the main motor is turned on, the main rotor is given a period of approximately $12 \mathrm{~s}$ for it to reach full speed. The Safety Pilot then changes the flight mode to 'Auto', which causes the UAS to takeoff and follow the loaded flight plan.

\subsubsection{In-flight Monitoring}

During flight, the Mission Planner display on the GCS laptop is monitored by the GCS operator to check the health of the flight systems. Figure 3.2 shows the realtime Mission Planner display during the distributed source trials. The display shows contains three sections. One section shows the roll, pitch and altitude information, as well as information on the battery, telemetry, EKF, autopilot vibration, and GPS health. Another section of the display contains the map view with the flight plan overlaid. The flight plan overlay is displayed using the same features as in the flight planning display shown in Figure 3.1. The GNSS position quality is shown in the bottom left corner of the map display. The map display also shows the location of the UAS as a helicopter icon with the nose of the helicopter icon indicating the UAS heading. The purple line shows where the UAS has already flown. For this screenshot, the UAS has completed flying two flight lines, and has turned around to start the third line. The last section contains the GCS actions. These actions can be executed by the GCS operator during the UAS flight. For example, if the flight needs to be aborted, the GCS operator can execute the 'RTL', or 'Return to Launch', command to have the UAS abort the current flight plan, return to the takeoff location and land.

The quality of the radiation data during the flight is also examined by the GCS operator, or additional visual observer, using the RadAssist display on the radiation 


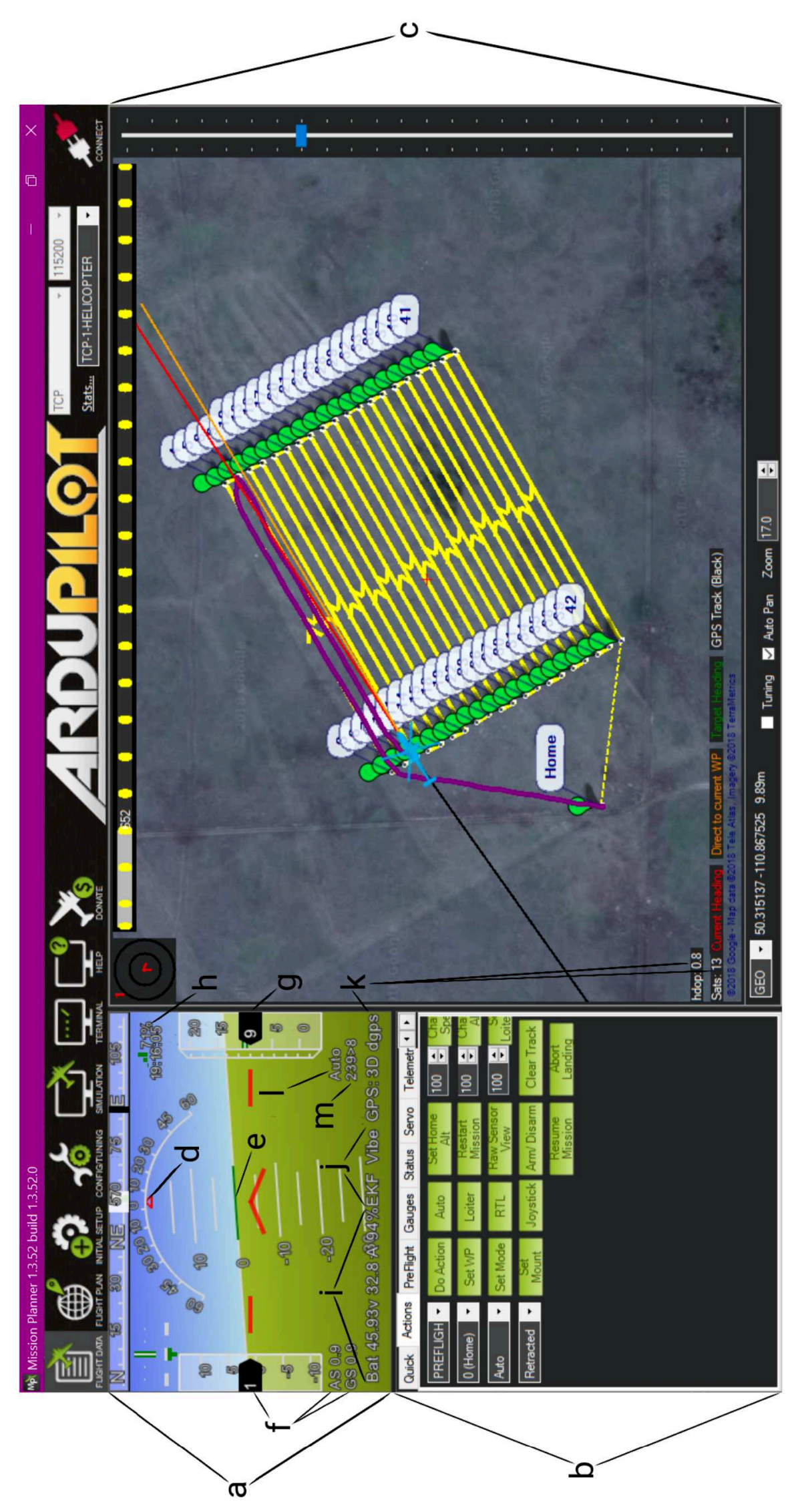

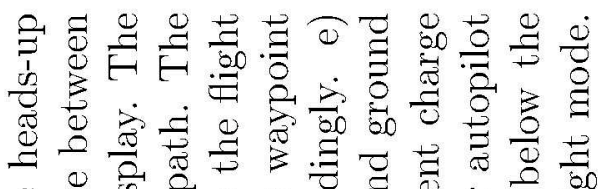

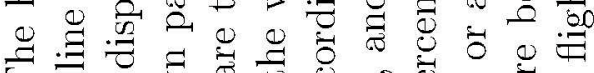

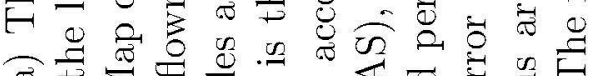

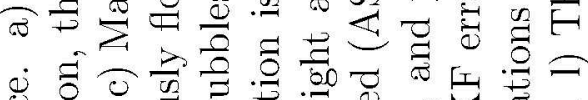

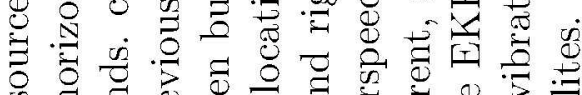

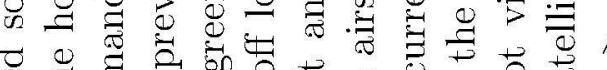

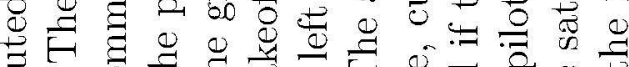

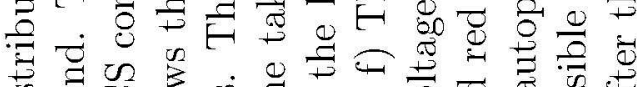

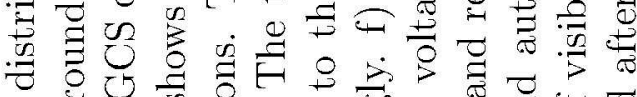
क्ष० की

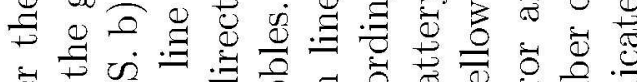

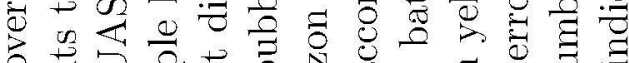

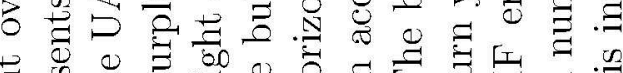

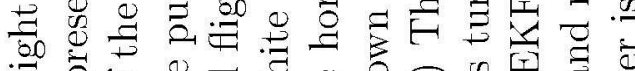

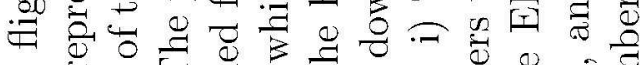

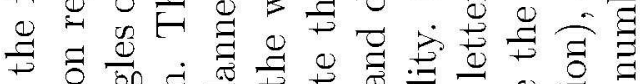

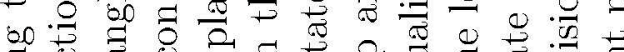

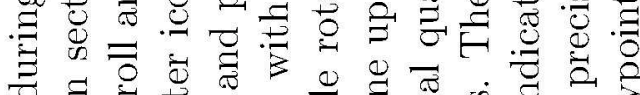
व

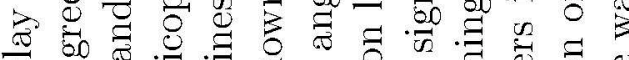

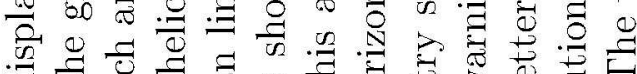

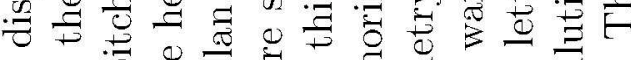

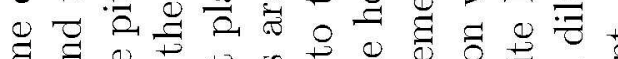
$\exists \widetilde{\Xi}$

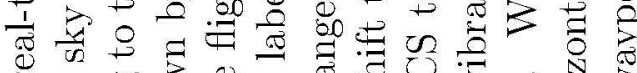

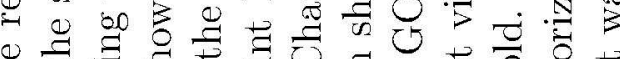

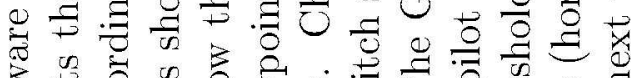

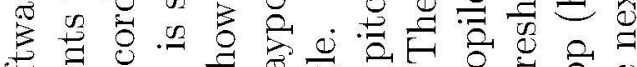

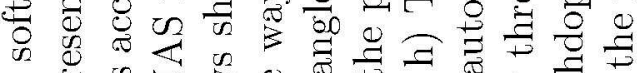

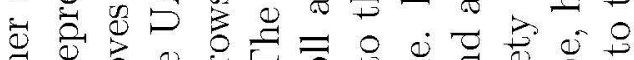

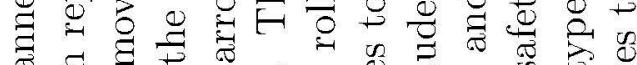

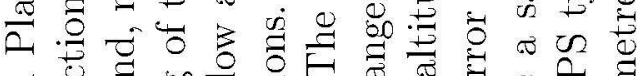
亏

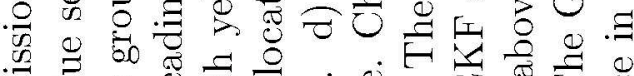

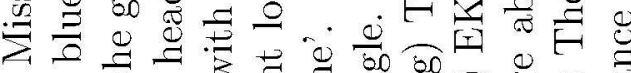

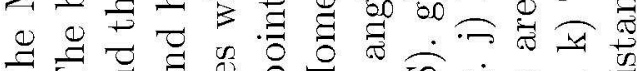
F

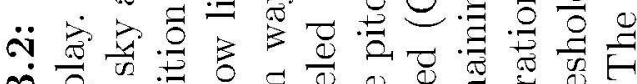

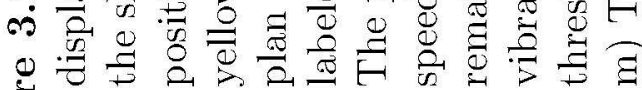

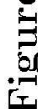


data laptop (shown in Figure 2.6). The Safety Pilot keeps within visual line of sight of the UAS, and observes the UAS as it is flying.

If any unwanted signs are exhibited by the Mission Planner display, RadAssist display, or are shown by the UAS in flight, the flight can be aborted by the Safety Pilot. Should this occur, the Safety Pilot can change the flight mode of the UAS from 'Auto' to a semi-manual mode to maneuver the UAS. Typically, the Safety Pilot holds the UAS to the position at which the flight plan was aborted, while a 'Return to Launch' command is issued by the GCS operator using the GCS laptop. The UAS will then return to the takeoff location and land. The Safety Pilot could also land the UAS in the semi-manual flight mode.

\subsubsection{Post-flight Operations}

After the flight is conducted, the data from the ARDUO is set to stop recording. An initial processing step is completed in the RadAssist software to select only the data collected from the survey grid pattern and convert the raw data file to a file that is usable in post-processing.

The autopilot data is taken from the on-board microSD card. An initial processing step is completed in the Mission Planner software to convert the raw data file into a readable comma-separated values file.

The UAS can be used for additional flights following the pre-flight procedures. If no additional flights are needed, the UAS airframe and GCS are powered down, and packed for transport.

All flights are logged in a logbook. The weather conditions for each flight are recorded along with the flight time, the takeoff and landing times and the battery voltage at takeoff and landing. The total flight times for the UAS are added and recorded. Maintenance of the UAS is required periodically based on the total flight time of the system. 


\subsection{Data Processing Methods}

This section presents the general methods used to process the data from the ARDUO and the UAS autopilot. A summary of the processing sequence is shown in Figure 3.3. The process begins with analyzing and correcting the radiation data. Then the radiation data is integrated with the Responder's autopilot data. Two maps are produced, a map of the photopeak count rate with the direction vectors overlaid, and a map of the direction vector intersection points with the ground. Finally, source strength estimations are conducted for the point source trial as well as for the distributed source trial. The details of each processing step are explained in the following subsections.

\subsubsection{Count Rate Determination}

Count rates are determined using two energy windows: a total-count energy window and an isotope-dependent energy window. The total-count window ranges from 0.1 to $3.0 \mathrm{MeV}$. The restriction to an energy above $0.1 \mathrm{MeV}$ removes the possible effect of low energy noise from the ARDUO's electronics. The restriction to energies below 3.0 MeV suppresses the contribution of energy deposits due to cosmic rays. The total-count window is used for the direction reconstruction process described in Section 3.2.8. The isotope-dependent energy window is determined by examining the spectra from the survey for the presence of any photopeaks. From the photopeaks, an isotope can be identified and the energy window is established using a range of 3 standard deviations around the isotope's photopeak of interest. The count rate in the isotope-dependent energy window is used for the point source and distributed source strength estimations. 


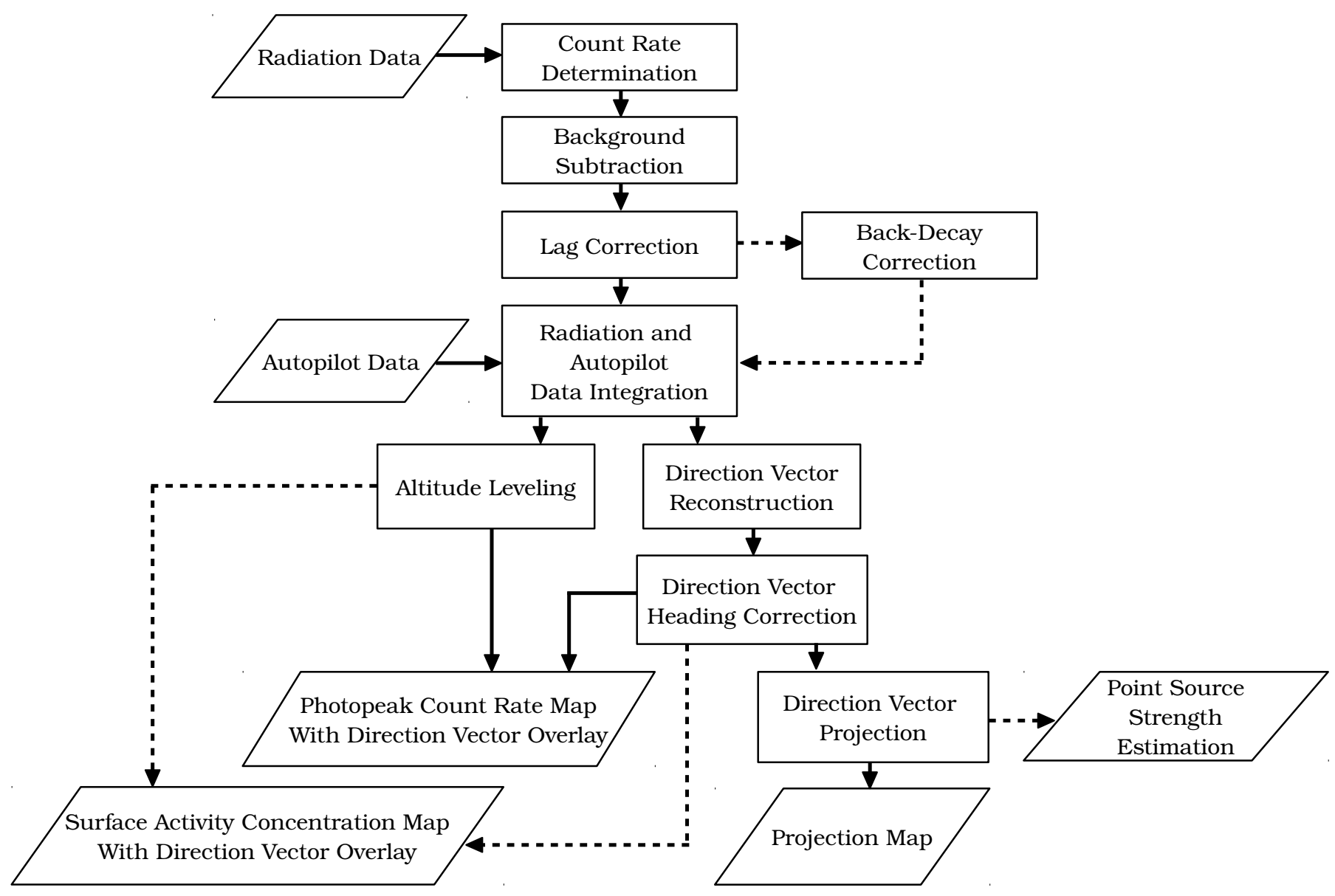

Figure 3.3: Illustration of the data processing sequence. Inputs are the radiation data from the ARDUO and the position and orientation information from the Responder autopilot. Optional analyses are indicated by the dashed lines. The main outputs are two maps; the first is a map of the photopeak count rate with an overlay of the direction vectors, and the second is a map of the intersection points between the ground surface and the direction vectors. The optional outputs are a point source strength estimation, and a surface activity concentration map with the overlaid direction vectors.

\subsubsection{Background Subtraction}

When conducting a geophysical exploration survey, the desired signal is being caused by ${ }^{40} \mathrm{~K},{ }^{238} \mathrm{U}$, and ${ }^{232} \mathrm{Th}$, and background is expected from atmospheric radon, cosmic sources, and the aircraft, including on-board equipment and occupants. Methods to remove the background signal from survey data for the three geological targets are done by collecting data over water at varying heights to determine the cosmic effects, 
and by using an upward-looking spectrometer. The methods are further explained in the report by the International Atomic Energy Agency [7].

For the experiments conducted in this thesis, the background signal is determined by conducting a survey over the area prior to source placement. The dominant background is caused by the natural sources of ${ }^{40} \mathrm{~K},{ }^{238} \mathrm{U}$, and ${ }^{232} \mathrm{Th}$, with smaller contributions from atmospheric radon, cosmic sources and the aircraft. The average background count rate for the area in the total-count and isotope-dependent energy windows are subtracted from each data point count rate in total-count and isotopedependent energy windows for the surveys over the sources.

\subsubsection{Lag Correction}

The ARDUO collects data at a rate of one sample per second. As the UAS surveys at speeds upwards of $2 \mathrm{~m} / \mathrm{s}$, the UAS covers a significant distance during the one-second data accumulation period. This distance is accounted for by applying a lag correction, which averages the position of the ARDUO to a mid-accumulation point, as shown in Figure 3.4. As the data timestamp occurs at the beginning of the accumulation period, $0.5 \mathrm{~s}$ is added to each timestamp. The position of the ARDUO at the midaccumulation point is determined using the data collected by the ARDUO autopilot, and is explained in further detail in Section 3.2.5.

\subsubsection{Back-decay Correction}

An additional step can be applied to normalize the count rate attributed to the source at the time of the survey to a known count rate of the source at a reference time. This step is known at the back-decay correction, as the radioactive decay between the reference time and the survey time is accounted for. This processing step is only used if the activity of the source known at a certain time. The back-decay correction 


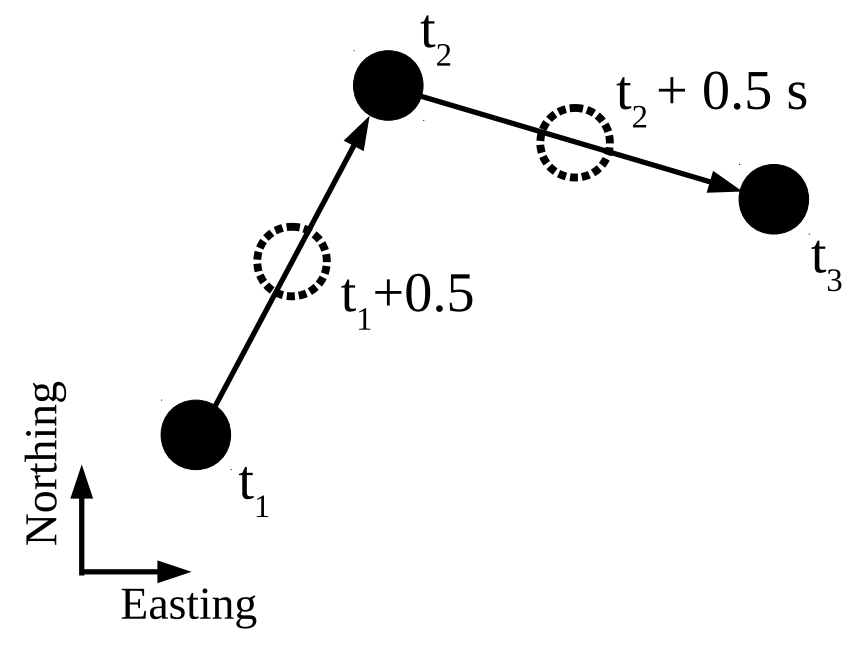

Figure 3.4: Diagram of the application of a lag correction. As each data point is timestamped at the beginning of the data accumulation period of $1 \mathrm{~s}, 0.5 \mathrm{~s}$ is added to each timestamp.

is typically completed for sources with short half-lives as the amount of decay over the survey time can have a significant effect on the measured count rate. This allows for consistency in the count rate throughout the survey, as well as for a potential comparison with other surveys and instrument measurements that were conducted using the same source at different time periods. The background-subtracted count rate for each data point is converted to a count rate at the reference time using the half-life of the isotope and the lag-corrected time of each data point measurement. This is completed using

$$
n_{\mathrm{c}, i}=\frac{n_{i}}{e^{-\frac{\ln (2)}{t_{1 / 2}}\left(t_{i}-t_{0}\right)}},
$$

where the half-life of isotope is $t_{1 / 2}(\mathrm{~s})$, the lag-corrected time for data point $i$ is $t_{i}$ (s), the reference time is $t_{0}(\mathrm{~s})$, the measured count rate at data point $i$ is $n_{i}(\mathrm{cps})$, and the back-decayed count rate at data point $i$ is $n_{\mathrm{c}, i}(\mathrm{cps})$. 


\subsubsection{Radiation and Autopilot Data Integration}

The UAS's PixHawk 1 autopilot determines its latitude, longitude, altitude above ground level, and yaw using an extended Kalman filter and the suite of sensors listed in Chapter 2. This is logged every $0.02 \mathrm{~s}$. The autopilot data stream contains more accurate positioning information than the ARDUO's GNSS receiver, and collects orientation information that the ARDUO does not measure. To merge the autopilot and ARDUO data streams, the lag-corrected timestamps from the ARDUO are used to relate the radiation measurements with the autopilot's position and attitude measurements. The merged data stream - containing the ARDUO's lag-corrected timestamps and radiation data, and the autopilot's position and orientation data - is used in the following processing steps.

\subsubsection{Altitude Leveling}

During the survey, the Responder's altitude can vary due to flight conditions. Variation in flight altitude could cause changes in the count rate, as this would change the distance from the source to the ARDUO. Increases in altitude would decrease the measured count rate, therefore causing an apparent reduction of the signal from the source. Similarly, a decrease in altitude would cause an increase in the measured count rate, leading to an apparent increase in the signal from the source. The effect on the count rate due to changes in altitude therefore needs to be examined.

For geological sources which are effectively infinite in extent, the count rate as a function of altitude can be approximated with an exponential function [7]. For the energy windows of the geological sources of ${ }^{238} \mathrm{U},{ }^{232} \mathrm{Th}$, and ${ }^{40} \mathrm{~K}$, the altitude corrected count rate, $n_{\mathrm{h}}$ (cps), has been estimated using,

$$
n_{\mathrm{h}}=n e^{-\mu_{\mathrm{h}} h},
$$


where $\mu_{\mathrm{h}}$ is the energy window height attenuation coefficient $\left(\mathrm{m}^{-1}\right), n$ is the observed count rate (cps), and $h$ is the altitude above ground level (m) [7]. The energy window height attenuation coefficient is determined using a calibration range. The calibration range is $8 \mathrm{~km}$ long area with uniform concentrations of ${ }^{238} \mathrm{U},{ }^{232} \mathrm{Th}$ and ${ }^{40} \mathrm{~K}[6]$. Flights at a range of altitudes are conducted over the calibration range. The height attenuation coefficient is estimated from an exponential fit to the observed count rate versus the altitude. The spectrometer's altitude above ground level can vary with topography, therefore a digital elevation model for the survey area can be used to account for the topographic changes.

The sources used for the research described in this thesis were limited in their extent, therefore the typical altitude leveling methods described in the previous paragraph cannot be used. Moreover, the previously described methods would require a test strip containing a uniform concentration of the isotopes used for this research, ${ }^{137} \mathrm{Cs}$ and ${ }^{140} \mathrm{La}$, in order to determine the sheet source sensitivity (see example from Sanada et al. [23]). Finding or making a test strip for these isotopes is not feasible for this thesis.

For non-infinite sources, such as those used in this thesis, the altitude correction would vary over the survey area and would depend upon the horizontal distance between the source and the spectrometer. To estimate the maximum effect on the count rate from changes in altitude, the mean altitude and its standard deviation for the survey area are calculated. Assuming a point source is within the survey area, the maximum change in the count rate with respect to the average count rate due to a difference in altitude would be at a location directly above the source. In this case, the count rate altitude correction factor, $c_{\mathrm{h}}$, is given by,

$$
c_{\mathrm{h}}=\left(\frac{h_{i}}{h_{0}}\right)^{2} e^{-\mu_{\mathrm{air}}(E)\left(h_{0}-h_{i}\right)}
$$


where $h_{0}$ is the nominal altitude of the survey $(\mathrm{m}), h_{i}(\mathrm{~m})$ is the altitude at the data point $i$, and $\mu_{\text {air }}$ is the attenuation coefficient of air at energy $E\left(\mathrm{~m}^{-1}\right)$.

A $\pm 10 \%\left(0.9 \leq c_{\mathrm{h}} \geq 1.1\right)$ variation in the count rate caused by altitude changes, was determined to be an acceptable range for the experiments conducted in this research. The altitude used in the survey is from the UAS autopilot. This altitude is offset by $0.3 \mathrm{~m}$ from the center of the ARDUO where the direction reconstruction, intrinsic photopeak efficiency and sheet source sensitivity are calculated from. The offset of $0.3 \mathrm{~m}$ is therefore subtracted from the autopilot altitude for all subsequent calculations to be done using an altitude relative to the center of the ARDUO. For a nominal flight at an altitude of $10 \mathrm{~m}$, the altitude used in both the point source and distributed source trials, the center of the ARDUO would be at a nominal altitude of $9.7 \mathrm{~m}$. Using a nominal altitude of $9.7 \mathrm{~m}$, the resulting altitude range for a $10 \%$ variation in the count rate is $9.2 \mathrm{~m}$ to $10.2 \mathrm{~m}$. This is a range of $5 \%$ around the nominal altitude. The nominal altitude can vary from the actual survey flight altitude as the UAS adjusts to wind conditions. To account for this variation, a new altitude range is calculated from the average survey altitude. As the nominal altitude and the average survey altitude are similar, the same $5 \%$ range around the nominal altitude is used to calculate the range around the average survey altitude. Data outside of the altitude range centered on the average altitude is excluded from the rest of the analysis.

While the acceptable altitude range accounts for the stochastic fluctuations in the survey altitude, there may still be a systematic offset in the altitude measurement from the autopilot. This may be caused by uncertainties in the barometric pressure and GNSS altitude data that are used in the EKF to determine the autopilot altitude. From visual observations, the systematic error in the altitude may be up to $1 \mathrm{~m}$. This systematic error is considered for the error in the point and distributed source strength estimation errors. 


\subsubsection{Photopeak Count Rate Mapping}

With all preceding corrections made, the latitude and longitude from the autopilot are converted from the geographic World Geodetic System 1984 latitude and longitude system to easting and northing coordinates in the projected Universal Transverse Mercator (UTM) system in the corresponding zone of the survey using the GeographicLib $\mathrm{C}++$ library [40]. Each data point is mapped based on its location. The photopeak count rate in cps for each data point is interpolated using the inverse distance weighted (IDW) method [41]. The interpolated photopeak count rate map can be used to interpret the spatial distribution of the radiation in the survey area.

\subsubsection{Direction Reconstruction}

The direction vector pointing towards a detected radiation source is calculated using the self-shielding configuration of the crystals. The crystals with relatively higher gamma count rates will be closer to the source, while the crystals behind will have relatively lower gamma count rates due to shielding from the front crystals. The relative count rates in each of the eight crystals over an energy range of 0.1 to $3.0 \mathrm{MeV}$ are used for the calculation. Use of the total-count energy window as opposed to an isotope-dependent photopeak window increases the signal to noise ratio as it includes the partial gamma-ray energy deposits that are part of the source's signal. A direction vector in three dimensions, $\mathbf{v}=(u, v, w)$, is calculated according to Equation 3.4 to 3.6, where $n_{\text {crys }, i}$ is the number of counts per second in crystal number $i$. The crystal numbering scheme was presented in Figure 2.5b.

$$
\begin{aligned}
& u=n_{\mathrm{crys}, 1}+n_{\mathrm{crys}, 5}-n_{\mathrm{crys}, 3}-n_{\mathrm{crys}, 7} . \\
& v=n_{\mathrm{crys}, 2}+n_{\mathrm{crys}, 6}-n_{\mathrm{crys}, 4}-n_{\mathrm{crys}, 8} .
\end{aligned}
$$




$$
w=n_{\text {crys }, 5}+n_{\text {crys }, 6}+n_{\text {crys }, 7}+n_{\text {crys }, 8}-n_{\text {crys }, 1}-n_{\text {crys }, 2}-n_{\text {crys }, 3}-n_{\text {crys }, 4} .
$$

Using Equation 3.7 and 3.8 below, this direction vector is converted into spherical polar coordinates:

$$
\text { Azimuth }=\arctan \left(\frac{v}{u}\right)-45^{\circ}
$$

and,

$$
\text { Elevation }=\arccos \left(\frac{w}{\sqrt{u^{2}+v^{2}+w^{2}}}\right)-90^{\circ} .
$$

The elevation angle spans from $-90^{\circ}$ (directly below ARDUO) to $90^{\circ}$ (directly above ARDUO), and the azimuth angle spans $360^{\circ}$ clockwise along the horizon from the nose direction of the UAS. These angles with respect to the ARDUO orientation are shown in Figure 2.5b.

\section{Heading Correction}

As the Responder and UAS is flying, the nose of the Responder, which is in-line with the ARDUO's $0^{\circ}$ azimuth angle axis, can be rotated from the flight direction due to windy conditions or during turns. This rotation is logged by the autopilot as the yaw, the orientation angle of the nose of the Responder with respect to geographical north. The direction vector's azimuth angle is calculated with respect to the yaw angle of the Responder at the time the data point is collected. As the yaw changes throughout the survey, each azimuth angle needs to be converted to an angle with respect to geographical north to establish a common reference among all data points. The direction vector's azimuth angle with respect to the ARDUO is added to the yaw at the corresponding time to convert it to an azimuth angle with respect to geographical north. 


\section{Direction Vector Overlay}

The direction vectors are visualized in two dimensions and overlaid on the count-rate map. Each direction vector is displayed as an arrow with the arrowhead at the data point location. The arrow is rotated to point in the direction of the azimuth angle with respect to geographical north. The length of the arrow represents the elevation angle, with shorter arrows being closer to vertical, and longer arrows being closer to horizontal. The arrow colour indicates whether the elevation angle is positive (upwards) or negative (downwards).

\subsubsection{Direction Vector Projection}

An additional processing step using the direction vectors can be done to improve on source localization. Each direction vector is projected to the ground surface, and a new point is created where the vector intersects the ground. Recall that the elevation angle reconstruction is limited as it approaches the horizon. This was shown in the deviation in the reconstructed elevation angle from the true elevation angle in Figure $2.16 \mathrm{~b}$. For this reason, a cut on elevation angles above $-40^{\circ}$ is used to remove the effect from poorly-reconstructed elevation angles. Moreover, the restriction to downward elevation angles approaching vertical minimizes the affect of altitude fluctuations or offsets on the calculation of the location of the intersection point with the ground.

A diagram illustrating this process is shown in Figure 3.5. The locations of each data point at an altitude above ground level are shown by the helicopters. The direction vector is shown by the orange arrow extending from the ARDUO to the ground, shown by the blue surface. While a flat surface is shown in Figure 3.5, the method is extendable to terrain with topographic variations by using a digital elevation model. The direction vector's intersection point with the ground is calculated using the Responder UAS's orientation and position from the autopilot, and the direction vector's 
azimuth and elevation angles. The kernel density function provided by ArcGIS [9] is used to contour the spatial point density of the intersection points, where each point is weighted by the number of gamma-ray energy deposits at the measurement location. Areas with higher point densities indicate possible source locations.

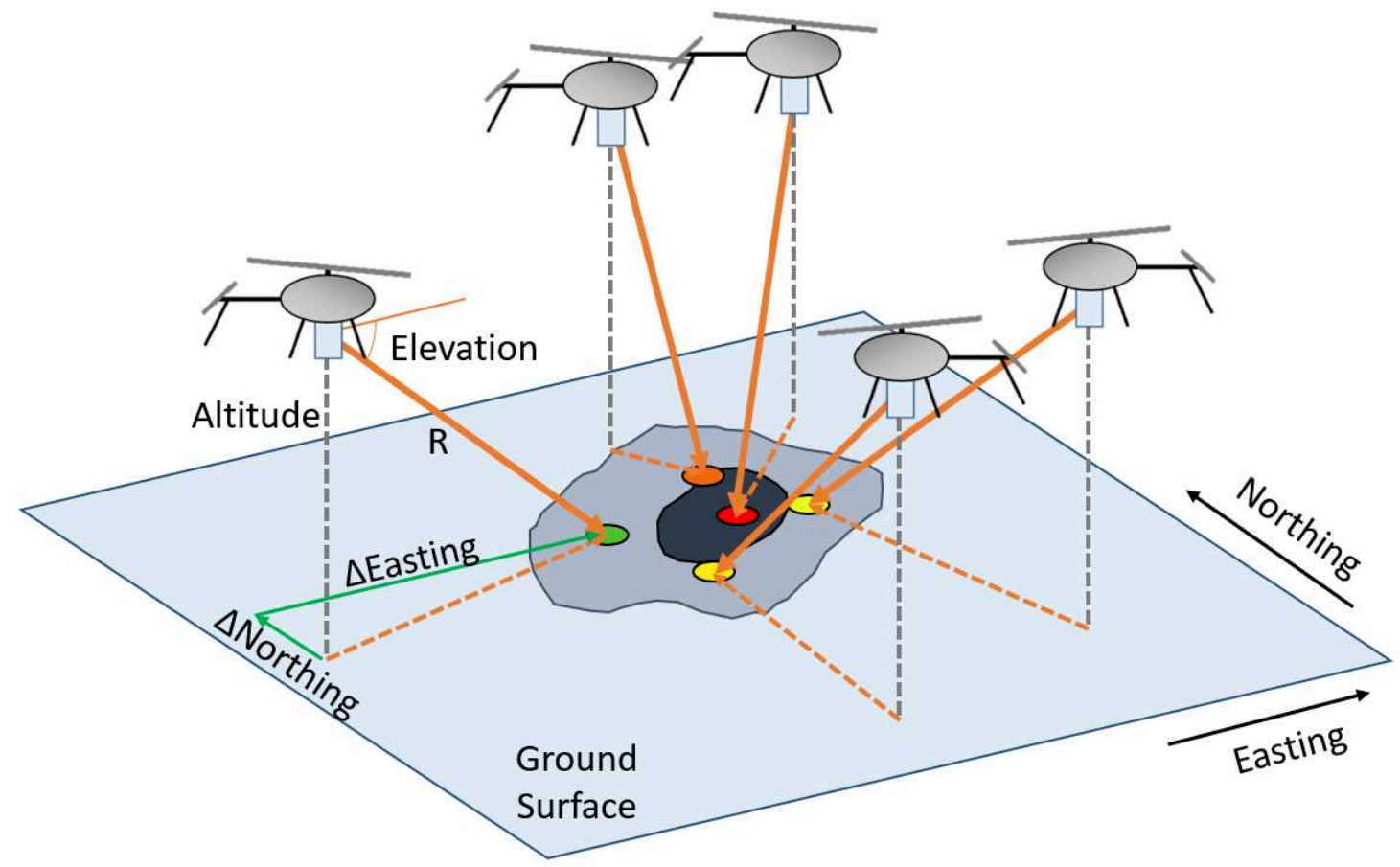

Figure 3.5: Visualization of the process of projecting the direction vectors collected during flight down to a ground surface. The ground surface is represented by the blue plane. The direction vector is represented by the orange arrow that is projected from the ARDUO under the helicopter to the ground surface. The coloured points are the locations where the direction vectors intersect the ground. They are coloured based on the original data point's count rate, with red indicating a higher count rate and green indicating a lower count rate. The darker blue contours on the ground surface represent the spatial point density contour results from the ArcGIS [9] kernel density analysis. The contour with the darkest blue area indicates the most likely source location.

\subsubsection{Source Strength Estimation}

A source strength estimation was conducted for the point source trial as well as for the distributed source trial. As the source geometry was different for each trial, a separate 
processing method is needed for each geometry to estimate the source strength. The two processing methods - one for a point source geometry, and one for a distributed source geometry - are presented in the following subsections.

\section{Point Source Strength Estimation}

Furthering the direction vector projection method, the vectors can be used to estimate the source strength at the predicted source location. The source strength analysis is only completed for the single ${ }^{137}$ Cs point source trial presented in Section 4.2.1 as a proof that this method is possible. The determination of the source strength of multiple point sources or a distributed source is complex and outside the scope of this thesis. Additionally, only the point closest to the true source location was analyzed as the intrinsic photopeak efficiency of the ARDUO has only been determined for a source located directly below, at an elevation angle of $-90^{\circ}$. For data point locations at other elevation angles, an intrinsic photopeak efficiency value for that elevation angle would need to be known before a source strength could be calculated. A new intrinsic photopeak efficiency would also need to be calculated if the source was not ${ }^{137} \mathrm{Cs}$.

To calculate the source strength, the first step is to calculate the distance between the data point and the direction vector intersection with the ground. The inverse square law, attenuation in air, and the spectrometer's intrinsic photopeak efficiency are then used. The source strength, $A(\mathrm{~Bq})$, is then estimated using,

$$
A=\frac{n_{\mathrm{p}, i}}{\mathrm{BR}} \cdot \frac{1}{\epsilon_{\mathrm{int}}(E)} \cdot \frac{4 \pi R^{2}}{A_{\mathrm{ARDUO}}} \cdot \frac{1}{e^{-\mu_{\mathrm{air}}(E) R}}
$$

where $n_{\mathrm{p}, i}$ (cps) is the photopeak count rate of data point $i$ at its original location, $\mathrm{BR}$ is the branching ratio for the gamma ray energy of the isotope, $R$ is the distance between the data point and its direction vector ground intersection point $(\mathrm{m}), \epsilon_{\mathrm{int}}(E)$ is the intrinsic efficiency of the ARDUO at the energy of interest, $A_{\mathrm{ARDUO}}$ is the 
estimated effective surface area of the $\operatorname{ARDUO}\left(\mathrm{m}^{2}\right)$, and $\mu_{\text {air }}(E)$ is the attenuation coefficient for air at the energy of interest $\left(\mathrm{m}^{-1}\right)$. For this case the estimated effective surface area of the ARDUO is equal to the area of the small face of four of the crystals. The attenuation coefficient for air is determined from the data provided by the National Institute of Standards and Technology [36].

\section{Surface Activity Concentration Mapping}

The surface activity concentration analysis can be conducted if the source is distributed, therefore this analysis is only completed for the distributed source trial presented in Chapter 5. If the source geometry is not known, the photopeak count rate map can be examined to try and determine the source geometry.

The surface activity concentration, $A_{\mathrm{S}, i}\left(\mathrm{MBq} \cdot \mathrm{m}^{-2}\right)$, for each data point $i$ is calculated using the photopeak count rate, $n_{\mathrm{p}, i}(\mathrm{cps})$, and the sheet source sensitivity of the ARDUO, $S_{i}\left(\mathrm{cps} /\left[\mathrm{MBq} \cdot \mathrm{m}^{-2}\right]\right)$, where

$$
A_{\mathrm{S}, i}=\frac{n_{\mathrm{p}, i}}{S_{i}} .
$$

The sheet source sensitivity at an energy of $1.596 \mathrm{MeV}$ (the energy of the ${ }^{140} \mathrm{La}$ photopeak used in the distributed source analysis) is calculated as a function of altitude using Equation 2.5 in Section 2.2.6. Each data point is mapped based on its location in UTM, as described in the previous section. The surface activity concentration for each data point is interpolated using the inverse distance weighted (IDW) method [41]. If a different isotope or a different ${ }^{140} \mathrm{La}$ photopeak were used, a new sensitivity function would need to be calculated.

The surface activity concentration map can be used to estimate the total activity in the area. The surface activity concentration calculation is based on the assumption that the source is an infinite sheet of radiation. When the measured count rate is converted to a surface activity concentration for a source of limited extent, it results in 
an under-estimation of the surface activity concentration in the area above the source, and an overestimation of the surface activity concentration in the area where no source is present. While the calculated surface activity concentration not representative of the true surface activity concentration of a source of limited extent at individual locations, the total integrated activity of the source can be calculated and correctly represented. This is done by numerical integration the surface activity concentration at a $1 \mathrm{~m}^{2}$ grid spacing over the total area of the surface activity concentration that is attributed to the source. The integrated result is the total activity of the source. 


\section{Chapter 4}

\section{Point Source Trials}

Following the laboratory characterization of the ARDUO, a series of in-flight tests were conducted at the NRCan Geomagnetic Laboratory in Ottawa, Ontario. Flights were conducted over a single point source of ${ }^{137} \mathrm{Cs}$, and over two point sources of ${ }^{137} \mathrm{Cs}$. The results from these experiments are discussed in this chapter.

\section{$4.1 \quad$ Experimental Setup}

A survey area of $110 \mathrm{~m} \times 50 \mathrm{~m}$ was used for the small scale point source tests. The area is located in the northern section of the NRCan Geomagnetic Laboratory compound in Ottawa. The compound is located on surficial deposits of high-terrace alluvium composed of non-calcareous, non-fossiliferous medium to fine sands [42]. The sands are associated with abandoned river channels, which are now partially occupied by the Mer Bleue Peat Bog in the surrounding area. A background geological signal from the ${ }^{40} \mathrm{~K}$ in the sand minerals is expected for the area.

The survey area was relatively flat, and clear of trees and bushes. A laboratory building in the compound near the survey area was used for powering the GCS and charging the UAS batteries. In remote locations, a portable generator would be used for a power source. The surveys were conducted in September 2018. Weather conditions were dry, with partial cloud cover. There was a small breeze of $2 \mathrm{kt}$, 
with some gusts up to $5.8 \mathrm{kt}$. These wind conditions are well within the operational conditions for flying the Responder UAS. Three flights were conducted: a survey over a single ${ }^{137} \mathrm{Cs}$ point source, a survey over two ${ }^{137} \mathrm{Cs}$ point sources, and a background survey without the sources present.

The point sources were placed at specific locations within the survey area. The locations, listed in Table 4.1, were surveyed to an accuracy of $0.011 \mathrm{~m}$ in Easting, and $0.017 \mathrm{~m}$ in Northing (Issam Al-Khoubbi, written communication, September 5, 2018).

Table 4.2 summarizes the surveys that were completed. The first survey was conducted over two point sources of ${ }^{137} \mathrm{Cs}$, with activities at the time of surveying of approximately $161 \mathrm{MBq}$ each, placed at locations PYL1 and PYL2. The sources were separated by a distance of approximately $27 \mathrm{~m}$. The second survey conducted was the background survey, where the sources were removed from the survey area and stored in their shielded containers. The third flight was over a single point source of ${ }^{137} \mathrm{Cs}$ with an activity of approximately $161 \mathrm{MBq}$ at the time of the survey at location PYL1. When surveying, the point sources were placed on top of $0.3 \mathrm{~m}$ tall traffic cones for visibility. The traffic cone height was subtracted from the measured altitude from the Responder autopilot for more accurate source strength estimations.

Table 4.1: Point source locations in UTM Zone 18.

\begin{tabular}{ccc}
\hline Location ID & Easting $(\mathrm{m})$ & Northing $(\mathrm{m})$ \\
\hline PYL1 & $456789.145 \pm 0.011$ & $5028344.253 \pm 0.017$ \\
PYL2 & $456814.597 \pm 0.011$ & $5028335.335 \pm 0.017$ \\
\hline
\end{tabular}

The UAS was flown using the same flight plan for each of the three surveys. The flight plan is shown in Figure 4.1 overlaid on a map of the survey location. The flights were conducted at a nominal altitude of $10 \mathrm{~m}$ above ground level, with a nominal 
speed of $2 \mathrm{~m} / \mathrm{s}$ and a line spacing of $4 \mathrm{~m}$. Each flight took approximately $14 \mathrm{~min}$ from takeoff to landing.

The UAS took off from the paved path, located to the south of the survey grid, as it provided a flat surface free of obstacles. Once the UAS reached a nominal altitude of $30 \mathrm{~m}$, the set takeoff altitude for this survey, the UAS flew towards center of the survey grid, and then proceeded towards the north west corner of the grid pattern while descending to a nominal altitude of $10 \mathrm{~m}$. The UAS then flew the grid pattern at a nominal altitude of $10 \mathrm{~m}$. Upon completion of the survey grid at the southwestern corner of the grid, the UAS flew to the middle of the grid while ascending to a nominal altitude of $30 \mathrm{~m}$. The UAS then flew to a location near the takeoff location, and executed its 'Return to Launch' command, where it flew back to the takeoff location and landed.

The data collected while the UAS was flying to and from the grid pattern was excluded from the data processing procedures, therefore only the data from the grid pattern was used.

Table 4.2: Summary of the flights conducted for the point source trials.

\begin{tabular}{cccc}
\hline Date & Start Time (EDT) & Flight Duration (min:sec) & Source Configuration \\
\hline \hline $06 / 09 / 2018$ & $12: 52$ & $14: 24$ & PYL1 and PYL2 \\
\hline $06 / 09 / 2018$ & $13: 52$ & $14: 24$ & No source \\
\hline $06 / 09 / 2018$ & $15: 26$ & $14: 25$ & PYL1 \\
\hline \hline
\end{tabular}

Weather Conditions: Partial cloud, $25^{\circ} \mathrm{C}, 101.43 \mathrm{kPa}, 2 \mathrm{kt}$ to $6 \mathrm{kt}$

\subsection{Results}

The flight data for the surveys were processed according to the procedures described in Section 3.2. 


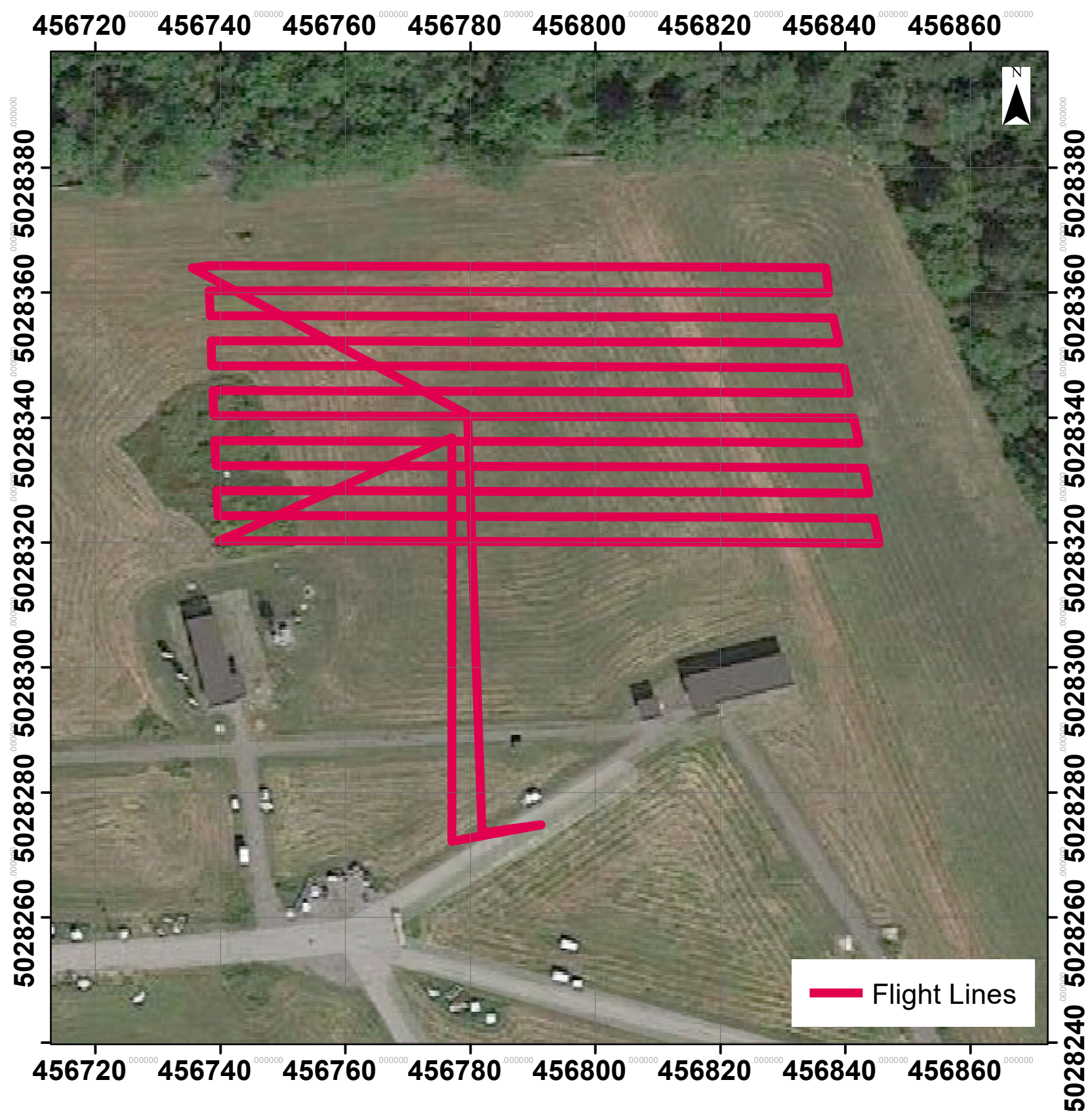

Figure 4.1: Survey area in the compound of the NRCan Geomagnetic Laboratory. The planned flight lines are shown by the red lines. The UAS takes off from the paved path connecting the buildings. The survey grid is over a relatively flat area. The survey grid has a line spacing of $4 \mathrm{~m}$ with line lengths of approximately $110 \mathrm{~m}$. The map grid spacing is $20 \mathrm{~m}$. Imagery from Google Earth [43].

The background survey over the area is shown in Figure 4.2. The photopeak count rate is shown by the interpolated map. The map shows that the background photopeak count rate is relatively constant within the survey area, as it has a maximum 
range of $7 \mathrm{cps}$. The maximum photopeak count rate in the area is $10 \mathrm{cps}$, which is significantly lower than the photopeak count rate when a source is present. The direction vectors that are calculated using a total count rate are overlaid on the map. The vectors are oriented randomly throughout the survey area, indicating that there is no significant trend or anomaly in the background count rate.

To obtain a survey result that is only representative of the signal from the point source, the average background count rates in the total-count and isotope-dependent energy windows were subtracted from each point source survey data point's count rate in the same energy windows. Subtracting the average background count rate, rather than subtracting an interpolated background count rate grid value at each point source survey data point location, was considered adequate due to the low variability in the background count rate throughout the survey area. The use of the average background count rate also reduced the data processing complexity.

Figure 4.3 shows representative $1 \mathrm{~s}$ spectra from the signal, average background and background subtracted signal. The spectra are shown for each of the eight crystals of the ARDUO, as well as for the sum of the eight crystals. The spectra data were collected with a low energy cutoff of approximately $0.05 \mathrm{MeV}$, therefore no spectral information is displayed near zero. The signal spectra are for the survey over one ${ }^{137} \mathrm{Cs}$ source. Similar spectra are produced for the survey over two ${ }^{137} \mathrm{Cs}$ sources. The $0.662 \mathrm{MeV}$ photopeak from the source is shown in the signal spectrum. The energy window for the photopeak count-rate analysis is set from $0.610 \mathrm{MeV}$ to $0.713 \mathrm{MeV}$, approximately three standard deviations centered around the photopeak energy. The red dotted lines overlaid on the spectra represent the photopeak energy window bounds. The energy window is properly confining the photopeak. There is a significant amount of low energy Compton scattering that is produced by gamma rays from the source which are incompletely measured. The average background count rate is also shown in Fig 4.3, by the solid green spectrum. The count rates may be significantly lower in 


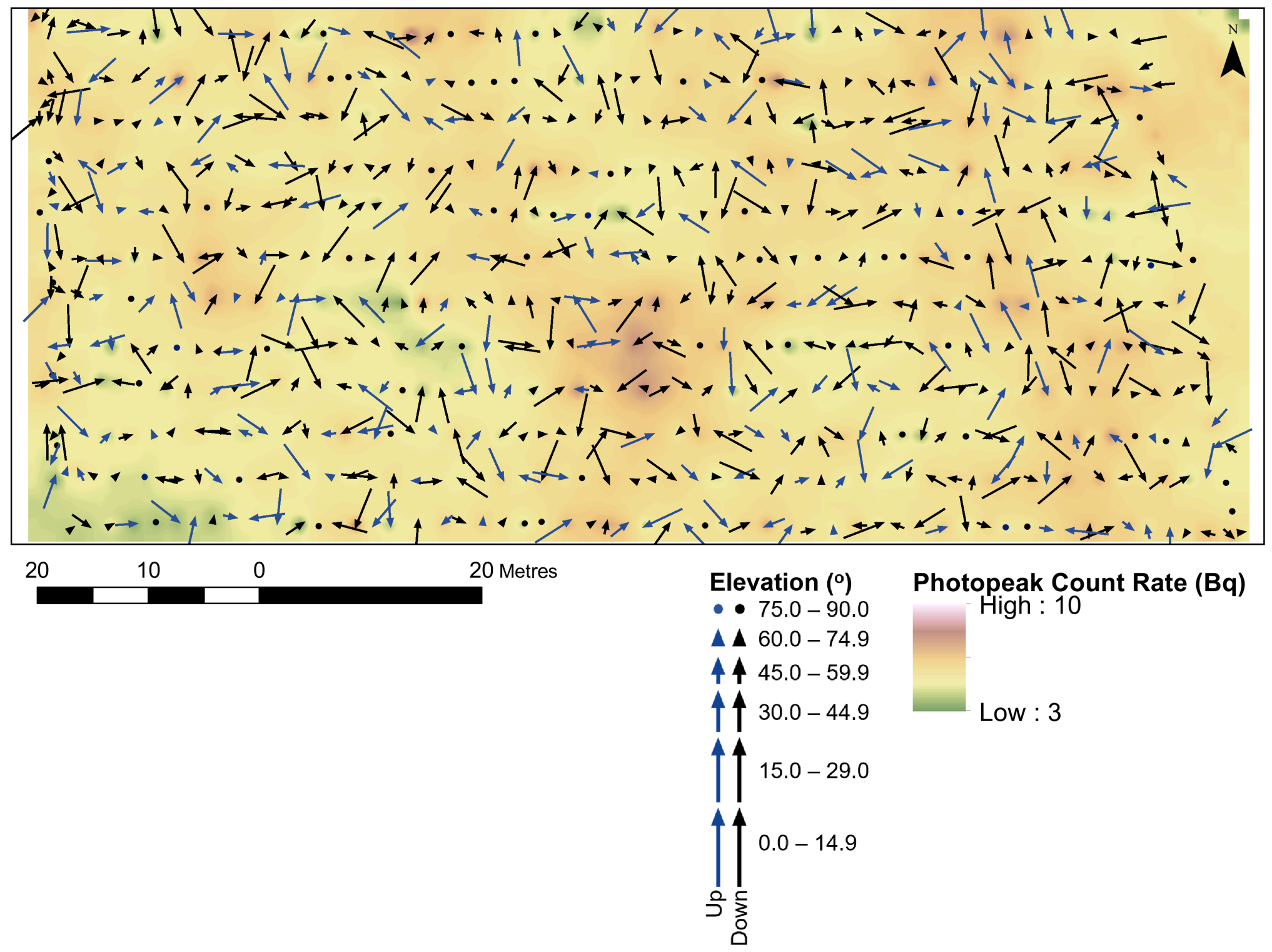

Figure 4.2: Background survey for the point source trials. The photopeak count rates are shown by the interpolated map with red and white representing a higher count rate, and green representing a low count rate. The direction vectors calculated using a total count rate are overlaid on the map. 
some crystals of the ARDUO when compared to the other crystals. This information allows the reconstruction of the direction vector to the source.

The signal, background, and background subtracted spectra averaged over the entire survey for crystal 1 of the ARDUO for the one ${ }^{137} \mathrm{Cs}$ survey are shown in Figure 4.4. Similar spectra are produced in the other 7 crystals of the ARDUO and for the two ${ }^{137} \mathrm{Cs}$ survey. The photopeak from the ${ }^{137} \mathrm{Cs}$ source is shown in the point source signal data, and the 3 standard deviation energy window bounds are represented by the dotted lines. The ${ }^{137} \mathrm{Cs}$ source does not appear in the background signal. The difference between the total signal count rate and the average background count rate represents the signal due to the ${ }^{137} \mathrm{Cs}$ source.

A ${ }^{40} \mathrm{~K}$ photopeak is expected in the background due to the potassium present in the sand minerals of the area. Figure 4.4 shows that the $1.460 \mathrm{MeV}$ photopeak from ${ }^{40} \mathrm{~K}$ is present in both the signal and background spectra, which were normalized to their respective total accumulation times. The ability of the background spectrum to fully account for the count rate of the ${ }^{40} \mathrm{~K}$ photopeak and for all the count rates above the ${ }^{137} \mathrm{Cs}$ photopeak demonstrates that the spectrum is representative of the background under the total signal curve. This also indicates that the background survey can be used to subtract the geological effects from the point source survey. In addition to the low variability of the background count rate map shown inFigure 4.2, the average background spectrum further demonstrates that the average background value well defines the background in the whole survey area.

\subsubsection{One ${ }^{137}$ Cs Source Survey}

The background-subtracted photopeak count rate map is shown in Figure 4.5. The photopeak count rate is interpolated, and displayed in the colour scale where green is low, and white is high. The direction vectors for each data point are calculated using 

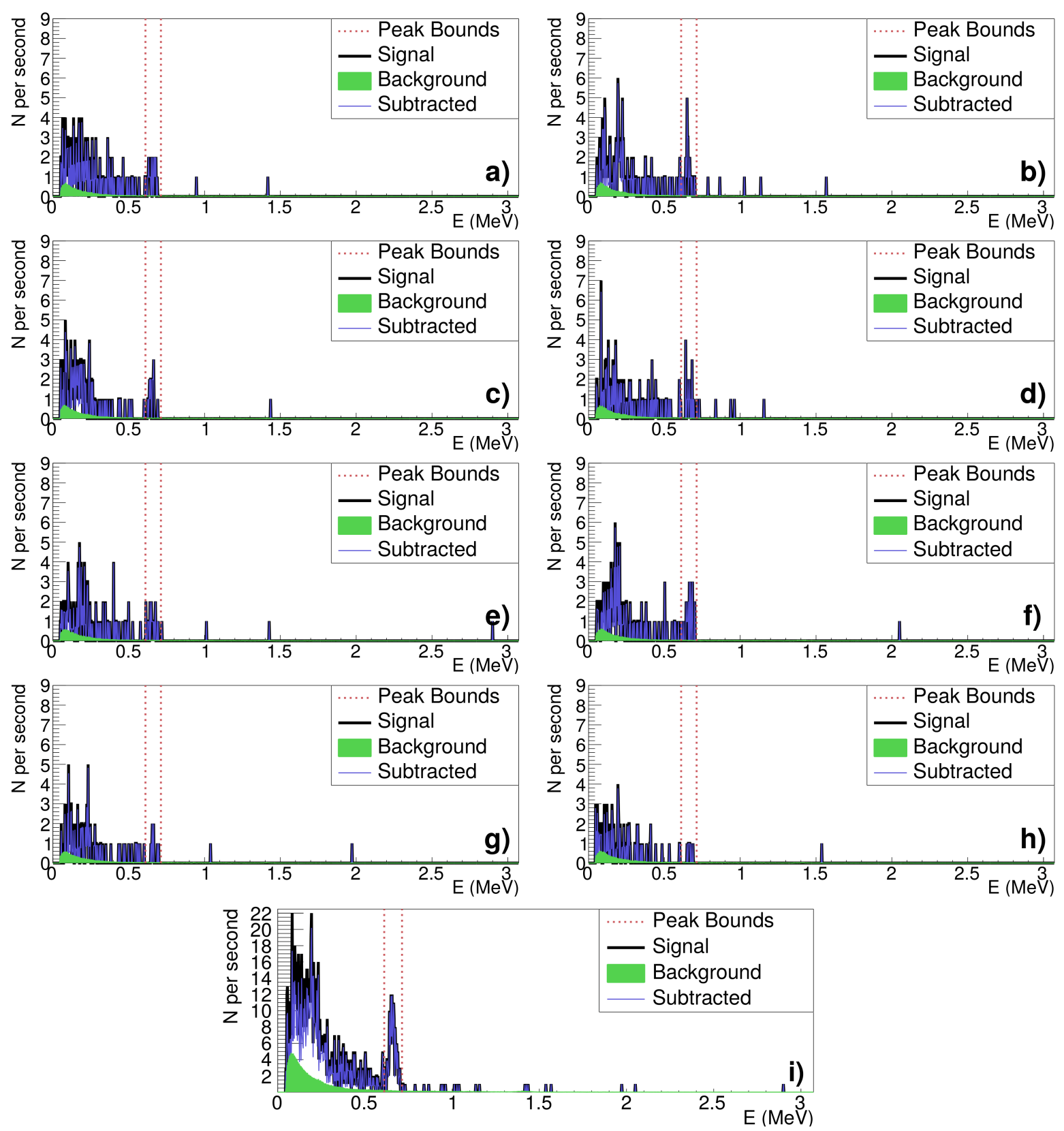

Figure 4.3: Representative $1 \mathrm{~s}$ energy spectra for a data point located over the ${ }^{137} \mathrm{Cs}$ source for the survey over one point source of ${ }^{137} \mathrm{Cs}$. The $\mathrm{y}$-axis is the number of counts, $\mathrm{N}$, per second. The $\mathrm{x}$-axis is the energy, $\mathrm{E}$, in $\mathrm{MeV}$. The $1 \mathrm{~s}$ signal spectrum is shown by the black line. The average background energy spectrum for the survey area is shown by the green-filled area. The signal with the average background subtracted is shown by the blue line. The energy window bounds used for the photopeak analysis are shown by the red dotted lines. a) to h) Representative $1 \mathrm{~s}$ energy spectra from each of the eight ARDUO crystals, 1 to 8 respectively. i) Total $1 \mathrm{~s}$ spectra from the sum of the eight crystals. 


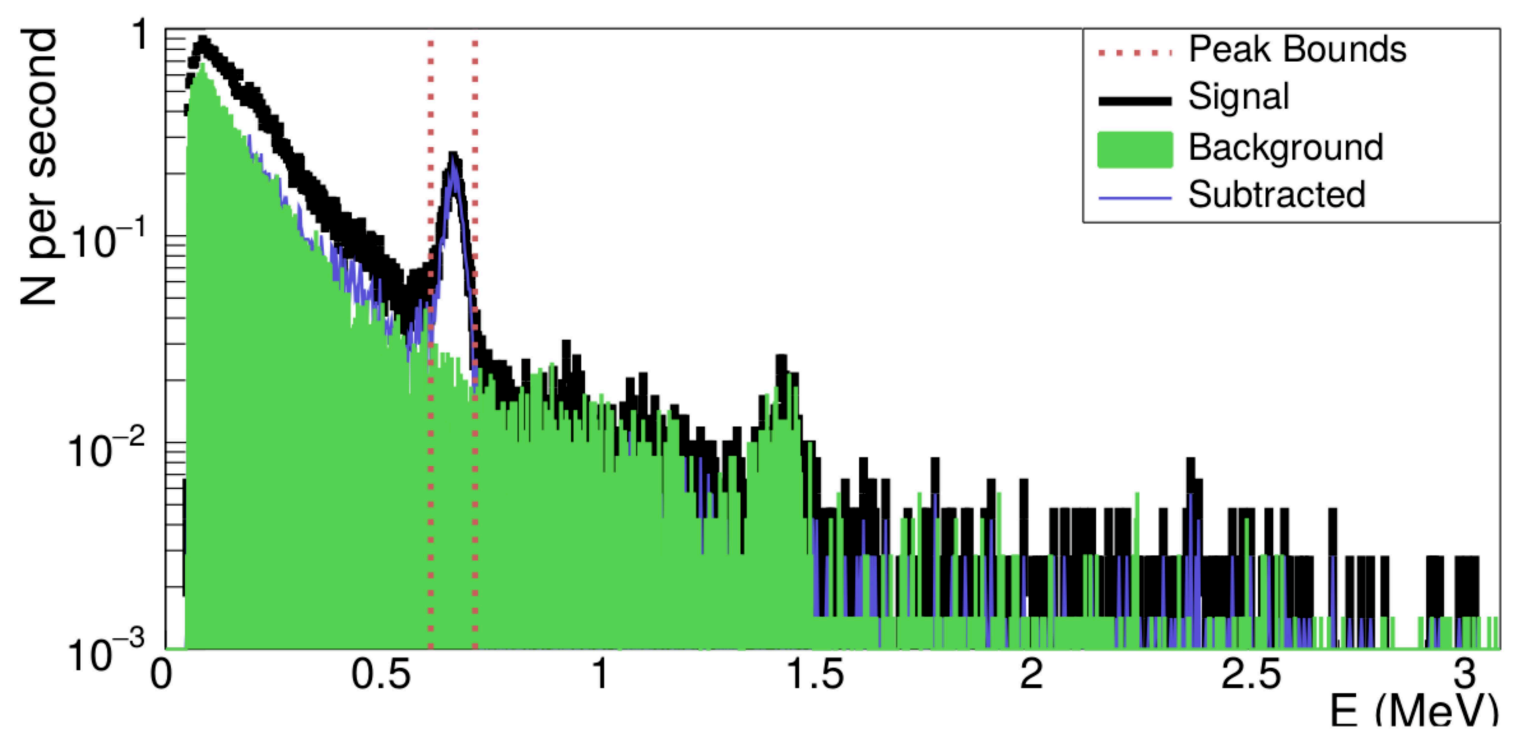

Figure 4.4: Representative average survey energy spectra from crystal 1 of the ARDUO for the single ${ }^{137} \mathrm{Cs}$ point source survey. The $\mathrm{y}$-axis is the number of counts, $\mathrm{N}$, per second in a log-scale. The $\mathrm{x}$-axis is the energy, $\mathrm{E}$, in $\mathrm{MeV}$. The average background signal is shown by the filled green spectrum. The $1.460 \mathrm{MeV}$ photopeak from ${ }^{40} \mathrm{~K}$ is shown in both the signal and background spectra.

the total count energy window and overlaid on the count rate map. The arrowhead of each direction vector is placed at the lag-corrected data point location. Upwards pointing arrows are shown in blue, with downwards arrows shown in black. The true source location is indicated by the purple diamond.

The photopeak count rate indicates that there is a high radially symmetric anomaly located in the south-east corner of the map. The location of the maximum of this anomaly corresponds well with the true source location. The anomaly has a maximum spatial extent of approximately $30 \mathrm{~m}$, after which the count rate is at background level.

When examining the direction vectors overlaid in Figure 4.5, it can be seen that they are oriented randomly when the count rate is low. As the UAS approaches the source, the direction vectors orient themselves towards the source in both the azimuth and elevation angles. The direction vectors show elevation angles near the horizon as the UAS enters the field of influence of the source. As the count rate increases, 
the elevation angle decreases, approaching a near vertical downwards angle at the maximum of the anomaly. For the data points located closest to the true source location, the direction vector is pointing straight downwards. The direction vector information is consistent with the photopeak count rate interpolation, as well as with the true source location.

The projection map is shown in Figure 4.6. The direction vectors are projected to the ground surface, and the intersection points are indicated by the coloured circles. The colour scale represents the total count rate, with red indicating a high rate and green indicating a low rate. The map shows a high concentration of red circles in the south-east corner of the survey area. The rest of the survey area contains low count rate intersection points, with an even distribution around the area. The blue contour on the map is the result from the point density analysis. The darker blue indicates a higher point density, with a high density anomaly present around the concentration of high count rate intersection points. Both the intersection points and the point density anomaly are consistent with the true source location, shown by the purple diamond. The projection method is effective in reducing the spatial extent of the measured anomaly from approximately $30 \mathrm{~m}$ to less than $10 \mathrm{~m}$.

A simplified source strength estimation was completed for the single point source survey as a proof of concept of the method. This analysis was only completed for the single source survey as the other point source survey has the added complexity of multiple point sources. The data point closest to the true source location and with a reconstructed elevation angle closest to $-90^{\circ}$ was used for the analysis. The corresponding intrinsic photopeak efficiency of the ARDUO has been determined for a location directly below the spectrometer as reported in Chapter 2, Section 2.2.5. The use of other data point locations would require a detailed analysis of the intrinsic 

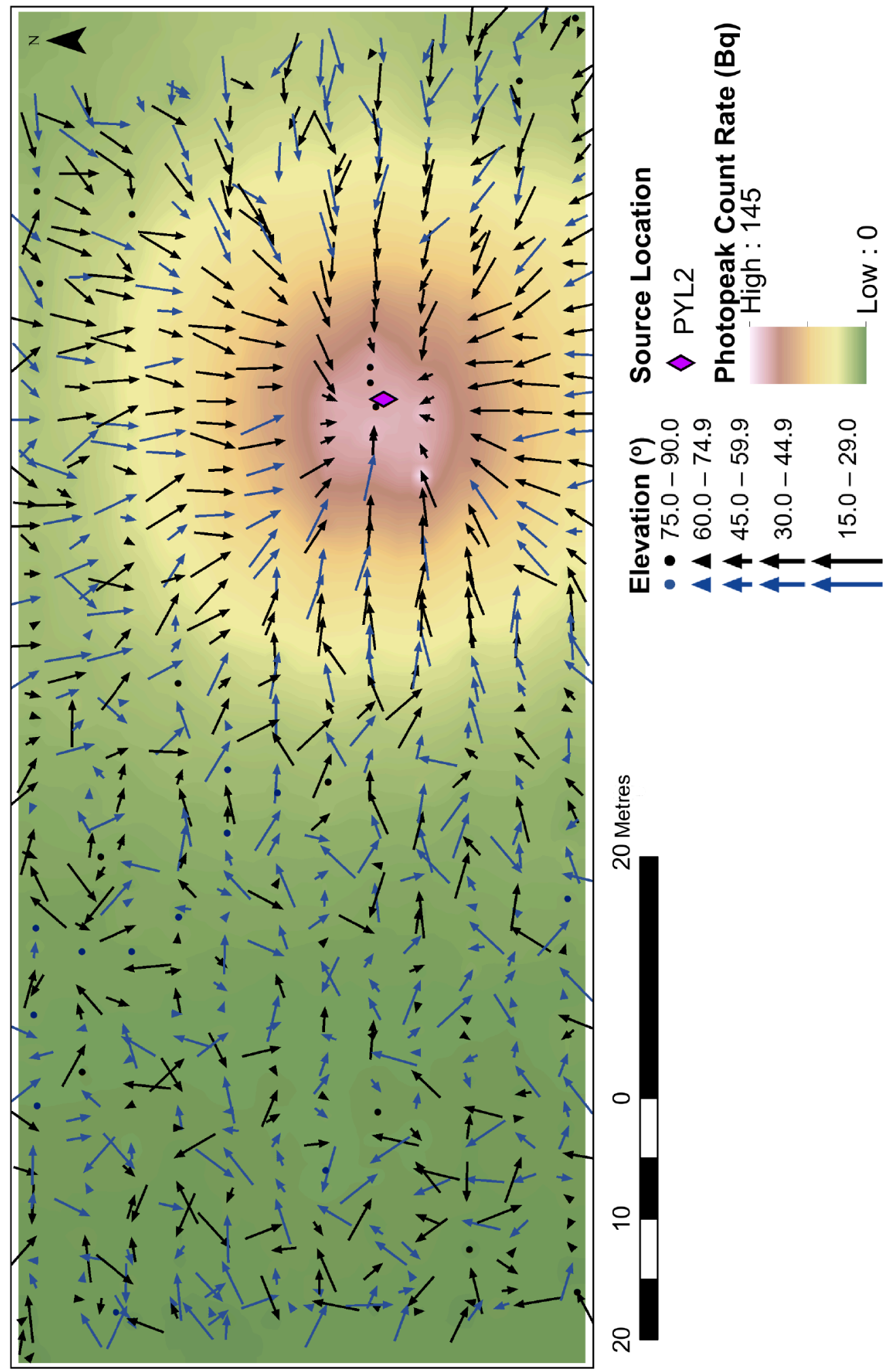

己

$\Xi 8$

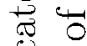

.气.

.

0

苛

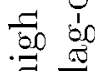

$<\frac{9}{1}$

$\dot{D} \cdot \frac{10}{2}$

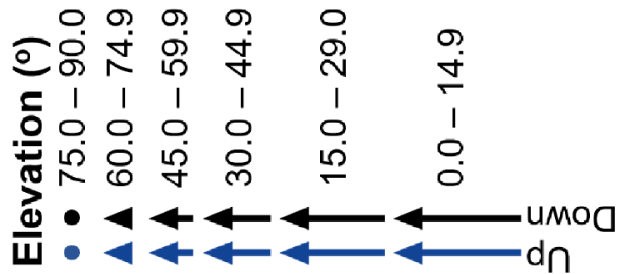

胥

$\div$

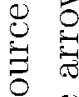

o 0

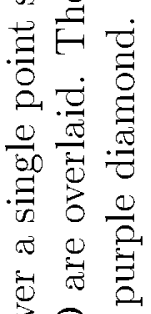

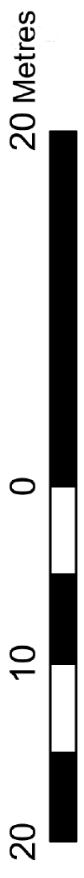

웜요

s.

$\exists$ 更

$\sigma \stackrel{0}{0}$

छ

莤的

छ ธิ

$\stackrel{8}{0}$

¿응

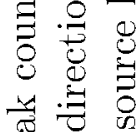

吕导

过

$\because \frac{3}{2}$.

ن

$\underset{0}{0}$ 


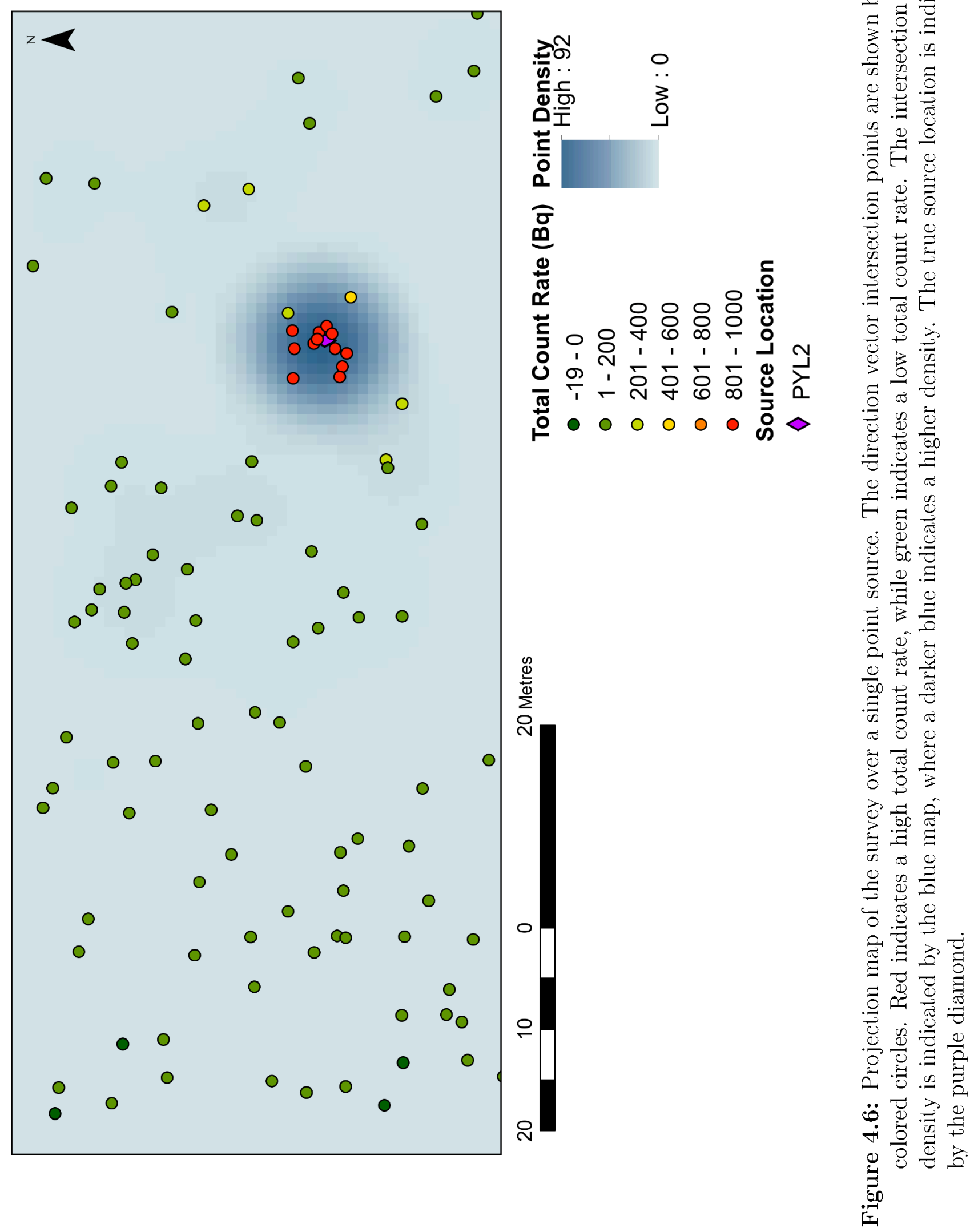


photopeak efficiency of the ARDUO in all directions. The values used to calculate the source strength using Equation 3.9 in Section 3.2.10 are shown in Table 4.3.

The altitude has the largest impact on the source strength, therefore the uncertainty on the extracted source strength was determined through an analysis of the altitude using the process described in Section 3.2.6. Figure 4.7 shows the distribution of the altitude measurements for the single point source survey. The $0.3 \mathrm{~m}$ altitude offset from the autopilot's altitude to the altitude of the ARDUO has been subtracted from all altitude measurements. The $0.3 \mathrm{~m}$ offset from the source being placed on a traffic cone has also been subtracted from the altitude measurements. The range of accepted altitudes corresponding to a maximum $10 \%$ change in the count rate due to a change altitude is shown by the solid vertical green lines. The accepted altitude range is from $8.93 \mathrm{~m}$ to $9.87 \mathrm{~m}$. The dashed blue line shows the mean of the distribution at an altitude of $9.40 \mathrm{~m}$. The acceptable altitude range contains the majority of the data points. This indicates that the stochastic fluctuations in the reported altitude are within the accepted limit.

In addition to the stochastic fluctuations, there may also be a systematic bias in the altitude measurement. This may be caused by a lower precision and higher uncertainty in the barometric pressure measurement as well as in the GNSS vertical component measurement used to calculate the altitude. From visual observation of flights with the UAS, it is estimated that there may be a systematic bias of approximately $1 \mathrm{~m}$. From this, the uncertainty in the altitude due to a potential systematic bias is therefore estimated to be approximately $1 \mathrm{~m}$. This $1 \mathrm{~m}$ uncertainty on the altitude was used to estimate the uncertainty on the source strength estimation.

The final source strength estimation for a point nearly directly above the source of $175 \mathrm{MBq} \pm 39 \mathrm{MBq}$. This is within range of the true source strength of $161 \mathrm{MBq}$, indicating that this method can be used for an estimation of the source strength. 


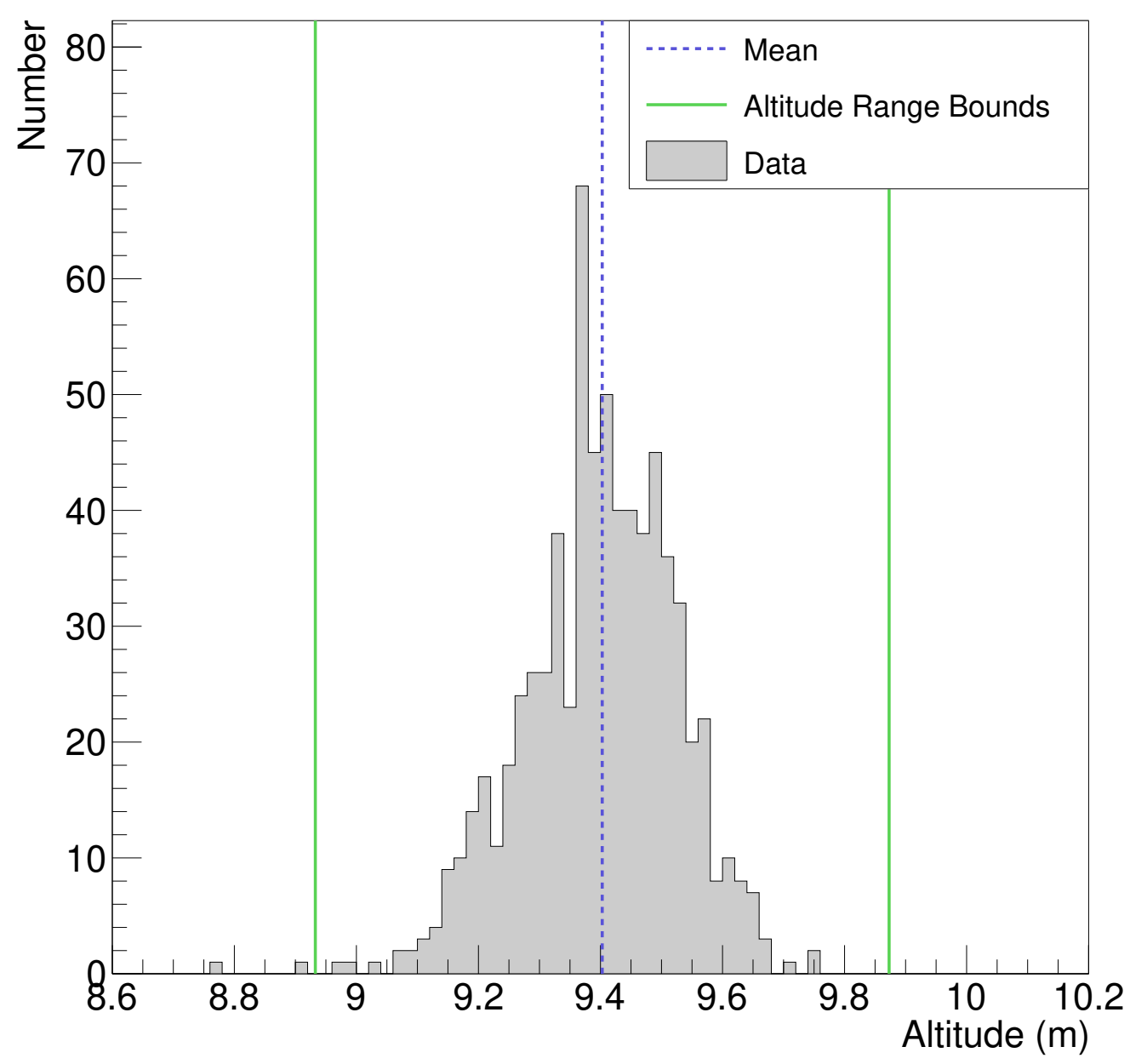

Figure 4.7: Histogram of the altitude measurements for the single ${ }^{137} \mathrm{Cs}$ point source survey. The mean of the altitude distribution is shown by the blue dashed line. The altitude range boundaries that correspond to a $10 \%$ change in the count rate due to a change in the altitude are shown by the solid green lines.

Table 4.3: Source strength estimation values.

\begin{tabular}{cccccc}
\hline Data Point ID & $n_{p}(\mathrm{cps})$ & Altitude $(\mathrm{m})$ & Elevation $\left(^{\circ}\right)$ & $R(\mathrm{~m})$ & $\begin{array}{c}\text { Source Strength } \\
(\mathrm{MBq})\end{array}$ \\
\hline 427 & 122 & 9.45 & -87 & 9.46 & $175 \pm 39$ \\
\hline
\end{tabular}

\subsubsection{Two ${ }^{137}$ Cs Sources Survey}

Figure 4.8 shows the count rate map with the overlaid direction vectors and source locations for the two ${ }^{137} \mathrm{Cs}$ source survey. A high photopeak count rate in the ${ }^{137} \mathrm{Cs}$ 
peak is shown in white and red, and a low photopeak count rate is shown in green. The true source locations are indicated by the pink and purple diamonds. The reconstructed direction vectors are overlaid on the count rate distribution in Figure 4.8. The arrowhead of the direction vector is at the lag-corrected location of each data point. The length of the vector represents its elevation angle, with the shorter arrows indicating a more vertical elevation angle. The blue arrows have positive elevation angles, and are upwards pointing. The black arrows have negative elevation angles, and are downwards pointing.

The spatial distribution of the count rate shows an elongated anomaly extending diagonally north-west to south-east across the survey area. The highest count rates are located at either end of the anomaly. The high count rate anomaly corresponds well with the true locations of the point sources, however, without the true source location information, the count rate map could be interpreted to be the result of a distributed source.

The overlaid direction vectors provide additional information about the spatial distribution of the anomaly. The vectors tend to point towards two separate locations on the map, corresponding to the two ends of the elongated anomaly. The direction vectors located between the two true source locations do not point downwards as would be expected from a distributed source. The vectors instead point to locations away from the middle of the count rate anomaly and toward the two actual source locations near the ends of the anomaly. Thus, by examining the direction vectors, it can be surmised that the count rate distribution is being caused by two separate sources.

Figure 4.9 shows the results from the direction vector projection and point density analysis. The pink and purple diamonds indicate the true source location. Using the 






Figure 4.8: Photopeak count rate map from a survey over two point sources of ${ }^{137} \mathrm{Cs}$. A high count rate is indicated in red and white. The direction vectors from the ARDUO are overlaid. The arrowhead is the lag-corrected location of the data point. The true source locations are indicated by the pink and purple diamonds. 

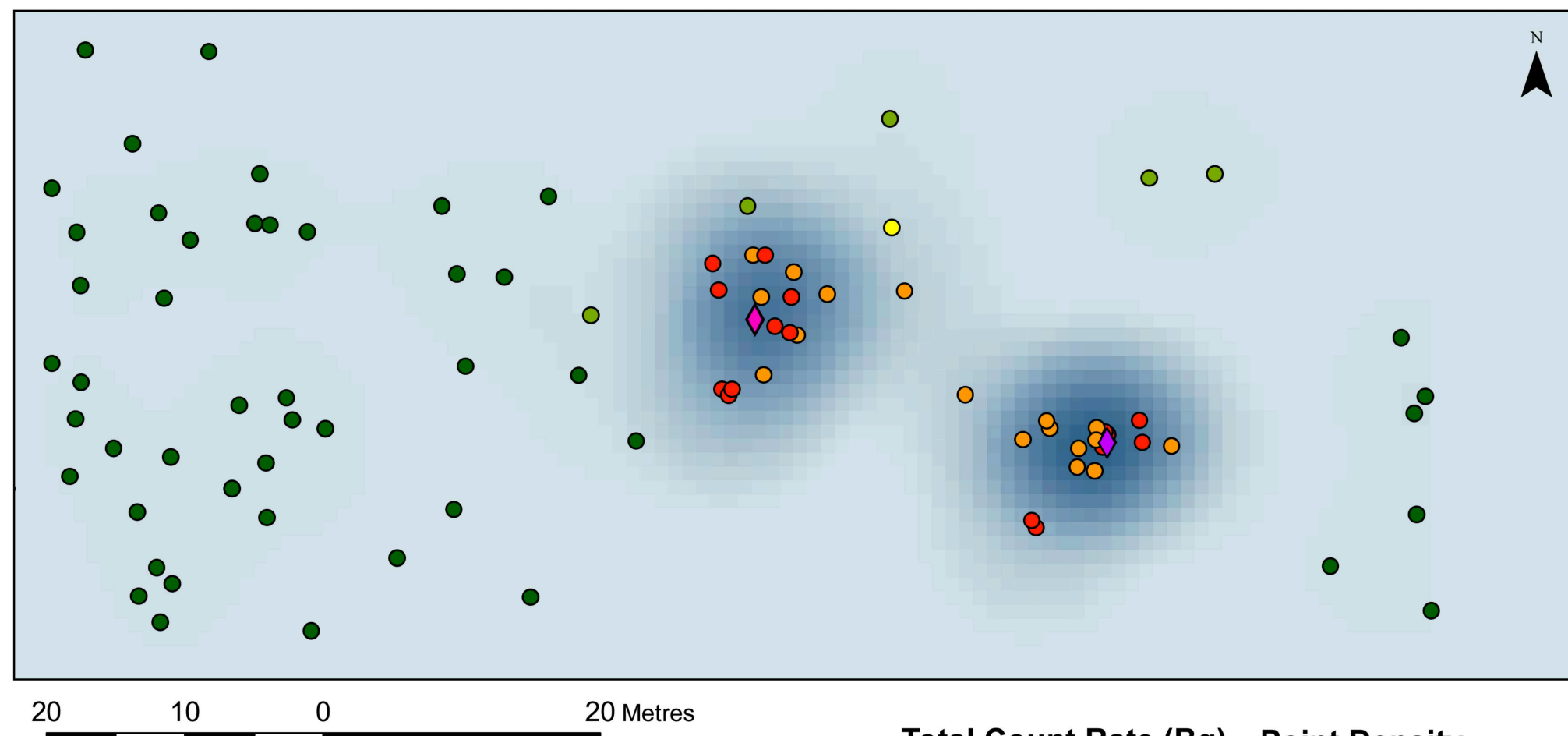

Total Count Rate (Bq) Point Density

- $26-250$

○ $251-500$

○ $501-750$

○ $751-1000$

Low : 0

- $1001-1250$

Source Location

\section{$\diamond$ PYL1 \\ $\checkmark$ PYL2}

Figure 4.9: Projection map of the survey over two point sources. The direction vector intersection points are shown by the colored circles. Red indicates a high total count rate, while green indicates a low total count rate. The intersection point density is indicated by the blue map, where a darker blue indicates a higher density. The true source locations are indicated by the pink and purple diamonds. 
altitude from the autopilot, the points of intersection of the projected downwardpointing direction vectors were calculated and are shown where they intersect the earth's surface. The intersection points are weighted by the number of gamma-ray energy deposits at the measurement point to which they correspond and this weighting is indicated by the colour of the intersection point markers. The resulting blue point density contours were calculated using the algorithm described in Section 3.2, with higher densities represented by a darker blue colour. The point density contours show that there are two high density anomalies within the survey area. This indicates that there are two sources in the survey area.

\subsection{Discussion}

The results from these two surveys show that NRCan's radiometric UAS is capable of mapping and locating point sources of radiation. While natural radioactive sources are not typically found as point sources, these initial trials indicate that the radiometric UAS can be used to perform high spatial resolution surveys for mineral exploration, environmental and nuclear emergency response applications.

The ground sampling area for a gamma spectrometer can be approximated by an oval with a width of two times the flight altitude, and a length of two times the flight altitude plus the distance during the accumulation time [6]. For the flights conducted over the point sources, the sampled area per data point is an oval with dimensions of approximately $20 \mathrm{~m} \times 22 \mathrm{~m}$. This is a significantly smaller resolution when compared to the typical manned fixed wing surveys with sampling areas of $240 \mathrm{~m} \times 280 \mathrm{~m}$.

These point source trials also show how the UAS's directional information can further improve the spatial resolution and source locating capabilities. The projection map is shown to narrow the source location results when compared with the count rate map alone. 
When comparing the single source count rate map (Figure 4.5) to the single source projected map (Figure 4.6), the results from both maps indicate similar locations for the source, which aligns well with the true source location. This was expected for the single source trial. There is a notable reduction in the spatial extent of the projection map when compared with the count rate map. While the maximum values of the two maps are in the same location, the extent of the anomaly in the projection map is limited to a diameter of approximately $15 \mathrm{~m}$. The anomaly produced in the count rate map has a larger extent, with a diameter of approximately $30 \mathrm{~m}$, before reaching a background count rate.

The narrowing of the spatial extent for the source locations becomes more relevant in the case of two point sources. The reduction in the spatial extent of the anomaly become more pronounced for two point sources, as the projection map is shown to distinctly identify both point sources, while the count rate map shows an elongated anomaly that could be resulting from a distributed source.

This reduced spatial extent and improved source locating can benefit radiometric surveying applications. For mineral exploration, the results from the radiometric UAS could provide better knowledge of ore deposit geometry and guide further geophysical, geochemical, or drilling investigations. The directional capabilities can also benefit environmental remediation applications. The UAS can provide a more precise knowledge of where the contamination is located, and therefore provide targeted information for where remediation efforts would be the most beneficial.

In addition to reducing the spatial extent of a source location in post-processing, the direction vectors can be displayed in real time. The information can then be used by the UAS operator to guide operations as they are occurring, using the display in Figure 2.6. The information can be used to discover additional areas of interest, or to narrow in on areas where a more detailed survey may be needed. The directional 
information can be used to update and change the planned mission if it becomes apparent that a new survey is needed to fully characterize the area of interest.

The direction vectors that are overlaid on Figure 4.8 were displayed in real time and examined by the GCS operator during the flight. The direction vectors indicated to the operator that there were likely two sources present, as the direction vectors pointed to two separate locations when the UAS was flying through the middle of the anomaly. Additional surveys could have then been conducted immediately after with higher spatial resolutions to better determine the locations of these sources, and without the delay of leaving the field and post-processing the data.

The directional information would also be useful in situations where the planned mission may not have fully covered the source of interest, or an unknown source is present outside of the planned survey area. The direction vectors would indicate in real time that a source is located outside of the survey, and additional surveys could be completed to characterize the source. Often when initially planning for a survey, information about the area can be incomplete and dated. The environment may have changed at the time of the new survey. The previously known information may also be limited to a low spatial resolution, therefore it could be missing smaller features that may be of interest. The information about the area can also be from other geological data sources which may not contain enough information to predict the locations of potential radiometric anomalies.

The ARDUO is also shown to be capable of providing an estimate of the source strength using the directional information. Further work is needed to improve the analysis for data points located further from the vertical axis of the ARDUO and for analyzing the strength of multiple point sources.

Additional studies are required to include the roll and pitch of the UAS in the analysis. A detailed error analysis could also be completed. This analysis would include the errors from the positioning and orientation of the UAS, as well as the 
errors from the direction reconstruction algorithms. These studies are outside the scope of this research thesis. 


\section{Chapter 5}

\section{Distributed Source Trial}

As the NRCan radiometric UAS performed well in detecting point sources, a distributed source trial was conducted. The trial was completed in partnership with Defense Research and Development Canada (DRDC) Suffield, and took place at an experimental location at their Research Centre in Suffield, Alberta. The results from the UAS survey over the distributed source are shown in this chapter. A discussion of the results is also presented.

\section{$5.1 \quad$ Experimental Setup}

A large-scale distributed source survey was conducted at DRDC in Suffield, Alberta in March, 2018. An area of approximately $350 \mathrm{~m} \times 170 \mathrm{~m}$ was cleared of snow. The snow removal prevented changes in the spray pattern due to melting of the snow layer throughout the week-long trial period. Two unmanned ground vehicles (UGVs) were used in the trial. Figure 5.1a shows the UGV equipped with an agricultural sprayer

while distributing the ${ }^{140} \mathrm{La}$ source. The source is sprayed as a liquid from the series of nozzles along the boom at the back of the UGV. The liquid is dyed blue to visualize the spray. Figure 5.1b shows the second UGV that is equipped with a manipulator 


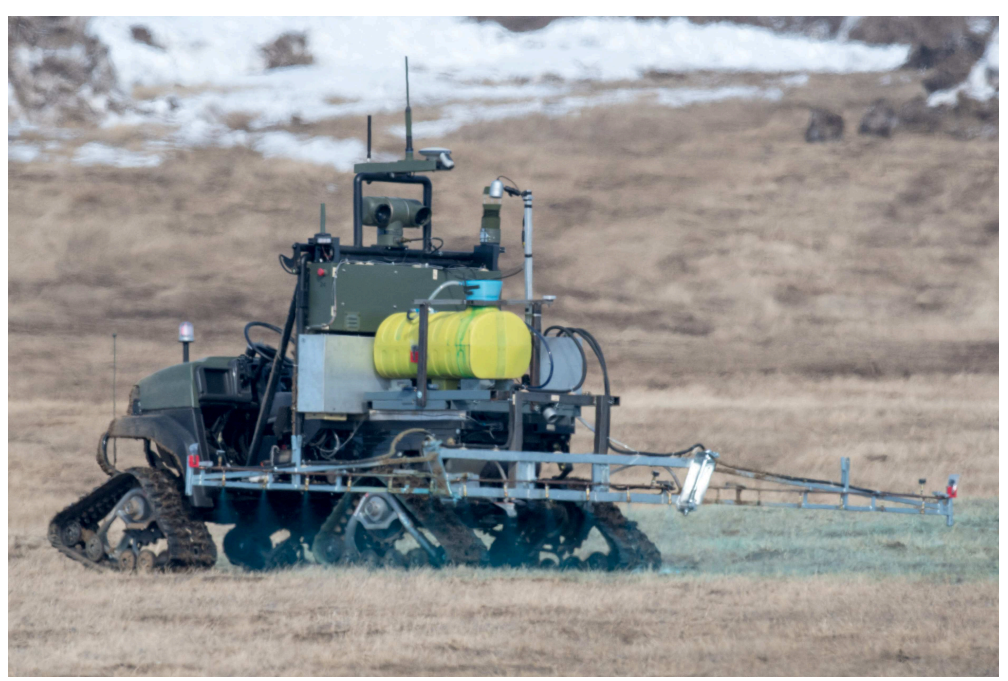

(a)

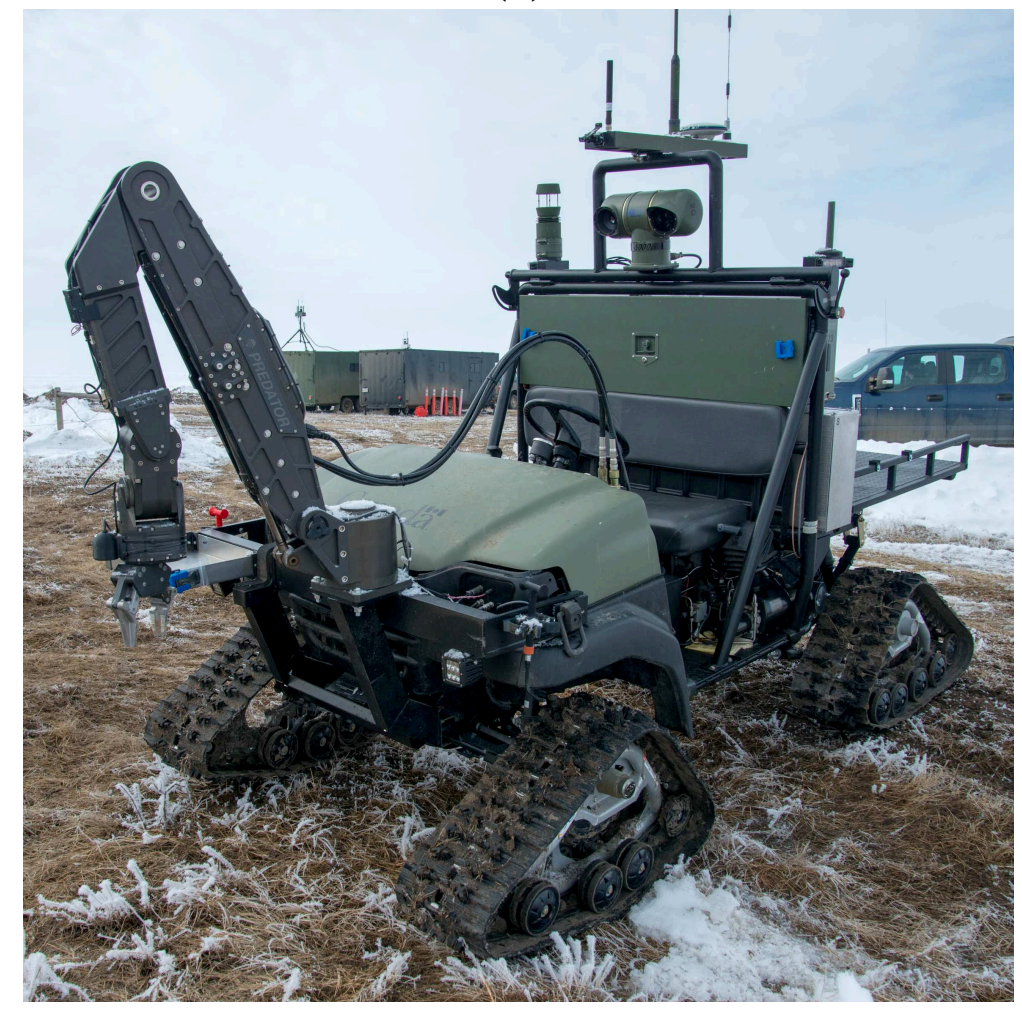

(b)

Figure 5.1: The UGVs used in the Suffield distributed source trial. The UGVs can sit two people on the front bench. a) UGV equipped with an agricultural sprayer for distributing the ${ }^{140} \mathrm{La}$ source. The UGV is in the process of distributing the ${ }^{140} \mathrm{La}$ in a solution that has been dyed blue. b) UGV equipped with a manipulator arm on the front of the UGV for transferring the source from the lead container to the sprayer UGV's tank. Photo taken by Mike Franz, DRDC Suffield Research Centre. 
arm. This UGV was used to remove the source from its lead transportation container and place it in the sprayer UGV's tank filled with the spraying liquid.

At 8:00 MDT on March 20th, 2018 the ${ }^{140}$ La source was delivered with an activity of $40 \mathrm{GBq}$. The ${ }^{140} \mathrm{La}$ was dissolved in solution in the tank on the sprayer UGV. The solution was dyed blue for visual observance of the spray pattern. On March 20th 2018 at 15:07 MT, the sprayer UGV distributed an 'L'-shaped pattern of ${ }^{140} \mathrm{La}$ with dimensions of $120 \mathrm{~m} \times 20 \mathrm{~m}$ for the long arm trending northeast-southwest, and $80 \mathrm{~m} \mathrm{x} 10 \mathrm{~m}$ for the short arm trending northwest-southeast. The activity distribution was planned to be approximately uniform at $10 \mathrm{MBq} / \mathrm{m}^{2}$ within the spray area. The sprayer was not spraying during the turning sections outside of the 'L'-shape to prevent deformation of the ' $\mathrm{L}$ '-shape at the ends of the pattern. During the spraying, the right arm of the sprayer boom became partially clogged with ice, resulting in an approximate $1 / 3$ reduction in the spray on the right side of the sprayer UGV. The reduction in the spray caused less of the ${ }^{140} \mathrm{La}$ solution to be distributed along the sprayer path than the originally planned amount. Changes to the originally planned spray pattern were therefore made to try to empty the sprayer UGV's tank and distribute all the ${ }^{140} \mathrm{La}$ solution. Two lines on the north-eastern side of the short arm of the ' $L$ '-shape were sprayed, and five lines along the long arm of the ' $L$ '-shape were sprayed. The sprayer UGV was then driven to a distance of approximately $114 \mathrm{~m}$ away from the distributed source to avoid interference with the UAS survey. A detailed description about the experimental setup can be found in Beckman et al. [10].

Figure 5.2 shows the survey area. The map has been rotated for reference with the survey area. The trial zone is marked by a fence, where only personnel with the proper personal protective equipment were allowed to enter. The UAS ground station and equipment was located within the work area along the outside of the trial zone. The UAS takeoff and landing location was approximately $10 \mathrm{~m}$ on the inside of 
the fenced trial area by the work area. The GPS path of the sprayer UGV is shown as an approximation of the location of the source distribution. The spraying was stopped periodically when the sprayer UGV reached either end of the short and long arm of the 'L'-shape, where the sprayer UGV was turning around. The two UGVs were parked to the left of the distribution. As they were contaminated by the liquid containing the source, they were parked outside of the survey area to prevent any effect from their contamination on the survey. The planned flight path is also shown in Figure 5.2 covering an area around the distributed source.

At 17:13 MT on March 20th 2018, approximately two hours after the distribution of the source, NRCan's radiometric UAS flew over the source [10]. The survey covered an area of $250 \mathrm{~m} \times 200 \mathrm{~m}$. The survey area was relatively flat. The flight was conducted at a nominal altitude of $10 \mathrm{~m}$, a nominal speed of $10 \mathrm{~m} / \mathrm{s}$ and a line spacing of $10 \mathrm{~m}$. The flight lines are shown in Figure 5.2. The UAS takeoff and landing point was placed on the inside edge of the safety zone set around the distributed source. The GCS was located on the outside edge of the safety zone. The temperature was approximately $0^{\circ}$ with low wind speeds. The flight time was $15 \mathrm{~min}$.

The spectrum of ${ }^{140} \mathrm{La}$ is complex containing several photopeaks. As the lower energy photopeaks are affected by Compton scattering, the highest energy photopeak was used. This photopeak has an energy of $1.596 \mathrm{MeV}$. It also has a high branching ratio of $95 \%$, therefore a significant amount of gamma rays with this energy are produced for each disintegration.

A background survey using the same flight plan was conducted on March 19th 2018, the day before the distribution of the source. The surficial geology of the survey area is composed of glaciolacustrine silt and clay, with minor sand [45]. There is an expected background signal from ${ }^{40} \mathrm{~K}$, and no significant contribution from ${ }^{238} \mathrm{U}$ and ${ }^{232} \mathrm{Th}$. Figure 5.3 shows the results of the background survey as a photopeak count rate map at the ${ }^{140}$ La photopeak energy of $1.596 \mathrm{MeV}$ with a three standard deviation 


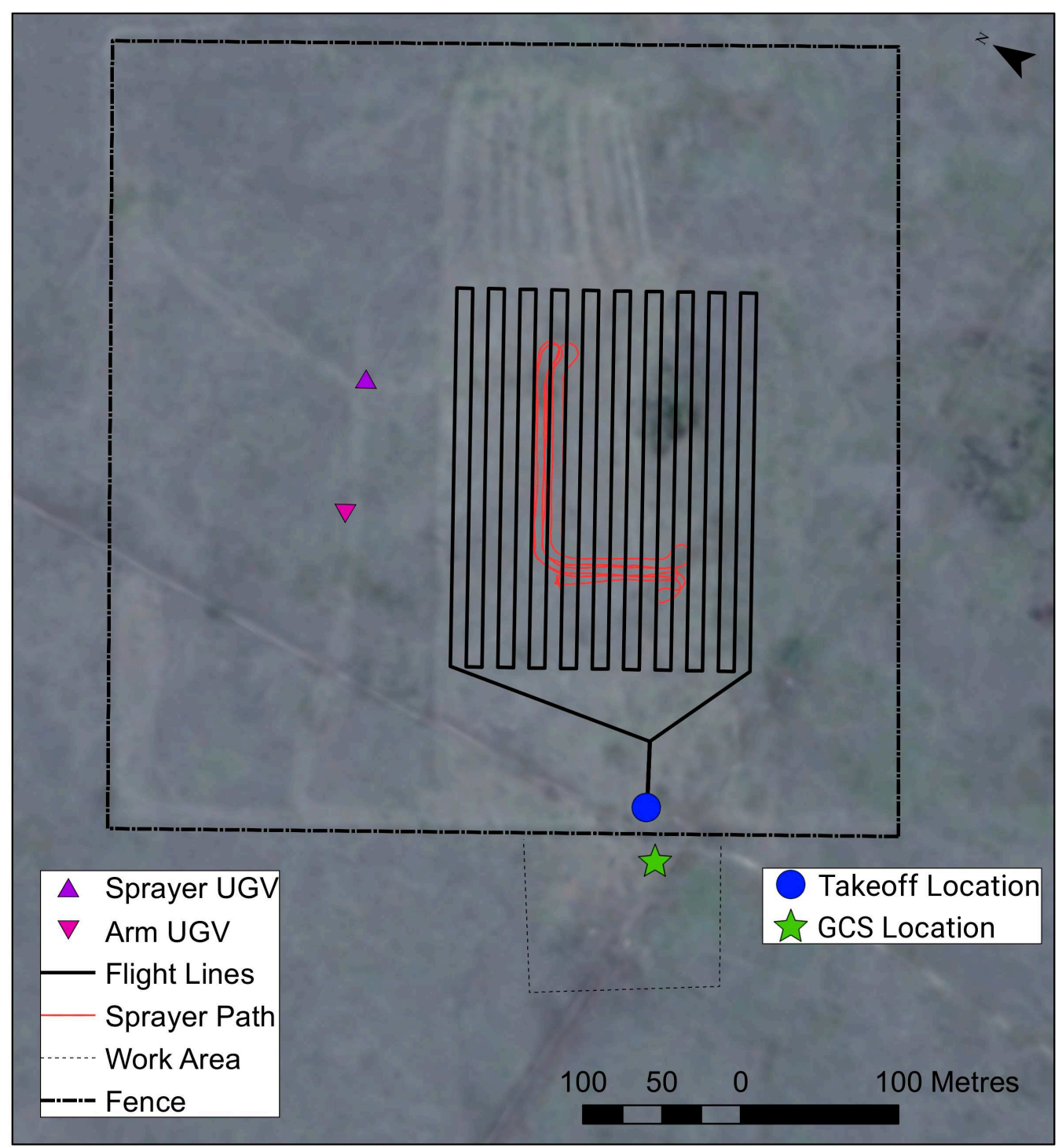

Figure 5.2: Map of the distributed source trial area. The map has been rotated for alignment with the survey area. The trial area was marked by a fence, shown by the thick dashed line. The working zone was located to the bottom right of the trial area and includes the location of the GCS, marked by the green star. The sprayer UGV path is shown by the solid red line in an 'L'-shaped pattern. The takeoff and landing location of the UAS is marked by the blue circle on the inside of the fenced trial area. The two UGVs were parked at the locations marked by the upright triangle (UGV equipped with mechanical arm) and the upside-down triangle (sprayer UGV) after the distribution of the source. The planned flight path of the UAS is shown by the thick solid black line. Imagery from Google Earth [44]. 
range around the photopeak. The direction vectors are also overlaid on the map. The map shows that there is a very low count rate throughout the survey area, with a maximum of approximately $4 \mathrm{cps}$. The count rate is approximately evenly distributed in the survey area, therefore the average background signal is used for background subtraction. The direction vectors point in random orientations, therefore indicating there is no significant localized source present within the survey area.

There was no rainfall between the time of the background survey and the survey over the distributed source, therefore background conditions are assumed to be consistent between the time of the background survey and the survey over the distributed source.

\subsection{Results}

The data from the distributed source survey and background survey were processed following the procedures described in Section 3.2. The back-decay correction explained in Section 3.2.4 was applied to the background subtracted count rate using the half-life of ${ }^{140} \mathrm{La}(144720 \mathrm{~s}$ [46]) and a reference time of 8:00 MDT on March 20th, 2018 (1521554400 s UTC). The reference time is the time of delivery of the ${ }^{140} \mathrm{La}$ source to the site.

The data, average background and background subtracted $1 \mathrm{~s}$ spectra from a representative data point over the ${ }^{140} \mathrm{La}$ source are shown in Figure 5.4. The $1 \mathrm{~s}$ back-decay corrected spectrum is not shown. The top eight histograms contain the spectra per crystal of the ARDUO. A sum of the $1 \mathrm{~s}$ spectra of the eight crystals to show the total response of the ARDUO is shown in the bottom histogram. ${ }^{140} \mathrm{La}$ is an isotope with multiple gamma emissions. The photopeak used in the analysis is shown in the signal spectra and is bound by the red dotted lines at $1.467 \mathrm{MeV}$ to 1.623 MeV. There was some gain drift in the ARDUO during the survey resulting 


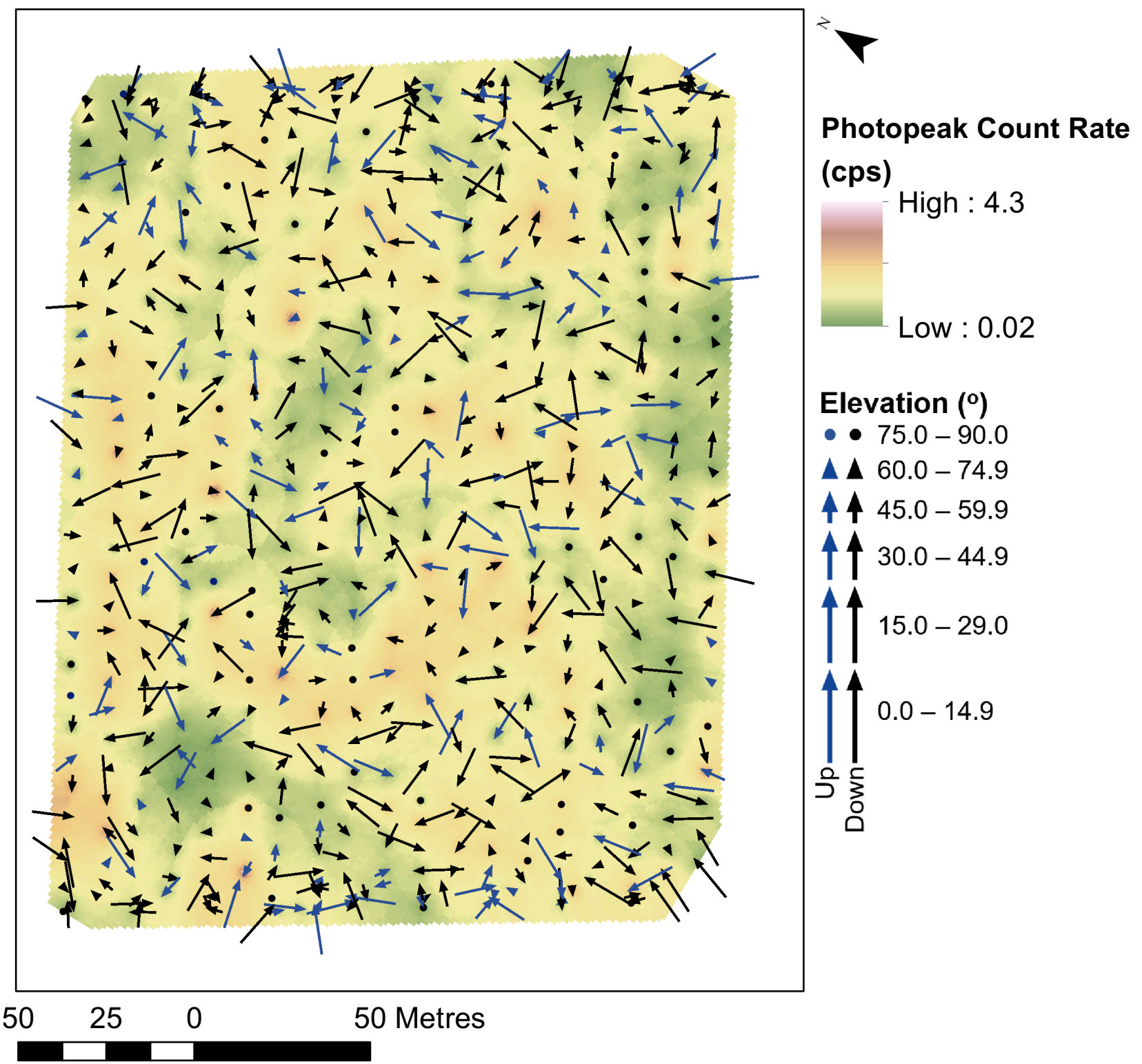

Figure 5.3: Background ${ }^{140} \mathrm{La} 1.596 \mathrm{MeV}$ photopeak map of the Suffield distributed source survey area. A low photopeak count rate is shown by a green colour and a high photopeak count rate is indicated in red. The direction vectors are overlaid with the arrowhead placed at the data point location.

in a lower output photopeak energy than the true photopeak energy of $1.596 \mathrm{MeV}$.

The photopeak bounds were centered on the output average photopeak energy with a range of 3 standard deviations. The average background count rate is significantly lower than the signal count rate, and is shown at a near zero count rate in Figure 5.4. 
Low energy noise from crystal 2 can be seen in the background spectrum. This noise is likely caused by random fluctuations in the electronics of the ARDUO and is not typical behaviour of the system. This noise does not affect the data analysis as the total count rate energy window has a lower range of $0.1 \mathrm{MeV}$. The noise seen in crystal 2 is below the $0.1 \mathrm{MeV}$ threshold. To reduce the effects of the low energy noise in the real-time display, the spectra data were collected with a low energy cutoff of approximately $0.05 \mathrm{MeV}$. This resulted in no data being collected for an energy near zero, and therefore no spectral information is displayed near zero.

The signal, background and background subtracted spectra averaged over the entire survey for crystal 1 of the ARDUO are shown in Figure 5.5. The back-decay corrected spectrum is not shown. The averaged spectra from the other ARDUO crystals are similar. The spectra are plotted on a logarithmic scale to allow comparison of the background spectrum with the significantly larger and signal spectrum. The high energy ${ }^{140} \mathrm{La}$ photopeak at $1.596 \mathrm{MeV}$ is shown in the signal spectra and the photopeak bounds of $1.467 \mathrm{MeV}$ to $1.623 \mathrm{MeV}$ are shown by the dotted red lines. The background spectrum is shown by the solid green spectrum. The $1.460 \mathrm{MeV}$ photopeak from ${ }^{40} \mathrm{~K}$ is shown in the background spectrum, close to the ${ }^{140} \mathrm{La}$ photopeak range used in the analysis. This background signal is subtracted from the data to reduce the effect from this photopeak. As the signal count rate is also approximately 100 times higher than the average background count rate, the background has minimal effect on the measured signal.

The back-decayed photopeak count rate map is shown in Figure 5.6. The direction vectors of each data point are overlaid as well as the sprayer UGV path. There is a high photopeak count rate along the long arm of the 'L'-shape, corresponding well with the sprayer path. The short arm of the ' $L$ '-shape shows a lower count rate. This corresponds to the lower number of lines sprayed along the short arm. The signal 

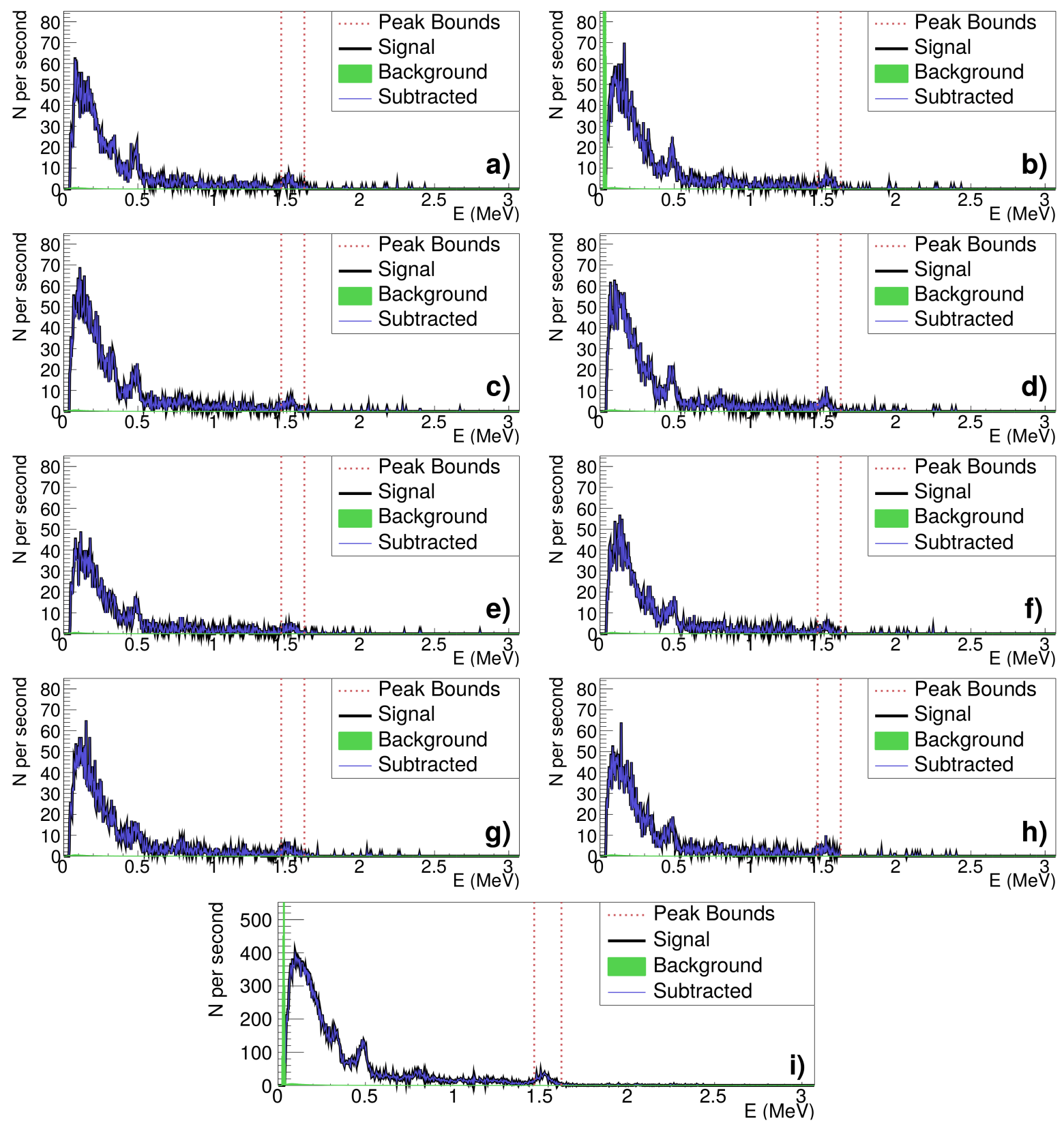

Figure 5.4: Representative $1 \mathrm{~s}$ energy spectra for a data point located over the ${ }^{140} \mathrm{La}$ source for the distributed source survey. The y-axis is the number of counts, $\mathrm{N}$, per second. The $\mathrm{x}$-axis is the energy, $\mathrm{E}$, in $\mathrm{MeV}$. The $1 \mathrm{~s}$ signal spectrum is shown by the black line. The average background energy spectrum for the survey area is shown by the green-filled area. The signal with the average background subtracted is shown by the blue line. The energy window bounds used for the photopeak analysis are shown by the red dotted lines. a) to h) Representative $1 \mathrm{~s}$ energy spectra from each of the eight ARDUO crystals, 1 to 8 respectively. i) Total $1 \mathrm{~s}$ spectra from the sum of the eight crystals. 


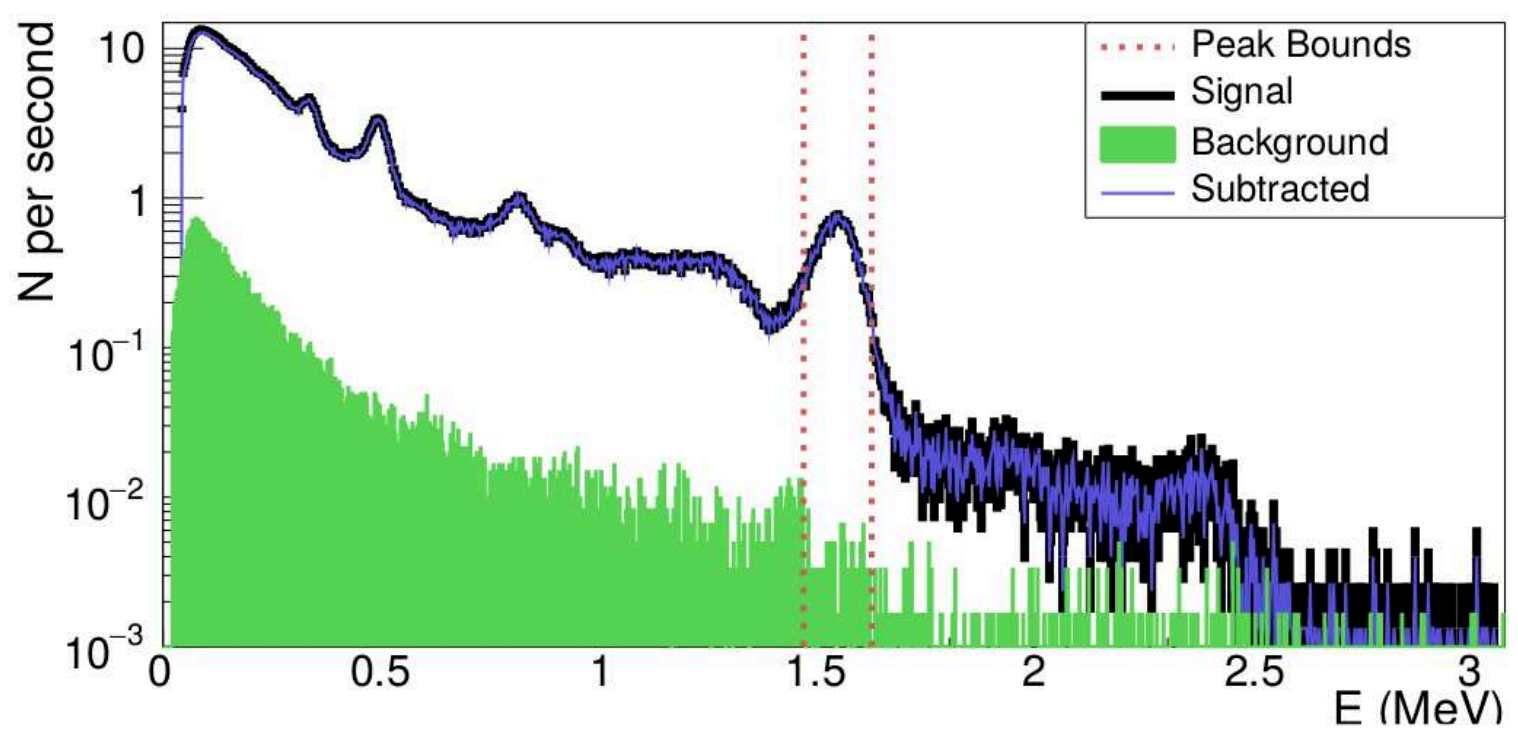

Figure 5.5: Energy spectra from crystal 1 of the ARDUO while flying over the distributed source of ${ }^{140} \mathrm{La}$. The $\mathrm{y}$-axis is the number of counts, N, per second. The $\mathrm{x}$-axis is the energy, $\mathrm{E}$, in $\mathrm{MeV}$. The $1 \mathrm{~s}$ raw data spectrum is shown by the black line. The average background energy spectrum for the survey area is shown by the green-filled area. The background subtracted data spectrum is shown by the blue line. The energy window bounds used for the photopeak analysis are shown by the red dotted lines.

from the short arm is partially masked by the higher count rate of the long arm, and only becomes distinctive at a distance of approximately $25 \mathrm{~m}$ from the right edge of the long arm. The extent of the anomaly to a count rate of $300 \mathrm{cps}$ is approximately $100 \mathrm{~m} \times 170 \mathrm{~m}$ for the long arm. The extent of the short arm is difficult to distinguish from the long arm's count rate. The short arm does cause an extension of the anomaly approximately $25 \mathrm{~m}$ further to the right side of the long arm along the bottom edge of the long arm. The survey area is not large enough to reach a far enough distance away from the source to measure background levels. The lowest measured count rate of $16 \mathrm{cps}$ is $4 \times$ the maximum background count rate.

The overlaid direction vectors provide a good indication as to where the source is located. They also continue to point towards the source at data point locations near the edges of the survey area, further indicating that background levels have not been reached in the survey area. As the direction vectors approach the source they point 
along the length of the source distribution, indicating that the signal is not caused by a point source. The vectors also become more vertical in the downward direction as they approach both arms of the 'L'-shape. The direction vectors are evidence that there is a portion of the source located in the short arm, which is less evident in the count rate map. The near-vertical direction vectors occur in both the short and long arm of the ' $L$ '-shape.

In addition to the count rate map, the back-decayed count rates can be used to calculate a surface activity concentration, as described in Section 3.2.10. This thesis presents a preliminary analysis of the surface activity concentration. A detailed comparison between the Monte Carlo simulations and the data from the ARDUO is required to determine the validity of the simulated sensitivity value. As well, a detailed analysis of the errors associated with the position and orientation of the UAS during each measurement is required. Additionally, a change in altitude can cause a significant change in the measured count rate and therefore the calculated surface activity concentration.

The distribution in the altitude measurements is shown in Figure 5.7. The $0.3 \mathrm{~m}$ offset from the autopilot to the center of the ARDUO has been subtracted from all altitude measurements. The range of accepted altitudes was determined using the process described in Section 3.2.6. The altitude range bounds correspond to values of $9.11 \mathrm{~m}$ and $10.07 \mathrm{~m}$. Data points with altitudes within this range were included in the analysis, while data points with altitudes outside of this range were excluded. The accepted altitude range is centered around the mean altitude of $9.59 \mathrm{~m}$. The majority of the stochastic fluctuations in the altitude are within this range. During the UAS flight over the distributed source, it was observed that the UAS had some difficulty maintaining a nominal altitude of $10 \mathrm{~m}$ and would occasionally drop in altitude. This is reflected in the altitude distribution by a minor skew towards the lower altitudes. Additionally, there may be a systematic offset of the altitude. 


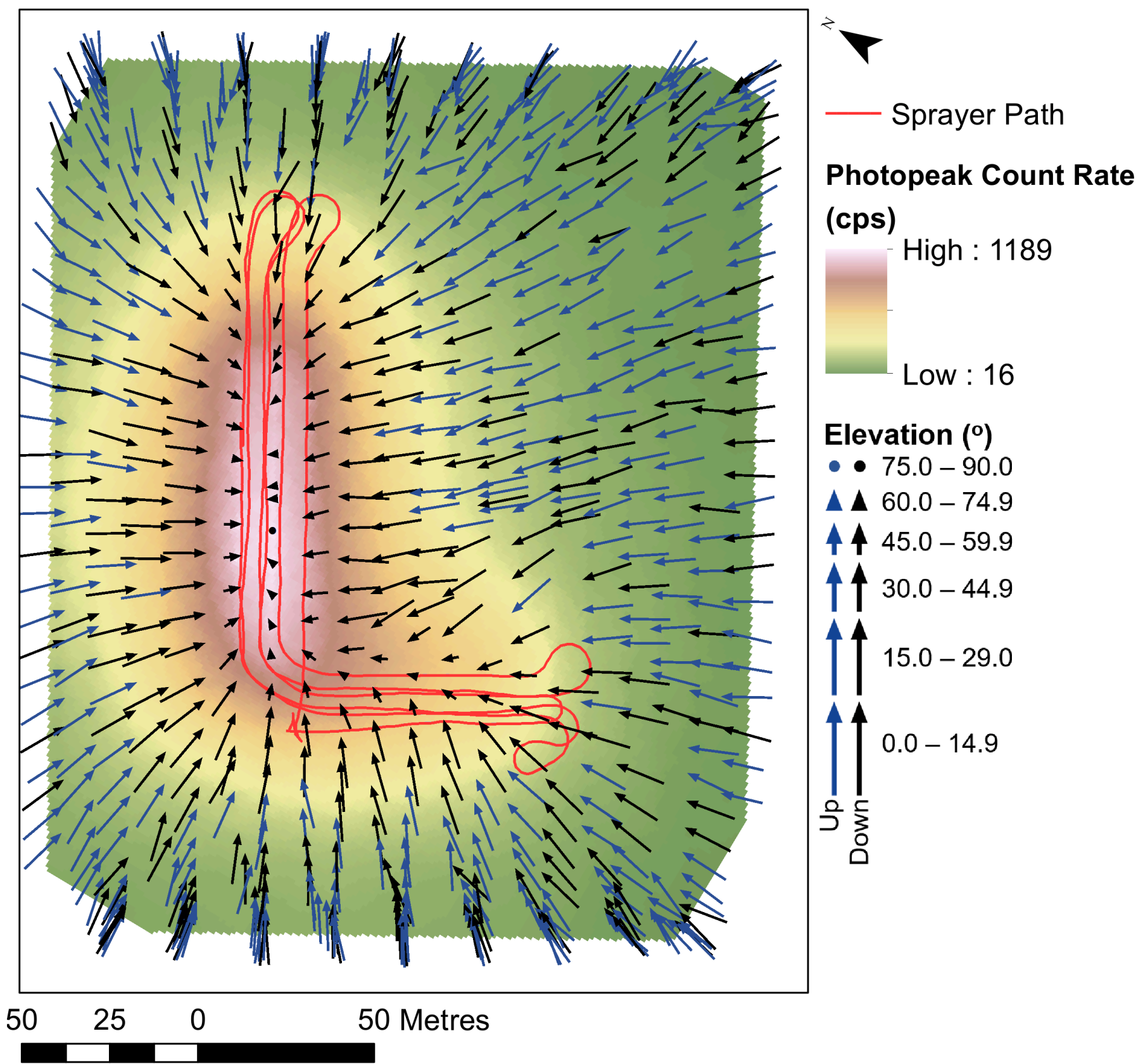

Figure 5.6: Back-decayed photopeak count rate map of the ' $L$ '-shaped distribution of ${ }^{140} \mathrm{La}$. A high count rate is represented by a pink colour, and a low count rate is represented by a green colour. The direction vector from each data point is overlaid, with the arrowhead at the location of the data point. The sprayer UGV path is shown by the red line. 


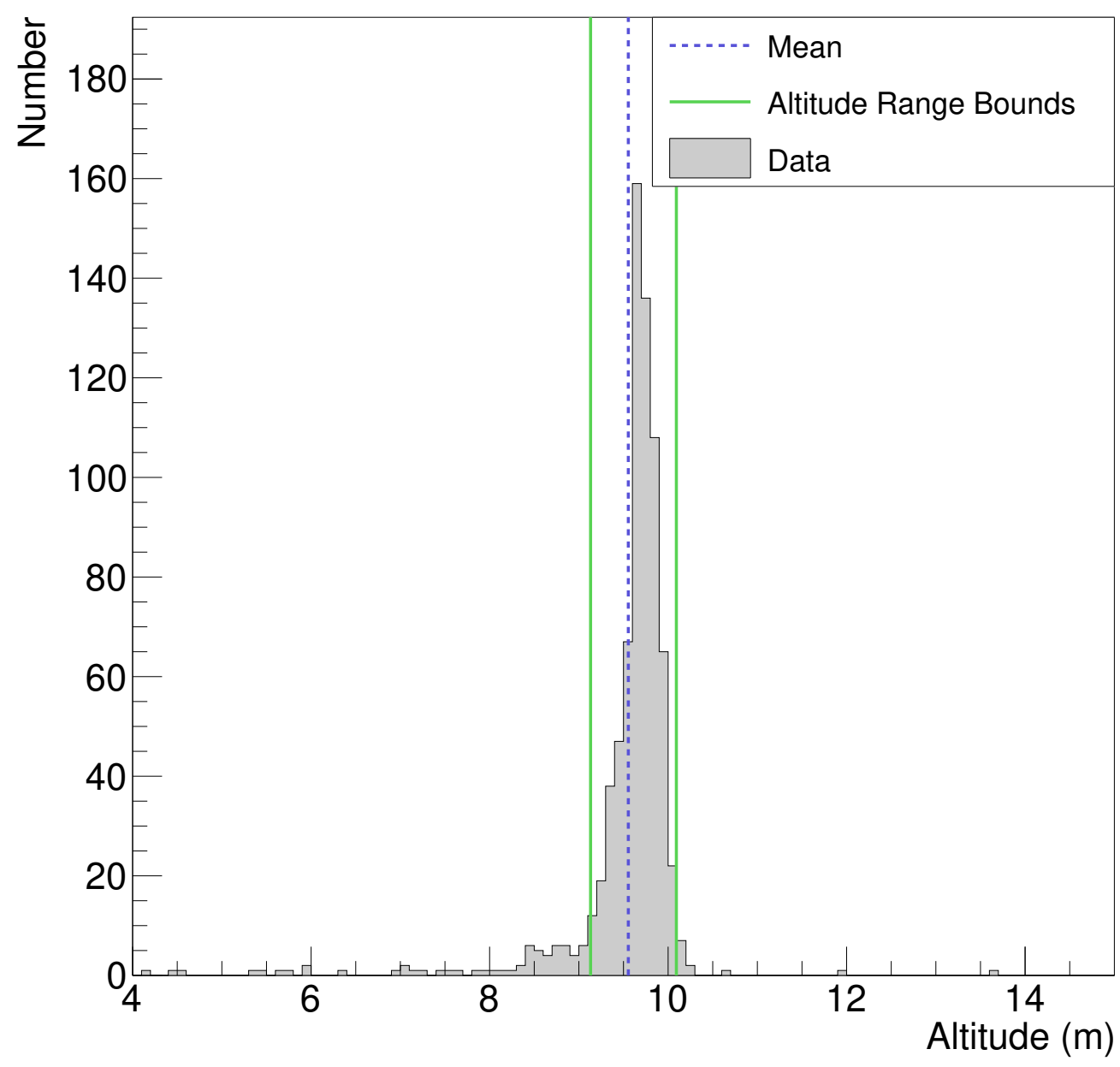

Figure 5.7: Histogram of the altitude measurements for the distributed source trial. The mean of the altitude distribution is shown by the blue dashed line. The altitude range boundaries that correspond to a $10 \%$ change in the count rate due to a change in altitude are shown by the solid green lines.

Through visual observations of the UAS in flight, the systematic error is estimated to be $1 \mathrm{~m}$.

The preliminary map of the surface activity concentration for the distributed source trial is shown in Figure 5.8. The direction vectors are overlaid on the map and point along the whole distribution of the source. The sprayer UGV path is also overlaid to show an approximation of the true location of the source distribution. The surface activity distribution in the area is the same as the count rate distribution 
exhibited in the photopeak count rate map with the only difference being the colour scale values.

The maps of the count rate and surface activity concentration show that the measured values attributed to the distributed ' $L$ '-shaped source extend beyond the true boundaries of the distribution. The extension of the radiometric anomaly beyond the true extent of the source is due to the field-of-view of the ARDUO. For sources assumed to be located on the ground, the field-of-view can also be defined as the ground sampling area. The ground sampling area is dependent on the altitude and speed of the ARDUO. The measured count rate, and therefore the measured surface activity concentration, is resultant from all radioactivity within this ground sampling area. For ARDUO data point locations beyond the true extent of the distributed source, the ARDUO continues to measure a signal from the source. This causes a smearing of the source signal in the count rate and surface activity concentration maps, where the signal from the source is underestimated directly above source, and over-estimated at locations further from the source.

The effect of the smearing is best shown by the surface activity concentration map. The maximum calculated surface activity concentration is approximately 1 $\mathrm{MBq} / \mathrm{m}^{2}$, located in the long arm of the ' $\mathrm{L}$ '-shape. This is lower than the intended surface activity concentration of $10 \mathrm{MBq} / \mathrm{m}^{2}$ by an approximate factor of 10 . This may be due to a number of effects, one of which is the smearing effect described above. A new method has been developed by Sinclair and Fortin [47] to deconvolve the spatial smearing of the data, therefore producing a more precise result of the spatial distribution of the source. The use of this method to improve the surface activity concentration reconstruction results is outside the scope of this thesis.

By integrating the surface activity concentration anomaly caused by the source over the total area of the anomaly, an estimate of the total activity of the source within the area can be calculated. A tentative conclusion based on a comparison of 
the measured integrated source activity with the intended true source activity can then be made.

The surface activity concentration map for the distributed source was summed over the total survey area at a grid spacing of $1 \mathrm{~m}^{2}$, resulting in a total activity in the survey area of $12 \mathrm{GBq} \pm 1 \mathrm{GBq}$. The error was calculated allowing for an uncertainty in the altitude of $1 \mathrm{~m}$ from stochastic fluctuations and potential systematic bias. The uncertainty due to the simulated ARDUO response having a poor correspondence to the true ARDUO response could be large and is unknown.

A source strength estimation using the direction vectors is outside the scope of this thesis. The analysis is more complex for a distributed source than for the simplified analysis conducted for the single point source survey. The spatial deconvolution method that can be used to reconstruct the source strength of a distributed source using a directional spectrometer is described in the Masters thesis by Marshall [48].

The projection map for the survey over the distributed source is shown in Figure 5.9. The intersection points that are closer to vertical in the downwards direction, less than $-40^{\circ}$, were used for the projection map. The points were also weighted based on the total count rate of the data point at its original location. The sprayer path is overlaid as an indication of the true source distribution. The point density shows a high anomaly along the long arm of the 'L'-shaped distribution as well as along the short arm. The short arm is located towards the top section of the sprayer path as this is where most of the spraying in the short arm occurred along the top lines of the sprayer path. The long arm corresponds well to the sprayer path. The point density anomaly has an extent of approximately $30 \mathrm{~m} \times 115 \mathrm{~m}$ for the long arm and $70 \mathrm{~m} \times 25 \mathrm{~m}$ for the short arm. This spatial extent is much smaller than the extent shown in the count rate map where count rates over $100 \mathrm{~m}$ from the maximum are above background levels. 




Figure 5.8: Surface activity map of the 'L'-shaped distribution of ${ }^{140} \mathrm{La}$. A high surface activity is represented by a pink colour, and a low surface activity is represented by a green colour. The direction vector from each data point is overlaid, with the arrowhead at the location of the data point. The sprayer $\mathrm{UGV}$ path is shown by the red line.

The projection method, enabled by the direction vector reconstruction capabilities of the ARDUO, is also able to clearly determine the presence of the short arm of the 
'L'-shape, where the count rate map was unable to provide conclusive evidence of the existence of the short arm. The point density anomaly is also higher in the long arm than in the short arm, corresponding to the higher surface activity concentrations in the long arm and lower concentrations in the short arm.

\subsection{Discussion}

The results from this trial show that the radiometric UAS is capable of performing larger scale surveys at low altitude and slow speeds. The UAS was able to survey an area of $250 \mathrm{~m} \times 200 \mathrm{~m}$ in 15 minutes. The count rate and surface activity concentration maps show the anomaly caused by the distributed source. The anomaly extends beyond the survey area as background levels are not reached at the edges of the survey boundaries. The UAS defines well the source in the long arm of the 'L'shape using the count rate and surface activity concentration maps. The source is known to have a higher activity in this arm due to the extra lines that were sprayed along this section. The short arm of the 'L'-shape is less defined, but shows some extension to the right of the long arm. The lower activity in the short arm is partially masked by the high activity concentration signal from the long arm.

The overlaid direction vectors on the count rate and surface activity concentration maps point towards the distributed source. As the signal from the source is approximately $4 \times$ background levels at the limits of the survey area, the direction vectors still point towards the distributed source. At the limits of the survey area, the azimuth angle is well reconstructed, while the elevation angle is near horizontal in the upwards and downwards directions. This is expected as the ARDUO is less capable of reconstructing elevation angles near the horizon. As the direction vectors approach the source the elevation becomes more indicative of where the source is located. The 


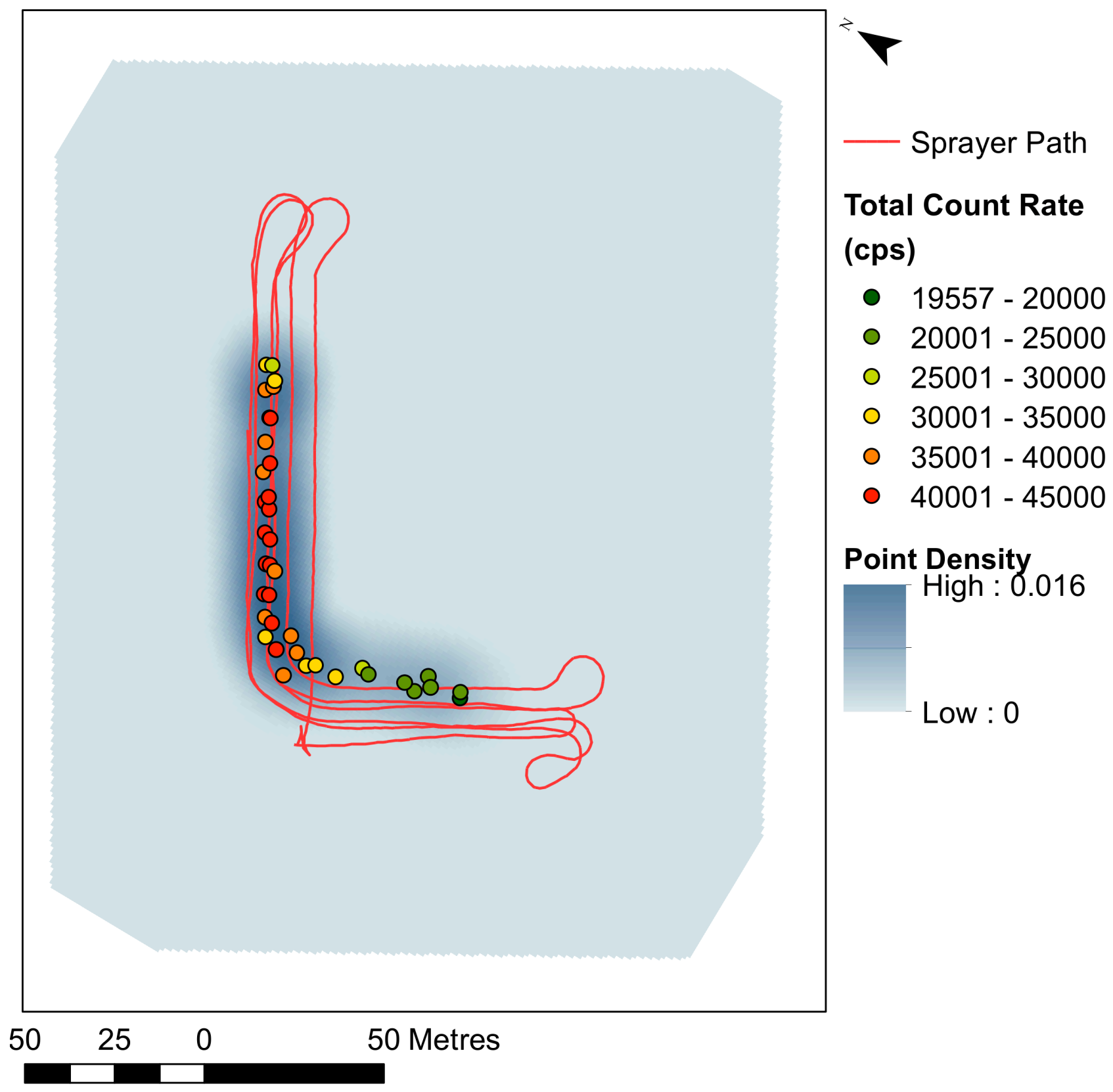

Figure 5.9: Projection map of the ' $\mathrm{L}$ '-shaped distribution of ${ }^{140} \mathrm{La}$. A high point density is shown by a dark blue colour, and a low point density is shown by the light blue colour. The intersection points of the direction vectors with the ground are shown by the circles. The colours of the circles correspond to the total count rate of the original data point, with red indicating a high total count rate and green indicating a low total count rate. The red line shows the sprayer UGV path. 
direction vectors located directly over the source have near-vertical reconstructed elevation angles. This is shown for both the high activity concentration long arm of the ' $\mathrm{L}$ '-shape as well as for the lower activity concentration of the short arm. As well, the direction vectors point along the length of both arms of the ' $\mathrm{L}$ '-shape, indicating that the source is distributed along an area rather than being the result of several point sources.

When the direction vectors are projected to the ground's surface, the area of the source location is much smaller than the extent of the count rate anomaly. The short arm also becomes more distinct, with a narrower extent and indicating that the source is truly distributed in an 'L'-shape. When compared to the planned dimensions of the ' $\mathrm{L}$-shape, the point density anomaly's extent is similar. The point density dimensions are within $5 \mathrm{~m}$ to $15 \mathrm{~m}$ of the planned dimensions, with the largest difference present in the width of the short arm. This indicates that the projection method is capable of correctly narrowing the source location. An error analysis has not been completed for this method. For a preliminary assessment, the results are promising for the method's ability to reconstruct the true source distribution.

There are no intersection points that are located off of the 'L'-shape. This is because a background count rate was not reached in the survey area. All the direction vectors were reconstructed towards the source, with the further data point locations reconstructing near the horizon. The near-horizon direction vectors are not used in the analysis due to their poor elevation angle reconstruction.

The surface activity concentration map was produced to estimate the total amount of activity in the area. As the surface activity concentration assumes an infinite sheet of radiation, the calculated value is underestimated in the area over the source, and over estimated in the area where no source is present. By integrating the surface activity concentration over the full area of the anomaly caused by the source, the total activity of the source can be calculated. The total activity calculated for the 
survey was $12 \mathrm{GBq} \pm 1 \mathrm{GBq}$, neglecting the uncertainty associated with a possible misrepresentation of the ARDUO and the experimental conditions in the simulated model used to calculate the sensitivity. As this value was back decayed to the time of delivery of the ${ }^{140} \mathrm{La}$ source, the true total activity is $40 \mathrm{GBq}$. A lower value for the calculated total activity is expected as the UAS survey did not fully capture to total area of the anomaly caused by the source as background levels were not reached. As well, some amount of the ${ }^{140} \mathrm{La}$ was residual in the sprayer tank and spray arms, that may contribute to the loss of activity in the 'L'-shaped distribution. Additionally, a validation of the Monte Carlo simulation data used to determine a sensitivity value is required to confirm that the sensitivity value used is correct for the ARDUO. As a preliminary examination of the total activity that was distributed, the calculated $12 \mathrm{GBq} \pm 1 \mathrm{GBq}$ is within the same magnitude of the true activity and is a reasonably consistent with expectations.

These results show that the UAS is capable of mapping and locating distributed sources, similar to those that would be of geophysical interest for applications in mineral exploration, environmental applications and security applications. The higher spatial resolution maps can result in better targeting of areas that may require further investigations. The UAS's direction vector projection analysis can significantly improve these targeting efforts by providing better information on the true source location. The same method was used for both point sources and distributed sources, therefore indicating that the method is functional for various source geometries.

The overlaid direction vectors in Figure 5.6 show that the directional information is useful at a distance up to $130 \mathrm{~m}$ away from the source at count rates of approximately $4 \times$ background levels. While the detection distance varies depending on the source strength, this trial exhibits the use of the direction vector information in postprocessing, as well as for a real-time display. If only one line was flown along the right side of the 'L'-shape, the direction vectors would show that there is a significant 
source located towards the left of the survey line, requiring further investigations. Changes to the survey could then be completed in real time to better capture the detected source. 


\section{Chapter 6}

\section{Summary and Conclusions}

NRCan designed a radiometric UAS that uses the novel directional gamma spectrometer called the ARDUO mounted on a helicopter airframe. The radiometric UAS was designed to conduct high spatial resolution radiometric surveys for geophysical exploration, environmental applications, and for nuclear emergency response. The performance of the radiometric UAS was demonstrated through a series of laboratory experiments, small-scale surveys, and a large-scale survey.

With regards to the first objective in Section 1.2, laboratory tests were conducted to characterize the response of the ARDUO. The FWHM energy resolution for each of the crystals is approximately $7 \%$, which is within the acceptable operational limit for radiometric surveying. The ARDUO exhibits good linearity over a range of energies, which is desirable for a spectrometer. The gain drift was examined and used to guide operational procedures. The intrinsic photopeak efficiency for a point source of ${ }^{137} \mathrm{Cs}$ located directly below the ARDUO was determined to be $32.0 \% \pm 0.01 \%$. The intrinsic photopeak efficiency was then used to analyze the ability of the UAS to re-

construct the source strength for the single ${ }^{137} \mathrm{Cs}$ point source survey. The directional response was also characterized. The results indicated that the ARDUO performs well in reconstructing azimuth angles. The elevation angles are reconstructed well at near vertical angles, but elevation angles near the horizon are poorly reconstructed. 
This is due to the rectangular geometry of the crystals of the ARDUO, as exhibited by the simulated elevation angle response. The study of the ARDUO's direction reconstruction ability was used to determine the elevation angle cutoff for the direction vector projection method.

In the small-scale point source trials the UAS demonstrated it is capable of producing high spatial resolution maps of $110 \mathrm{~m} \times 50 \mathrm{~m}$ area through a survey at an altitude of $10 \mathrm{~m}$, speed of $2 \mathrm{~m} / \mathrm{s}$ and line spacing of $4 \mathrm{~m}$. The direction information from the ARDUO is shown to improve the source locating abilities of the UAS. This was distinctly demonstrated by the two point source trial, where the projection map is able to distinguish the two sources at a separation of $27 \mathrm{~m}$. Furthermore, an initial assessment of the ability of the system to determine the source strength was completed. This resulted in a source strength of $175 \mathrm{MBq} \pm 39 \mathrm{MBq}$. The true source strength of $161 \mathrm{MBq}$ is within error of this result, indicating that this method could be used for determining the source strength.

A final trial was completed over a large-scale area of $250 \mathrm{~m} \times 200 \mathrm{~m}$ containing an 'L'-shaped distributed source in Suffield, Alberta. The 'L'-shaped source distribution had dimensions of $120 \mathrm{~m} \times 20 \mathrm{~m}$ for the long arm and dimensions of $80 \mathrm{~m} \times 10 \mathrm{~m}$ for the short arm. The UAS was able to produce a high spatial resolution count rate map through a survey at an altitude of $10 \mathrm{~m}$, speed of $10 \mathrm{~m} / \mathrm{s}$ and line spacing of $10 \mathrm{~m}$. The sensitivity of the ARDUO to a large sheet source as a function of altitude was then determined by Dr. Andrew McCann using Monte Carlo simulations for a photopeak energy of $1.596 \mathrm{MeV}$. The sensitivity allowed a preliminary surface activity concentration map to be produced. Similar to the results of the point source trials, the results from the distributed source trial showed that the UAS is capable of producing high spatial resolution radiation maps using its directional gamma spectrometer. The direction information was used to produce a projection map, where the extent of the distributed source is narrowed and closer to the known true source outline when 
compared to the photopeak count rate map. The projection map was shown to be capable of locating the lower activity of the short arm of the ' $\mathrm{L}$ '-shape. The count rate map was less capable of distinguishing the signal from the short arm as it was partially masked by the high count rate signal from the long arm of the ' $L$ '-shape. The total activity attributed to the distributed source was estimated to be $12 \mathrm{GBq} \pm 1 \mathrm{GBq}$. This is reasonable given the intended true total activity of $40 \mathrm{GBq}$. The difference between the total activity values could be caused by a few factors. The extent of the survey area did not fully capture the complete extent of the anomaly caused by the distributed source. A validation of the sensitivity function obtained through Monte Carlo simulations with real data has not been completed. As well, some of the ${ }^{140} \mathrm{La}$ source was residual in the sprayer UGV's equipment after spraying the 'L'-shape.

The results from the point source and distributed source trials with the UAS demonstrate the surveying capabilities of the UAS, fulfilling the second objective stated in Section 1.2. The UAS was shown to be capable of completing high spatial resolution radiometric surveys using flight parameters of an altitude of $10 \mathrm{~m}$, with line spacings of $10 \mathrm{~m}$ or less, and speeds of $10 \mathrm{~m} / \mathrm{s}$ or less. High spatial resolution maps of the count rate were made for both trials, and an additional high resolution surface activity concentration map for the distributed source trial was made. The UAS was also shown to be capable of estimating the source strength of a point source, as well as estimate the total activity distributed in the survey area.

Completing the third objective from Section 1.2, the direction projection method was shown to further improve the spatial resolution of the survey by improving source locating. The UAS was able to delineate the shape of two sources of equal strength within close proximity, as well as delineate the shape of a weak distributed source in the presence of a strong distributed source.

For geological exploration, the source location information can be used to guide further exploration efforts, such as geochemical analyses or drilling efforts. By using 
the directional information, exploration efforts can better target the source of the anomaly, potentially reducing costs to the project. As well, the detailed surficial anomaly information could provide useful information on the deposit geometry. Additionally, the direction information could be useful in targeting areas outside of the initial survey area. As initial information about the area may be dated or not well known, it may be the case where the strongest anomaly may not be included in the original surveying plan. The directions could indicate that a re-evaluation of the area may be needed by indicating that a stronger source is located outside of the survey area.

The high spatial resolution and source locating capabilities of the UAS can also be used in environmental applications to more accurately target remediation and monitoring efforts. Environmental surveys often target man-made sources which can have different source geometries when compared to geological sources. Man-made sources can be found as point sources, as well as distributed sources. They can also have greater variations in their activity and distribution over shorter distances. The directional capabilities of the UAS have been shown to delineate these source geometries where count rate maps are unable to. This information could therefore be used to reduce the area that may require remediation, and consequently the associated costs, by being able to better target the true source. This information can also better guide monitoring as the smaller sources that may not require full remediation can be located. Surveys can then be flown over various time periods to track any movement or changes to these sources.

Similarly, for nuclear emergency response the UAS would aid responders by providing more detailed information on the source locations. Responders may need to deal with multiple sources of similar or varying strength within an area, and will need to be able to locate all sources to guide evacuation plans and movements of security personnel. 
This research provides preliminary results from the use of a directional radiometric UAS. Future work could be done to examine the errors involved with the processing methods used. An error analysis could be used to determine how the methods can be improved. For example, a more accurate GNSS system could be used to improve positioning, a laser altimeter could be used to improve altitude above ground level measurements, or a different crystal configuration could be used to improve direction reconstruction results along the horizon.

Additional work could also be completed to integrate the autopilot and ARDUO data streams in real time. This could reduce the time taken in post-processing, as well as provide the potential for the UAS to produce maps while it is surveying. The real time integration of the direction information could also be used to guide the UAS. This can reduce the need to perform a grid search for a radioactive source, but rather use the limited flight time of the UAS to fly directly towards the area where the source is located. This could be beneficial for both environmental and security operations by being able to perform a more targeted search for radioactive sources and reduce the time taken to perform these searches. 


\section{Bibliography}

[1] C. M. Chen, L. E. Sinclair, R. Fortin, M. Coyle, and C. Samson, "Flight performance of the advanced radiation detector for UAV operations (ARDUO)," Orally presented at the 17th Symposium on radiation measurements and applications, Ann Arbor, MI, 11-14 June, 2018.

[2] _ _ , "In-flight performance of the Advanced Radiation Detector for UAV Operations (ARDUO)," Nuclear Instruments and Methods in Physics Research Section A: Accelerators, Spectrometers, Detectors and Associated Equipment, 2018.

[3] ING Robotic Aviation Inc., 2018, 1455 Youville Drive, Orléans, ON K1C $6 Z 7$.

[4] Scionix Holland, 2018, regulierenring 5, 3981 LA Bunnik, The Netherlands.

[5] Radiation Solutions Inc., 2018, 5875 Whittle Rd, Mississauga, ON L4Z 2H4.

[6] International Atomic Energy Agency, Airborne Gamma Ray Spectrometer Surveying, ser. Technical Reports Series. Vienna: INTERNATIONAL ATOMIC ENERGY AGENCY, 1991, no. 323. [Online]. Available: http://www-pub.iaea. org/books/IAEABooks/1427/Airborne-Gamma-Ray-Spectrometer-Surveying

[7] —_, Guidelines for Radioelement Mapping Using Gamma Ray Spectrometry Data. Vienna: International Atomic Energy Agency, 2003, iAEA-TECDOC1363. 
[8] ING Aviation Robotics Inc., Responder Operator Handbook V.1.6.1, 1455 Youville Drive, Unit 112, Ottawa, Ontario, Canada, K1C 6Z7, December 2016.

[9] Esri, "How kernel density works," 2018, accessed 28 June. [Online]. Available: http://web.archive.org/web/20080207010024/http://www.808multimedia. com/winnt/kernel.htm

[10] B. Beckman, A.-R. Green, L. E. Sinclair, B. Fairbrother, and D. White, "Radiological dispersal field trial involving unmanned air and ground systems," 2018, manuscript in preparation.

[11] ArduPilot Dev Team, "Mission planner," 2016, version: 1.3.48.

[12] P. G. Martin, O. D. Payton, J. S. Fardoulis, D. A. Richards, Y. Yamashiki, and T. B. Scott, "Low altitude unmanned aerial vehicle for characterising remediation effectiveness following the fdnpp accident," Journal of Environmental Radioactivity, vol. 151, pp. 58 - 63, 2016.

[13] J. Towler, B. Krawiec, and K. Kochersberger, "Radiation mapping in postdisaster environments using an autonomous helicopter," Remote Sensing, vol. 4, no. 7, pp. 1995-2015, 2012.

[14] B. Li, Y. Zhu, Z. Wang, C. Li, Z.-R. Peng, and L. Ge, "Use of multi-rotor unmanned aerial vehicles for radioactive source search," Remote Sensing, vol. 10, no. 5,2018 .

[15] R. Pöllänen, H. Toivonen, K. Peräjärvi, T. Karhunen, T. Ilander, J. Lehtinen, K. Rintala, T. Katajainen, J. Niemelä, and M. Juusela, "Radiation surveillance using an unmanned aerial vehicle," Applied Radiation and Isotopes, vol. 67, no. 2, pp. $340-344,2009$. 
[16] Y. Sanada and T. Torii, "Aerial radiation monitoring around the fukushima dai-ichi nuclear power plant using an unmanned helicopter," Journal of Environmental Radioactivity, vol. 139, pp. 294 - 299, 2015.

[17] K. Boudergui, F. Carrel, T. Domenech, N. Guénard, J. P. Poli, A. Ravet, V. Schoepff, and R. Woo, "Development of a drone equipped with optimized sensors for nuclear and radiological risk characterization," in 2011 2nd International Conference on Advancements in Nuclear Instrumentation, Measurement Methods and their Applications, June 2011, pp. 1-9.

[18] K. Kurvinen, P. Smolander, R. Pöllänen, S. Kuukankorpi, M. Kettunen, and J. Lyytinen, "Design of a radiation surveillance unit for an unmanned aerial vehicle," Journal of Environmental Radioactivity, vol. 81, no. 1, pp. 1 - 10, 2005.

[19] K. Kochersberger, K. Kroeger, B. Krawiec, E. Brewer, and T. Weber, "Postdisaster remote sensing and sampling via an autonomous helicopter," Journal of Field Robotics, vol. 31, no. 4, pp. 510-521, 2014.

[20] J. W. MacFarlane, O. D. Payton, A. C. Keatley, G. P. T. Scott, H. Pullin, R. A. Crane, M. Smilion, I. Popescu, V. Curlea, and T. B. Scott, "Lightweight aerial vehicles for monitoring, assessment and mapping of radiation anomalies," Journal of Environmental Radioactivity, vol. 136, pp. 127 - 130, 2014.

[21] P. Martin, O. Payton, J. Fardoulis, D. Richards, and T. Scott, "The use of unmanned aerial systems for the mapping of legacy uranium mines," Journal of Environmental Radioactivity, vol. 143, pp. 135 - 140, 2015.

[22] P. Killeen, C. Mwenifumbo, and K. Ford, "11.14 - tools and techniques: Radiometric methods," in Treatise on Geophysics (Second Edition), second edition ed., G. Schubert, Ed. Oxford: Elsevier, 2015, pp. 447 - 
524. [Online]. Available: http://www.sciencedirect.com/science/article/pii/ B9780444538024002098

[23] Y. Sanada, T. Sugita, Y. Nishizawa, A. Kondo, and T. Torii, "The aerial radiation monitoring in japan after the fukushima daiichi nuclear power plant accident," Progress in Nuclear Science and Technology, vol. 4, no. 7, 2014.

[24] G. F. Knoll, Radiation Detection and Measurement; 3rd ed. New York, NY: Wiley, 2000.

[25] G. Nelson and D. Reilly, "Gamma-ray interactions with matter," Tech. Rep., 3 1991.

[26] Natural Resources Canada, "Cagdb - natgam - allan lake carbonatite (brent crater)," 1985, accessed 10 September. [Online]. Available: https: //open.canada.ca/data/en/dataset/6b0062dc-4c78-543a-8ae8-f671488ec7f2

[27] European Space Agency, "Sentinel-2A multispectral instrument data," 2016, datatake GS2A_20160924T161342_N02.04.

[28] R. Fortin, J. R. Harvey, C. M. Chen, M. J. Coyle, L. E. Sinclair, and M. B. McClenaghan, "Surveying a glacial dispersal train with an unmanned aerial vehicle: A case study at the Allan Lake carbonatite," Poster presented at CANQUA/AMQUA 2018: Joint meeting of the Canadian and Americal Quaternary Associations, Ottawa, Ontario, Canada, 07-11 August, 2018.

[29] Transport Canada, "Canadian aviation regulations: Exemption from sections 602.41 and 603.66 of the canadian aviation regulations," 2016, retrieved from http://www.tc.gc.ca/civilaviation/regserv/affairs/exemptions/docs/en/2879.htm.

[30] Radiation Solutions Inc., "Rs-500 advanced digital gamma-ray spectrometer for airborne geophysical exploration and geological mapping," 2016, accessed 
27 August. [Online]. Available: http://www.radiationsolutions.ca/wp-content/ uploads/2016/06/RS500.pdf

[31] PX4 Dev Team, modified by ING Robotic Aviation Inc., "Pixhawk 1 flight controller," 2018, firmware Version: 3.3.3-ING03. [Online]. Available: https://docs.px4.io/en/flight_controller/pixhawk.html

[32] J. Gundlach, Designing Unmanned Aircraft Systems: A Comprehensive Approach, 2nd ed. Reston, Virginia: American Institute of Aeronautics and Astronautics Inc., 2014.

[33] ArduPilot Dev Team, "Extended kalman filter navigation overview and tuning," 2016, accessed 10 September. [Online]. Available: http://ardupilot.org/dev/ docs/extended-kalman-filter.html

[34] I. Kawrakow, E. Mainegra-Hing, D. W. O. Rogers, F. Tessier, and B. R. B. Walters, "The EGSnrc code system: Monte Carlo simulation of electron and photon transport," National Research Council Canada, Tech. Rep. PIRS-701, 2018. [Online]. Available: http://nrc-cnrc.github.io/EGSnrc/doc/ pirs701-egsnrc.pdf

[35] L. E. Sinclair, R. Fortin, M. J. Coyle, R. A. Van Brabant, and A. McCann, "Advanced radiation detector for unmanned aerial vehicle operations (ARDUO)," Poster presented at IEEE Symposium on Radiation Measurements and Applications, Berkeley, California, United States, 22 - 26 May, 2016.

[36] J. Hubbell and S. Seltzer, "Tables of x-ray mass attenuation coefficients and mass energy-absorption coefficients (version 1.4)," Tech. Rep., 2004, https://dx.doi.org/10.18434/T4D01F.

[37] W. Robertson, "Draft specifications for 5 sets of orthogonal square field nulling coils," December 1965. 
[38] L. E. Sinclair, "Proposed design for directional detector for uav," December, 2015, Natural Resources Canada internal report.

[39] Transport Canada, "Exemption from Sections 602.41 and 603.66 of the Canadian Aviation Regulations," 22 December 2016, http://www.tc.gc.ca/civilaviation/regserv/affairs/exemptions/docs/en/2879.htm. Accessed: 20 August 2018.

[40] C. F. F. Karney, "Geographiclib library," https://geographiclib.sourceforge.io/ 1.38/, 2014, version: 1.38-5.10.

[41] Esri, "How idw works," 2018, accessed 28 June. [Online]. Available: http://desktop.arcgis.com/en/arcmap/10.3/tools/3d-analyst-toolbox/ how-idw-works.htm

[42] N. Gadd, Surficial geology of Ottawa map-area, Ontario and Quebec. Geological Survey of Canada, 1963, no. 62-16.

[43] Google Earth, "Imagery: Google," 2016.

[44] —_, "Imagery: Digitalglobe," 2018.

[45] V. Carlson, "Bedrock topography, Medicine Hat area; Res. Coun. alberta map," 1970, scale 1:250,000.

[46] R. Heath, "Scintillation spectrometry. gamma-ray spectrum catalogue. 2nd edition. volume 1 of $2 . "$

[47] L. E. Sinclair and R. Fortin, "Spatial deconvolution of aerial radiometric survey and its application to the fallout from a radiological dispersal device," Journal of Environmental Radioactivity, vol. 197, pp. 39 - 47, 2019. 
[48] F. A. Marshall, "Reconstruction of the spatial distribution of surface activity concentration for an in-situ, gamma-ray, truck-borne survey," Master's thesis, Carleton University, 2014. 